\title{
From quarks to nucleons in dark matter direct detection
}

\author{
Fady Bishara, ${ }^{a}$ Joachim Brod, ${ }^{b}$ Benjamin Grinstein ${ }^{c}$ and Jure Zupan ${ }^{d, e}$ \\ ${ }^{a}$ Rudolf Peierls Centre for Theoretical Physics, University of Oxford, \\ OX1 3NP Oxford, U.K. \\ ${ }^{b}$ Fakultät für Physik, Technische Universität Dortmund, \\ D-44221 Dortmund, Germany \\ ${ }^{c}$ Department of Physics, University of California-San Diego, \\ La Jolla, CA 92093, U.S.A. \\ ${ }^{d}$ Department of Physics, University of Cincinnati, \\ Cincinnati, Ohio 45221, U.S.A. \\ e Theory Division, CERN, \\ CH-1211 Geneva 23, Switzerland \\ E-mail: fady.bishara@physics.ox.ac.uk, joachim.brod@tu-dortmund.de, \\ bgrinstein@ucsd.edu, zupanje@ucmail.uc.edu
}

ABSTRACT: We provide expressions for the nonperturbative matching of the effective field theory describing dark matter interactions with quarks and gluons to the effective theory of nonrelativistic dark matter interacting with nonrelativistic nucleons. We give expressions of leading and subleading order in chiral counting. In general, a single partonic operator matches onto several nonrelativistic operators already at leading order in chiral counting. Keeping only one operator at the time in the nonrelativistic effective theory thus does not properly describe the scattering in direct detection. The matching of the axial-axial partonic level operator, as well as the matching of the operators coupling DM to the QCD anomaly term, include naively momentum suppressed terms. However, these are still of leading chiral order due to pion poles and can be numerically important.

KEywords: Beyond Standard Model, Chiral Lagrangians

ARXIV EPRINT: 1707.06998 


\section{Contents}

1 Introduction 1

2 Fermionic dark matter 5

$\begin{array}{ll}2.1 \text { Leading-order expressions } & 7\end{array}$

$\begin{array}{lll}2.2 & \text { Subleading corrections } & 9\end{array}$

3 Scalar dark matter $\quad 11$

4 Examples 12

5 Conclusions $\quad 18$

A Values of the nucleon form factors $\quad 20$

A.1 Vector current 20

A.2 Axial vector current 21

A.3 Scalar current 23

A.4 Pseudoscalar current 24

A.5 CP-even gluonic current $\quad 25$

A.6 CP-odd gluonic current 25

A.7 Tensor current 26

B Nonrelativistic expansion of currents for fermions 28

C NLO expressions for fermionic DM 31

D Nonrelativistic expansion for scalar DM 33

E The expressions for the non-relativistic coefficients 35

\section{Introduction}

Dark Matter (DM) direct detection, where DM scatters on a target nucleus, is well described by Effective Field Theory (EFT) [1-18], which is essential to compare results of different direct detection experiments [19]. The maximal momentum exchange between $\mathrm{DM}$ and the nucleus is $q_{\max } \lesssim 200 \mathrm{MeV}$, see figure 1 . This means that one is able to use chiral counting, with an expansion parameter $q / \Lambda_{\text {ChEFT }} \lesssim 0.3$ to organize different contributions in the nucleon EFT for each of the operators coupling DM to quarks and gluons $[1,16,20-24]$. In this paper we rewrite the leading-order (LO) results in the chiral expansion of ref. [1] in terms of single-nucleon form factors. We also extend these results to 

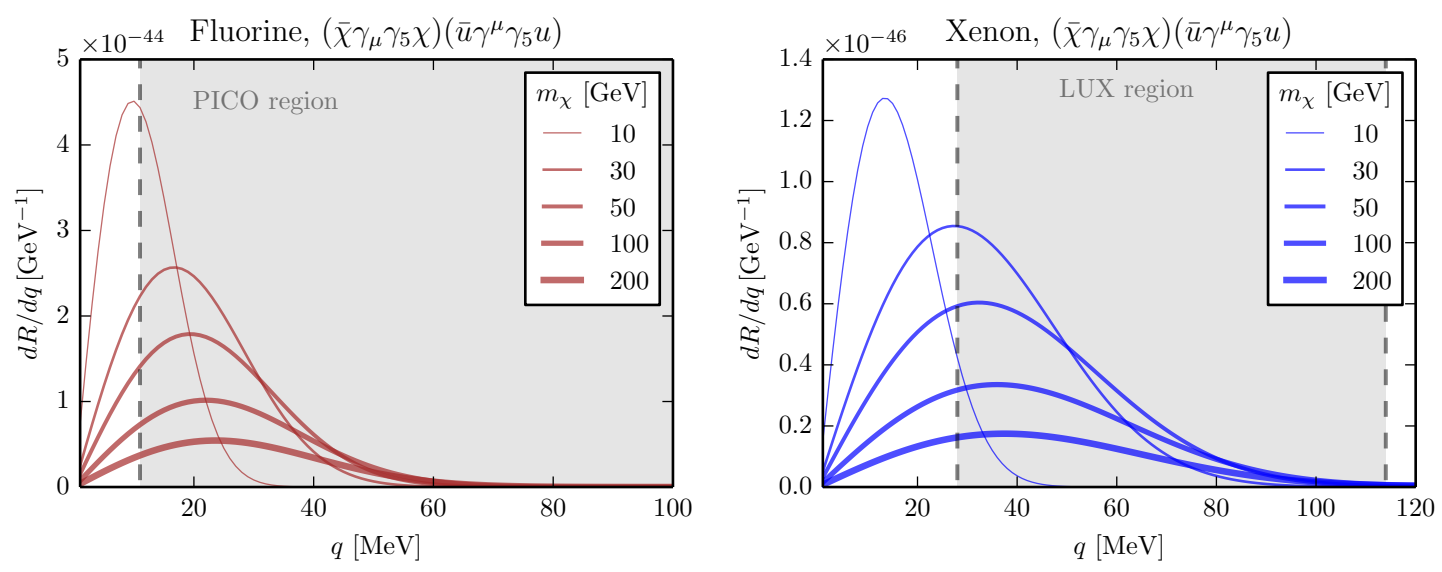

Figure 1. The momentum exchange distributions for DM scattering on a representative light nucleus, ${ }^{19} \mathrm{~F}$, (left) and heavy nucleus, Xe, (right) for spin-dependent scattering. The Wilson coefficient of the operator is set to $(1 \mathrm{TeV})^{-2}$ in both cases and we summed the contributions of the xenon isotopes weighted by their natural abundances. The curves of different thicknesses correspond to different dark matter masses in $\mathrm{GeV}$ as shown in the plot legends. The approximate experimental thresholds are denoted by dashed vertical lines. For fluorine, we use the PICO threshold region $E_{R}>3.3 \mathrm{keV}$ [28] while for LUX, we use the approximate region $E_{R} \in[3,50] \mathrm{keV}$ [29].

higher orders in the $\left(q / \Lambda_{\mathrm{ChEFT}}\right)^{2}$ expansion up to the order where two-nucleon currents are expected to become important (for the discussion of two-nucleon currents and numerical estimates see $[16-18,25,26])$. We give several numerical examples illustrating that it is not always justified to use momentum-independent coefficients in the nonrelativistic EFT for DM interactions with nucleons [3-5]. One needs to include the light-meson poles when DM couples to axial quark current or to the QCD anomaly term, to capture the leading effects of the strong interactions. Similarly, assuming that only one of the norelativistic EFT operators contributes may be equally hard to justify in a more complete UV theory. From a particle-physics point of view it is easier to interpret the results of DM direct detection experiments if one uses an EFT where DM couples to quark and gluons.

Our starting point is thus the interaction Lagrangian between DM and the SM quarks, gluons, and photon, given by a sum of higher dimension operators,

$$
\mathcal{L}_{\chi}=\sum_{a, d} \hat{\mathcal{C}}_{a}^{(d)} \mathcal{Q}_{a}^{(d)}, \quad \text { where } \quad \hat{\mathcal{C}}_{a}^{(d)}=\frac{\mathcal{C}_{a}^{(d)}}{\Lambda^{d-4}}
$$

Here the $\mathcal{C}_{a}^{(d)}$ are dimensionless Wilson coefficients, while $\Lambda$ can be identified with the mass of the mediators between DM and the SM (for couplings of order unity). The sums run over the dimensions of the operators, $d=5,6,7$ and the operator labels, $a$. Depending on the operator, the label ' $a$ ' either denotes an operator number or a number and a flavor index if the operator contains a SM fermion bilinear. We keep all the operators of dimensions five and six, and all the operators of dimension seven that couple DM to gluons. Among the dimension-seven operators that couple DM to quarks we exclude from the discussion the 
operators that are additionally suppressed by derivatives but have otherwise the same chiral structure as the dimension-six operators (for the treatment of these operators see [27]).

There are two dimension-five operators,

$$
\mathcal{Q}_{1}^{(5)}=\frac{e}{8 \pi^{2}}\left(\bar{\chi} \sigma^{\mu \nu} \chi\right) F_{\mu \nu}, \quad \mathcal{Q}_{2}^{(5)}=\frac{e}{8 \pi^{2}}\left(\bar{\chi} \sigma^{\mu \nu} i \gamma_{5} \chi\right) F_{\mu \nu}
$$

where $F_{\mu \nu}$ is the electromagnetic field strength tensor and $\chi$ is the DM field, assumed here to be a Dirac particle. The magnetic dipole operator $\mathcal{Q}_{1}^{(5)}$ is $\mathrm{CP}$ even, while the electric dipole operator $\mathcal{Q}_{2}^{(5)}$ is CP odd. The dimension-six operators are

$$
\begin{array}{ll}
\mathcal{Q}_{1, q}^{(6)}=\left(\bar{\chi} \gamma_{\mu} \chi\right)\left(\bar{q} \gamma^{\mu} q\right), & \mathcal{Q}_{2, q}^{(6)}=\left(\bar{\chi} \gamma_{\mu} \gamma_{5} \chi\right)\left(\bar{q} \gamma^{\mu} q\right), \\
\mathcal{Q}_{3, q}^{(6)}=\left(\bar{\chi} \gamma_{\mu} \chi\right)\left(\bar{q} \gamma^{\mu} \gamma_{5} q\right), & \mathcal{Q}_{4, q}^{(6)}=\left(\bar{\chi} \gamma_{\mu} \gamma_{5} \chi\right)\left(\bar{q} \gamma^{\mu} \gamma_{5} q\right),
\end{array}
$$

and we also include a subset of the dimension-seven operators, namely ${ }^{1}$

$$
\begin{aligned}
\mathcal{Q}_{1}^{(7)} & =\frac{\alpha_{s}}{12 \pi}(\bar{\chi} \chi) G^{a \mu \nu} G_{\mu \nu}^{a}, & \mathcal{Q}_{2}^{(7)} & =\frac{\alpha_{s}}{12 \pi}\left(\bar{\chi} i \gamma_{5} \chi\right) G^{a \mu \nu} G_{\mu \nu}^{a}, \\
\mathcal{Q}_{3}^{(7)} & =\frac{\alpha_{s}}{8 \pi}(\bar{\chi} \chi) G^{a \mu \nu} \widetilde{G}_{\mu \nu}^{a}, & \mathcal{Q}_{4}^{(7)} & =\frac{\alpha_{s}}{8 \pi}\left(\bar{\chi} i \gamma_{5} \chi\right) G^{a \mu \nu} \widetilde{G}_{\mu \nu}^{a}, \\
\mathcal{Q}_{5, q}^{(7)} & =m_{q}(\bar{\chi} \chi)(\bar{q} q), & \mathcal{Q}_{6, q}^{(7)} & =m_{q}\left(\bar{\chi} i \gamma_{5} \chi\right)(\bar{q} q), \\
\mathcal{Q}_{7, q}^{(7)} & =m_{q}(\bar{\chi} \chi)\left(\bar{q} i \gamma_{5} q\right), & \mathcal{Q}_{8, q}^{(7)} & =m_{q}\left(\bar{\chi} i \gamma_{5} \chi\right)\left(\bar{q} i \gamma_{5} q\right), \\
\mathcal{Q}_{9, q}^{(7)} & =m_{q}\left(\bar{\chi} \sigma^{\mu \nu} \chi\right)\left(\bar{q} \sigma_{\mu \nu} q\right), & \mathcal{Q}_{10, q}^{(7)} & =m_{q}\left(\bar{\chi} i \sigma^{\mu \nu} \gamma_{5} \chi\right)\left(\bar{q} \sigma_{\mu \nu} q\right) .
\end{aligned}
$$

Here $G_{\mu \nu}^{a}$ is the QCD field strength tensor, while $\widetilde{G}_{\mu \nu}=\frac{1}{2} \varepsilon_{\mu \nu \rho \sigma} G^{\rho \sigma}$ is its dual, and $a=$ $1, \ldots, 8$ are the adjoint color indices. Moreover, $q=u, d, s$ denote the light quarks (we limit ourselves to flavor conserving operators). Note that we include two more dimension-seven operators than in [1], so that we have all the operators included in [30]. The remaining dimension-seven operators coupling DM to quarks are listed in [27], while the effect of dimension-seven operators coupling DM to photons is discussed in [31]. There are also the leptonic equivalents of the operators $\mathcal{Q}_{1, q}^{(6)}, \ldots, \mathcal{Q}_{4, q}^{(6)}$, and $\mathcal{Q}_{5, q}^{(7)}, \ldots, \mathcal{Q}_{10, q}^{(7)}$, with $q \rightarrow \ell$.

The aim of this paper is to provide compact expressions for the non-perturbative matching at $\mu \simeq 2 \mathrm{GeV}$ between the EFT with three quark flavors, given by eq. (1.1), and the theory of DM interacting with nonrelativistic nucleons, given by

$$
\mathcal{L}_{\mathrm{NR}}=\sum_{i, N} c_{i}^{N}\left(q^{2}\right) \mathcal{O}_{i}^{N}
$$

For each operator the matching is done using the heavy baryon chiral perturbation theory expansion [32] up to the order for which the scattering amplitudes are still parametrically dominated by single-nucleon currents. The relevant Galilean-invariant operators with at

\footnotetext{
${ }^{1}$ Note that the definition of the operator $\mathcal{Q}_{8, q}^{(7)}$ differs by a sign from the definition used in [1].
} 


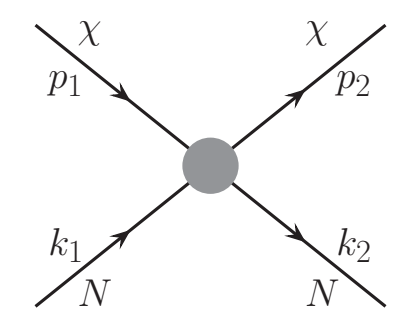

Figure 2. The kinematics of DM scattering on nucleons, $\chi\left(p_{1}\right) N\left(k_{1}\right) \rightarrow \chi\left(p_{2}\right) N\left(k_{2}\right)$.

most two derivatives are

$$
\begin{array}{rlrl}
\mathcal{O}_{1}^{N} & =\mathbb{1}_{\chi} \mathbb{1}_{N}, & \mathcal{O}_{2}^{N} & =\left(v_{\perp}\right)^{2} \mathbb{1}_{\chi} \mathbb{1}_{N}, \\
\mathcal{O}_{3}^{N} & =\mathbb{1}_{\chi} \vec{S}_{N} \cdot\left(\vec{v}_{\perp} \times \frac{i \vec{q}}{m_{N}}\right), & \mathcal{O}_{4}^{N} & =\vec{S}_{\chi} \cdot \vec{S}_{N}, \\
\mathcal{O}_{5}^{N} & =\vec{S}_{\chi} \cdot\left(\vec{v}_{\perp} \times \frac{i \vec{q}}{m_{N}}\right) \mathbb{1}_{N}, & \mathcal{O}_{6}^{N}=\left(\vec{S}_{\chi} \cdot \frac{\vec{q}}{m_{N}}\right)\left(\vec{S}_{N} \cdot \frac{\vec{q}}{m_{N}}\right), \\
\mathcal{O}_{7}^{N}=\mathbb{1}_{\chi}\left(\vec{S}_{N} \cdot \vec{v}_{\perp}\right), & \mathcal{O}_{8}^{N}=\left(\vec{S}_{\chi} \cdot \vec{v}_{\perp}\right) \mathbb{1}_{N}, \\
\mathcal{O}_{9}^{N}=\vec{S}_{\chi} \cdot\left(\frac{i \vec{q}}{m_{N}} \times \vec{S}_{N}\right), & \mathcal{O}_{10}^{N}=-\mathbb{1}_{\chi}\left(\vec{S}_{N} \cdot \frac{i \vec{q}}{m_{N}}\right), \\
\mathcal{O}_{11}^{N}=-\left(\vec{S}_{\chi} \cdot \frac{i \vec{q}}{m_{N}}\right) \mathbb{1}_{N}, & \mathcal{O}_{12}^{N}=\vec{S}_{\chi} \cdot\left(\vec{S}_{N} \times \vec{v}_{\perp}\right), \\
\mathcal{O}_{13}^{N}=-\left(\vec{S}_{\chi} \cdot \vec{v}_{\perp}\right)\left(\vec{S}_{N} \cdot \frac{i \vec{q}}{m_{N}}\right), & \mathcal{O}_{14}^{N}=-\left(\vec{S}_{\chi} \cdot \frac{i \vec{q}}{m_{N}}\right)\left(\vec{S}_{N} \cdot \vec{v}_{\perp}\right),
\end{array}
$$

and in addition

$$
\mathcal{O}_{2 b}^{N}=\left(\vec{S}_{N} \cdot \vec{v}_{\perp}\right)\left(\vec{S}_{\chi} \cdot \vec{v}_{\perp}\right)
$$

where $N=p, n$. At next-to-leading order (NLO) we also need one operator with three derivatives,

$$
\mathcal{O}_{15}^{N}=-\left(\vec{S}_{\chi} \cdot \frac{\vec{q}}{m_{N}}\right)\left(\left(\vec{S}_{N} \times \vec{v}_{\perp}\right) \cdot \frac{\vec{q}}{m_{N}}\right) .
$$

Our definition of momentum exchange differs from [5] by a minus sign, cf. figure 2,

$$
\vec{q}=\vec{k}_{2}-\vec{k}_{1}=\vec{p}_{1}-\vec{p}_{2}, \quad \vec{v}_{\perp}=\left(\vec{p}_{1}+\vec{p}_{2}\right) /\left(2 m_{\chi}\right)-\left(\vec{k}_{1}+\vec{k}_{2}\right) /\left(2 m_{N}\right),
$$

while the operators coincide with those defined in [5]. Each insertion of $\vec{q}$ is accompanied by a factor of $1 / m_{N}$, so that all of the above operators have the same dimensionality.

This paper is organized as follows: in section 2 we give the matching conditions for fermionic DM and in section 3 for scalar DM, while in section 4 we present several examples illustrating the importance of keeping all terms of the same chiral order. Section 5 contains our conclusions. The numerical values of the form factors are collected in appendix A, appendix $\mathrm{B}$ contains the nonrelativistic expansion of the fermionic DM and nucleon currents, appendix $\mathrm{C}$ the extended NLO operator basis, appendix D the NLO results for scalar DM, while appendix E gives the results for fermionic DM in terms of coefficients of the nonrelativistic operators. 


\section{$2 \quad$ Fermionic dark matter}

The hadronization of operators $\mathcal{Q}_{1, q}^{(6)}, \ldots, \mathcal{Q}_{10, q}^{(7)}$, in eqs. (1.3)-(1.9) leads at LO in the chiral expansion only to single-nucleon currents [1]. The scattering of DM on a nucleus with mass number $A$ is given by a sum of $A$-nucleon irreducible amplitudes with one DM current insertion. These amplitudes scale as $\mathcal{M}_{A, \chi} \sim\left(q / \Lambda_{\text {ChEFT }}\right)^{\nu}$ where the power counting exponent $\nu$ is given explicitly in [1]. This counting was first derived by Weinberg in [21] see also [16, 22]. In the case of our EFT basis, the matrix elements of the operators scale as $q^{\nu_{\mathrm{LO}}}$, with $[1,27]$

$$
\begin{array}{llll}
{\left[\mathcal{Q}_{1, q}^{(6)}\right]_{\mathrm{LO}} \sim 1,} & {\left[\mathcal{Q}_{2, q}^{(6)}\right]_{\mathrm{LO}} \sim q,} & {\left[\mathcal{Q}_{3, q}^{(6)}\right]_{\mathrm{LO}} \sim q,} & {\left[\mathcal{Q}_{4, q}^{(6)}\right]_{\mathrm{LO}} \sim 1,} \\
{\left[\mathcal{Q}_{1}^{(7)}\right]_{\mathrm{LO}} \sim 1,} & {\left[\mathcal{Q}_{2}^{(7)}\right]_{\mathrm{LO}} \sim q,} & {\left[\mathcal{Q}_{3}^{(7)}\right]_{\mathrm{LO}} \sim q,} & {\left[\mathcal{Q}_{4}^{(7)}\right]_{\mathrm{LO}} \sim q^{2},} \\
{\left[\mathcal{Q}_{5, q}^{(7)}\right]_{\mathrm{LO}} \sim q^{2},} & {\left[\mathcal{Q}_{6, q}^{(7)}\right]_{\mathrm{LO}} \sim q^{3},} & {\left[\mathcal{Q}_{7, q}^{(7)}\right]_{\mathrm{LO}} \sim q,} & {\left[\mathcal{Q}_{8, q}^{(7)}\right]_{\mathrm{LO}} \sim q^{2},} \\
{\left[\mathcal{Q}_{9, q}^{(7)}\right]_{\mathrm{LO}} \sim 1,} & {\left[\mathcal{Q}_{10, q}^{(7)}\right]_{\mathrm{LO}} \sim q,} & &
\end{array}
$$

counting $m_{q} \sim m_{\pi}^{2} \sim q^{2}$, and not displaying a common scaling factor. The LO contributions are either due to scattering of DM on a single nucleon (the first diagram in figure 3), or on a pion that attaches to the nucleon (the second diagram), or both. The contributions from DM scattering on two-nucleon currents arise at $\mathcal{O}\left(q^{\nu_{\mathrm{LO}}+1}\right)$ for $\mathcal{O}_{2, q}^{(6)}, \mathcal{O}_{5, q}^{(7)}$, and $\mathcal{O}_{6, q}^{(7)}$, at $\mathcal{O}\left(q^{\nu_{\mathrm{LO}}+2}\right)$ for $\mathcal{O}_{1, q}^{(6)}$, and at $\mathcal{O}\left(q^{\nu_{\mathrm{LO}}+3}\right)$ for all the other operators. Up to these orders, the hadronization of the operators $\mathcal{Q}_{1, q}^{(6)}, \ldots, \mathcal{Q}_{10, q}^{(7)}$ can thus be described by using form factors for single-nucleon currents.

The form factors are given by

$$
\begin{aligned}
\left\langle N^{\prime}\left|\bar{q} \gamma^{\mu} q\right| N\right\rangle & =\bar{u}_{N}^{\prime}\left[F_{1}^{q / N}\left(q^{2}\right) \gamma^{\mu}+\frac{i}{2 m_{N}} F_{2}^{q / N}\left(q^{2}\right) \sigma^{\mu \nu} q_{\nu}\right] u_{N}, \\
\left\langle N^{\prime}\left|\bar{q} \gamma^{\mu} \gamma_{5} q\right| N\right\rangle & =\bar{u}_{N}^{\prime}\left[F_{A}^{q / N}\left(q^{2}\right) \gamma^{\mu} \gamma_{5}+\frac{1}{2 m_{N}} F_{P^{\prime}}^{q / N}\left(q^{2}\right) \gamma_{5} q^{\mu}\right] u_{N}, \\
\left\langle N^{\prime}\left|m_{q} \bar{q} q\right| N\right\rangle & =F_{S}^{q / N}\left(q^{2}\right) \bar{u}_{N}^{\prime} u_{N}, \\
\left\langle N^{\prime}\left|m_{q} \bar{q} i \gamma_{5} q\right| N\right\rangle & =F_{P}^{q / N}\left(q^{2}\right) \bar{u}_{N}^{\prime} i \gamma_{5} u_{N}, \\
\left\langle N^{\prime}\left|\frac{\alpha_{s}}{12 \pi} G^{a \mu \nu} G_{\mu \nu}^{a}\right| N\right\rangle & =F_{G}^{N}\left(q^{2}\right) \bar{u}_{N}^{\prime} u_{N}, \\
\left\langle N^{\prime}\left|\frac{\alpha_{s}}{8 \pi} G^{a \mu \nu} \tilde{G}_{\mu \nu}^{a}\right| N\right\rangle & =F_{\tilde{G}}^{N}\left(q^{2}\right) \bar{u}_{N}^{\prime} i \gamma_{5} u_{N}, \\
\left\langle N^{\prime}\left|m_{q} \bar{q} \sigma^{\mu \nu} q\right| N\right\rangle & =\bar{u}_{N}^{\prime}\left[F_{T, 0}^{q / N}\left(q^{2}\right) \sigma^{\mu \nu}+\frac{i}{2 m_{N}} \gamma^{[\mu} q^{\nu]} F_{T, 1}^{q / N}\left(q^{2}\right)\right. \\
& \left.+\frac{i}{m_{N}^{2}} q^{[\mu} k_{12}^{\nu]} F_{T, 2}^{q / N}\left(q^{2}\right)\right] u_{N},
\end{aligned}
$$

where we have suppressed the dependence of nucleon states on their momenta, i.e. $\left\langle N^{\prime}\right| \equiv$ $\left\langle N\left(k_{2}\right)|| N,\right\rangle \equiv\left|N\left(k_{1}\right)\right\rangle$, and similarly, $\bar{u}_{N}^{\prime} \equiv \bar{u}_{N}\left(k_{2}\right), u_{N} \equiv u_{N}\left(k_{1}\right)$. The momentum exchange is $q^{\mu}=k_{2}^{\mu}-k_{1}^{\mu}$, while $k_{12}^{\mu}=k_{1}^{\mu}+k_{2}^{\mu}$. The form factors $F_{i}$ are functions of $q^{2}$ only.

The axial current, the pseudoscalar current, and the CP-odd gluonic current receive contributions from light pseudoscalar meson exchanges corresponding to the second dia- 

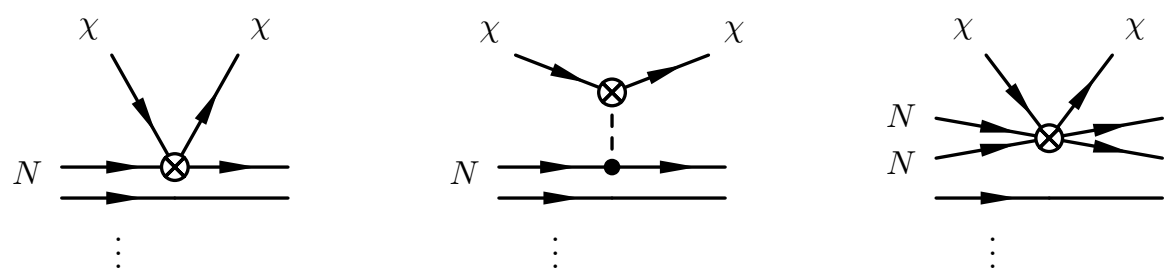

Figure 3. The chirally leading diagrams for DM-nucleus scattering (the first and second diagrams), and a representative diagram for two-nucleon scattering (the third diagram). The effective DMnucleon and DM-meson interactions are denoted by a circle, the dashed line denotes a pion, and the dots represent the remaining $A-2$ nucleon lines.

gram in figure 3. For small momenta exchanges, $q \sim m_{\pi}$, one can expand the form factors in $q^{2}$, as

$$
\begin{aligned}
F_{i}^{q / N}\left(q^{2}\right) & =\overbrace{\frac{m_{N}^{2}}{m_{\pi}^{2}-q^{2}} a_{i, \pi}^{q / N}+\frac{m_{N}^{2}}{m_{\eta}^{2}-q^{2}} a_{i, \eta}^{q / N}}^{\mathrm{LO}}+\overbrace{b_{i}^{q / N}}^{\mathrm{NLO}}+\cdots, \quad i=P, P^{\prime}, \\
F_{\tilde{G}}^{N}\left(q^{2}\right) & =\underbrace{\frac{q^{2}}{m_{\pi}^{2}-q^{2}} a_{\tilde{G}, \pi}^{N}+\frac{q^{2}}{m_{\eta}^{2}-q^{2}} a_{\tilde{G}, \eta}^{N}+b_{\tilde{G}}^{N}}_{\mathrm{LO}}+\underbrace{c_{\tilde{G}}^{N} q^{2}}_{\text {NLO }}+\cdots,
\end{aligned}
$$

where we kept both the pion and eta poles and denoted the order of the various terms in chiral counting. The coefficients $a_{i}, b_{i}, c_{i}$ are momentum-independent constants. Note that the pion and eta poles for the $G \tilde{G}$ operator are suppressed by $q^{2}$ and are thus of the same chiral order as the constant term, $b_{\tilde{G}}^{N}$. All the other form factors do not have a light pseudoscalar pole and can be Taylor expanded ${ }^{2}$ around $q^{2}=0$,

$$
F_{i}^{q / N}\left(q^{2}\right)=\underbrace{F_{i}^{q / N}(0)}_{\mathrm{LO}}+\underbrace{F_{i}^{\prime q / N}(0) q^{2}}_{\mathrm{NLO}}+\cdots,
$$

where the prime on $F$ denotes a derivative with respect to $q^{2}$. The values of $F_{i}^{q / N}(0)$, $F_{i}^{\prime q / N}(0)$, and $a_{i}, b_{i}, c_{i}$ are collected in appendix A.

The size of the form factors that do not have light-meson poles are, at zero recoil,

$$
\begin{aligned}
F_{1,2}^{q / N}(0), F_{A}^{q / N}(0) & \sim \mathcal{O}(1), & F_{1,2}^{s / N}(0), F_{A}^{s / N}(0) & \sim \mathcal{O}(0-0.05), \\
F_{S}^{q / N}(0) & \sim \mathcal{O}(0.03) m_{N}, & F_{S}^{s / N}(0) & \sim \mathcal{O}(0.05) m_{N}, \\
F_{G}^{N}(0) & \sim \mathcal{O}(0.1) m_{N}, & & \\
F_{T, 0 ; T, 1 ; T, 2}^{q / N}(0) & \sim \mathcal{O}(1) m_{q}, & F_{T, 0 ; T, 1 ; T, 2}^{s / N}(0) & \lesssim \mathcal{O}(0.001-0.2) m_{s} .
\end{aligned}
$$

(only here and in the remainder of the subsection we use the abbreviation $q=u, d$ ). The $s$-quark form factors are much smaller, with the exception of the scalar form factor.

\footnotetext{
${ }^{2}$ We assume that the NLO terms involving chiral logarithms of the form $\left(m_{\pi}^{2}-q^{2}\right) \log \left(m_{\pi}^{2}-q^{2}\right)$ were also expanded in $q^{2}$. This may give an effective expansion parameter $q^{2} /\left(\Lambda_{\mathrm{EFT}}\right)^{2}$ with $\Lambda_{\mathrm{EFT}}$ between $m_{\pi}$ and $4 \pi f$; however, numerically it is found to be closer to the latter, see appendix A.
} 
Their derivatives at zero recoil, which enter the NLO expressions, have a typical size $F_{i}^{\prime}(0) / F_{i}(0) \sim \mathcal{O}\left(1 / m_{N}^{2}\right)$, so that the corresponding corrections are expected at the level of several percent.

The coefficients of the terms in the form factors that contain the pion and eta poles, eqs. (2.9), (2.10), are approximately of the size

$$
\begin{aligned}
& a_{P^{\prime}, \pi}^{q / N}, a_{P^{\prime}, \eta}^{q / N} \sim \mathcal{O}(1), \quad a_{P^{\prime}, \pi}^{s / N}=0, \quad a_{P^{\prime}, \eta}^{s / N} \sim \mathcal{O}(1), \\
& a_{P, \pi}^{q / N}, a_{P, \eta}^{q / N} \sim \mathcal{O}(1) m_{q}, \quad a_{P, \pi}^{s / N}=0, \quad a_{P, \eta}^{s / N} \sim \mathcal{O}(1) m_{s}, \\
& a_{\tilde{G}, \pi}^{N}, a_{\tilde{G}, \eta}^{N}, b_{\tilde{G}}^{N} \sim \mathcal{O}(1) m_{N}
\end{aligned}
$$

\subsection{Leading-order expressions}

We first give the expressions for the nonrelativistic EFT Lagrangian (1.10) at LO in chiral counting. In this case we only need the values of $a_{i}^{\pi}, a_{i}^{\eta}, b_{\tilde{G}}^{N}$, and $F_{i}(0)$. In addition to taking the hadronic matrix elements of the quark and gluon currents we also take the nonrelativistic limit of both the DM currents and the nucleon currents. The expressions for this last step are collected in appendix B. The chirally leading hadronization of the dimension-five operators is thus given by

$$
\begin{aligned}
\mathcal{Q}_{1}^{(5)} & \rightarrow-\frac{\alpha}{2 \pi} F_{1}^{N}\left(\frac{1}{m_{\chi}} \mathcal{O}_{1}^{N}-4 \frac{m_{N}}{\vec{q}^{2}} \mathcal{O}_{5}^{N}\right)-\frac{2 \alpha}{\pi} \frac{\mu_{N}}{m_{N}}\left(\mathcal{O}_{4}^{N}-\frac{m_{N}^{2}}{\vec{q}^{2}} \mathcal{O}_{6}^{N}\right)+\mathcal{O}\left(q^{2}\right), \\
\mathcal{Q}_{2}^{(5)} & \rightarrow \frac{2 \alpha}{\pi} \frac{m_{N}}{\vec{q}^{2}} F_{1}^{N} \mathcal{O}_{11}^{N}+\mathcal{O}\left(q^{2}\right)
\end{aligned}
$$

with $F_{1}^{N}(0)=\delta_{p N}$ the nucleon charge, and $\mu_{N}$ the nucleon magnetic moment (see also appendix A.1). The dimension-six operators hadronize as

$$
\begin{aligned}
& \mathcal{Q}_{1, q}^{(6)} \rightarrow F_{1}^{q / N} \mathcal{O}_{1}^{N}+\mathcal{O}\left(q^{2}\right) \\
& \mathcal{Q}_{2, q}^{(6)} \rightarrow 2 F_{1}^{q / N} \mathcal{O}_{8}^{N}+2\left(F_{1}^{q / N}+F_{2}^{q / N}\right) \mathcal{O}_{9}^{N}+\mathcal{O}\left(q^{2}\right) \\
& \mathcal{Q}_{3, q}^{(6)} \rightarrow-2 F_{A}^{q / N}\left(\mathcal{O}_{7}^{N}-\frac{m_{N}}{m_{\chi}} \mathcal{O}_{9}^{N}\right)+\mathcal{O}\left(q^{2}\right) \\
& \mathcal{Q}_{4, q}^{(6)} \rightarrow-4 F_{A}^{q / N} \mathcal{O}_{4}^{N}+F_{P^{\prime}}^{q / N} \mathcal{O}_{6}^{N}+\mathcal{O}\left(q^{2}\right)
\end{aligned}
$$

while the hadronization of the gluonic dimension-seven operators is given by

$$
\begin{aligned}
& \mathcal{Q}_{1}^{(7)} \rightarrow F_{G}^{N} \mathcal{O}_{1}^{N}+\mathcal{O}\left(q^{2}\right) \\
& \mathcal{Q}_{2}^{(7)} \rightarrow-\frac{m_{N}}{m_{\chi}} F_{G}^{N} \mathcal{O}_{11}^{N}+\mathcal{O}\left(q^{3}\right) \\
& \mathcal{Q}_{3}^{(7)} \rightarrow F_{\tilde{G}}^{N} \mathcal{O}_{10}^{N}+\mathcal{O}\left(q^{3}\right) \\
& \mathcal{Q}_{4}^{(7)} \rightarrow \frac{m_{N}}{m_{\chi}} F_{\tilde{G}}^{N} \mathcal{O}_{6}^{N}+\mathcal{O}\left(q^{4}\right) .
\end{aligned}
$$


The hadronization of the dimension-seven operators with quark scalar currents results in

$$
\begin{aligned}
\mathcal{Q}_{5, q}^{(7)} & \rightarrow F_{S}^{q / N} \mathcal{O}_{1}^{N}+\mathcal{O}(q), \\
\mathcal{Q}_{6, q}^{(7)} & \rightarrow-\frac{m_{N}}{m_{\chi}} F_{S}^{q / N} \mathcal{O}_{11}^{N}+\mathcal{O}\left(q^{2}\right), \\
\mathcal{Q}_{7, q}^{(7)} & \rightarrow F_{P}^{q / N} \mathcal{O}_{10}^{N}+\mathcal{O}\left(q^{3}\right), \\
\mathcal{Q}_{8, q}^{(7)} & \rightarrow \frac{m_{N}}{m_{\chi}} F_{P}^{q / N} \mathcal{O}_{6}^{N}+\mathcal{O}\left(q^{4}\right),
\end{aligned}
$$

and for the tensor operators

$$
\begin{aligned}
\mathcal{Q}_{9, q}^{(7)} & \rightarrow 8 F_{T, 0}^{q / N} \mathcal{O}_{4}^{N}+\mathcal{O}\left(q^{2}\right) \\
\mathcal{Q}_{10, q}^{(7)} & \rightarrow-2 \frac{m_{N}}{m_{\chi}} F_{T, 0}^{q / N} \mathcal{O}_{10}^{N}+2\left(F_{T, 0}^{q / N}-F_{T, 1}^{q / N}\right) \mathcal{O}_{11}^{N}-8 F_{T, 0}^{q / N} \mathcal{O}_{12}^{N}+\mathcal{O}\left(q^{3}\right) .
\end{aligned}
$$

The nonrelativistic operators have been defined in (1.11)-(1.17). In the above expressions all the form factors $F_{i}^{q / N}$ are evaluated at $q^{2}=0$, apart from $F_{P, P^{\prime}}^{q / N}$ and $F_{\tilde{G}}^{N}$, where one needs to keep the two meson-pole terms in (2.9) and the first three terms in (2.10). The corresponding values of coefficients $c_{i}^{N}$ in the nonrelativistic Larangian, eq. (1.10), are given in appendix E.

Several comments are in order. First of all, in several cases a single operator describing the DM interactions with quarks and gluons matches onto more than one nonrelativistic operator in eqs. (1.11)-(1.19) already at leading chiral order. This occurs for

$$
\begin{aligned}
& \mathcal{Q}_{1}^{(5)}=\frac{e}{8 \pi^{2}}\left(\bar{\chi} \sigma^{\mu \nu} \chi\right) F_{\mu \nu} \sim Q_{p} \mathbb{1}_{\chi} \mathbb{1}_{N} / m_{\chi}+Q_{p} \vec{S}_{\chi} \cdot\left(\vec{v}_{\perp} \times i \vec{q}\right) \mathbb{1}_{N} / \vec{q}^{2} \\
&+\mu_{N} \vec{S}_{\chi} \cdot \vec{S}_{N} / m_{N}+\mu_{N}\left(\vec{S}_{\chi} \cdot \vec{q}\right)\left(\vec{S}_{N} \cdot \vec{q}\right) /\left(m_{N} \vec{q}^{2}\right) \\
& \mathcal{Q}_{2, q}^{(6)}=\left(\bar{\chi} \gamma_{\mu} \gamma_{5} \chi\right)\left(\bar{q} \gamma^{\mu} q\right) \sim\left(\vec{S}_{\chi} \cdot \vec{v}_{\perp}\right) \mathbb{1}_{N}+F_{1,2}^{q / N}(0) \vec{S}_{\chi} \cdot\left(i \vec{q} \times \vec{S}_{N}\right) / m_{N} \\
& \mathcal{Q}_{3, q}^{(6)}=\left(\bar{\chi} \gamma_{\mu} \chi\right)\left(\bar{q} \gamma^{\mu} \gamma_{5} q\right) \sim \Delta q_{N}\left[\mathbb{1}_{\chi}\left(\vec{S}_{N} \cdot \vec{v}_{\perp}\right)-\vec{S}_{\chi} \cdot\left(i \vec{q} \times \vec{S}_{N}\right) / m_{\chi}\right] \\
& \mathcal{Q}_{4, q}^{(6)}=\left(\bar{\chi} \gamma_{\mu} \gamma_{5} \chi\right)\left(\bar{q} \gamma^{\mu} \gamma_{5} q\right) \sim \Delta q_{N} \vec{S}_{\chi} \cdot \vec{S}_{N}+\frac{\Delta q_{N}}{m_{\pi}^{2}+\vec{q}^{2}}\left(\vec{S}_{\chi} \cdot \vec{q}\right)\left(\vec{S}_{N} \cdot \vec{q}\right) \\
& \mathcal{Q}_{10, q}^{(7)=} m_{q}\left(\bar{\chi} i \sigma^{\mu \nu} \gamma_{5} \chi\right)\left(\bar{q} \sigma_{\mu \nu} q\right) \sim \frac{m_{q}}{m_{\chi}} g_{T}^{q} \mathbb{1}_{\chi}\left(\vec{S}_{N} \cdot i \vec{q}\right)+\frac{m_{q}}{m_{N}}\left\{g_{T}^{q}, F_{T, 1}^{q / N}\right\}\left(\vec{S}_{\chi} \cdot i \vec{q}\right) \mathbb{1}_{N} \\
& \quad+m_{q} g_{T}^{q} \vec{S}_{\chi} \cdot\left(\vec{S}_{N} \times \vec{v}_{\perp}\right)
\end{aligned}
$$

where we only show the approximate dependence on the nonperturbative coefficients (here $Q_{p}=1$ is the proton charge, while the values of the axial charge $\Delta q_{N}$, the form factors $F_{1,2}^{q / N}(0)$ and the tensor charges, $\left.g_{T}^{q}, F_{T, 1}^{q / N}(0)\right)$ are given in appendix A.

The above results mean that it is not consistent within EFT to perform the direct detection analysis in the nonrelativistic basis and only turn on one of the operators $\mathcal{O}_{7}^{N}, \mathcal{O}_{8}^{N}, \mathcal{O}_{9}^{N}$ or $\mathcal{O}_{12}^{N}$, as they always come accompanied with other nonrelativistic operators, regardless of the UV operator that couples DM to quarks and gluons. On the other hand, the 
spin-independent operator $\mathcal{O}_{1}^{N}$ as well as the spin-dependent operator $\mathcal{O}_{4}^{N}$ can arise by themselves from $\mathcal{Q}_{1, q}^{(6)}, \mathcal{Q}_{1}^{(7)}, \mathcal{Q}_{5, q}^{q / N}$ and from $\mathcal{Q}_{9, q}^{(7)}$, respectively. Similarly, $\mathcal{O}_{6}^{N}, \mathcal{O}_{10}^{N}$, and $\mathcal{O}_{11}^{N}$ arise as the only leading operators in the nonrelativistic reduction of $\mathcal{Q}_{8, q}^{(7)}, \mathcal{Q}_{3}^{(7)}$ or $\mathcal{Q}_{7, q}^{(7)}$, and $\mathcal{Q}_{2}^{(7)}$ or $\mathcal{Q}_{6, q}^{(7)}$, respectively.

While it is true that the spin-dependent operator $\mathcal{O}_{4}^{N}$ can arise from the tensor-tensor operator $\mathcal{Q}_{9, q}^{(7)}$, this contribution would be of two-loop order in a perturbative UV theory of DM. The axial-axial operator $Q_{4, q}^{(6)}$, on the other hand, also leads to spin-dependent scattering and will arise at tree level. Therefore it will, if generated, typically dominate over $\mathcal{Q}_{9, q}^{(7)}$. The induced spin-dependent scattering arises from both the $\mathcal{O}_{4}^{N}=\vec{S}_{\chi} \cdot \vec{S}_{N}$ and $\mathcal{O}_{6}^{N}=\left(\vec{S}_{\chi} \cdot \vec{q}\right)\left(\vec{S}_{N} \cdot \vec{q}\right)$ operators. While the latter is $\mathcal{O}\left(q^{2}\right)$ suppressed, it is simultaneously enhanced by $1 /\left(m_{\pi}^{2}+\vec{q}^{2}\right)$ so that in general the two contributions are of similar size (for scattering on heavy nuclei). In this case, again, one cannot perform the direct detection analysis with just $\mathcal{O}_{4}^{N}$ or just $\mathcal{O}_{6}^{N}$. The same is true for the operators $\mathcal{Q}_{2, q}^{(6)}, \mathcal{Q}_{3, q}^{(6)}$, and $\mathcal{Q}_{10, q}^{(7)}$ that each match at leading order in chiral counting to at least two nonrelativistic operators. Therefore, a correct LO description of the DM scattering rate cannot be achieved by using only one nonrelativistic operator at a time. We explore this quantitatively in section 4 , also distinguishing the cases of light and heavy nuclei.

\section{$2.2 \quad$ Subleading corrections}

We discuss next the NLO corrections to the nonrelativistic reduction of the operators (1.3)-(1.9). The explicit expressions are given in appendix C. For each of the operators we stop at the order at which one expects the contributions from the two-nucleon currents. For most of the operators, this is $\mathcal{O}\left(q^{\nu_{\mathrm{LO}}+3}\right)$; the exceptions are the operators $\mathcal{O}_{2, q}^{(6)}, \mathcal{O}_{5, q}^{(7)}$, $\mathcal{O}_{6, q}^{(7)}$, for which the two-nucleon corrections arise at $\mathcal{O}\left(q^{\nu_{\mathrm{LO}}+1}\right)$, and the operator $\mathcal{O}_{1, q}^{(6)}$, for which the corrections are of $\mathcal{O}\left(q^{\nu_{\mathrm{LO}}+2}\right)$. Note that for $\mathcal{O}_{5, q}^{(7)}, \mathcal{O}_{6, q}^{(7)}$, and $\mathcal{O}_{1, q}^{(6)}$ the two-nucleon currents enter at the same order as the subleading corrections. Partial results for the NLO nonrelativistic reduction were derived in ref. [18], where in addition the two-nucleon corrections were considered.

Starting at subleading order there are terms that break Galilean invariance. This is a consequence of the fact that the underlying theory is Lorentz and not Galilean invariant [33]. These corrections involve the average velocity of the nucleon before and after the scattering event, $\vec{v}_{a}=\left(\vec{k}_{1}+\vec{k}_{2}\right) /\left(2 m_{N}\right)$, and lead to ten new nonrelativistic operators listed in eqs. (C.2)-(C.7).

The operators that appear at subleading order in the nonrelativistic reduction can have a qualitatively different structure from the ones that arise at LO. For instance, the vector-vector current operator $\mathcal{Q}_{1, q}^{(6)}=\left(\bar{\chi} \gamma_{\mu} \chi\right)\left(\bar{q} \gamma^{\mu} q\right)$ reduces at NLO to

$$
\begin{aligned}
\mathcal{Q}_{1, q}^{(6)} \rightarrow & F_{1}^{q / N} \mathcal{O}_{1}^{N}(1+\cdots)-\left\{\left(F_{1}^{q / N}+F_{2}^{q / N}\right) \frac{\vec{q}^{2}}{m_{\chi} m_{N}} \mathcal{O}_{4}^{N}-\left(F_{1}^{q / N}+F_{2}^{q / N}\right) \mathcal{O}_{3}^{N}\right. \\
& \left.-\frac{m_{N}}{2 m_{\chi}} F_{1}^{q / N} \mathcal{O}_{5}^{N}-\frac{m_{N}}{m_{\chi}}\left(F_{1}^{q / N}+F_{2}^{q / N}\right) \mathcal{O}_{6}^{N}+\cdots\right\} .
\end{aligned}
$$


At LO one thus has the number operator $\mathcal{O}_{1}^{N}=\mathbb{1}_{\chi} \mathbb{1}_{N}$ and no spin dependence, while the expansion to the subleading order gives in addition velocity-suppressed couplings to spin through the operators $\mathcal{O}_{4}^{N}=\vec{S}_{\chi} \cdot \vec{S}_{N}, \mathcal{O}_{3,5}^{N} \sim \vec{S}_{N, \chi} \cdot\left(\vec{v}_{\perp} \times \vec{q}\right)$, and $\mathcal{O}_{6}^{N} \sim\left(\vec{q} \cdot \vec{S}_{N}\right)\left(\vec{q} \cdot \vec{S}_{\chi}\right)$. Such corrections could have potentially important implications, if the LO expression leads to incoherent, i.e., spin-dependent scattering, while at NLO there is a contribution from the number operator $\mathcal{O}_{1}^{N}$. The latter leads to an $A^{2}$-enhanced coherent scattering rate, where $A$ is the mass number of the nucleus. For scattering on heavy nuclei with $A \sim \mathcal{O}(100)$ the chirally subleading term can potentially be the dominant contribution on nuclear scales.

There is only one operator, where this occurs, though. The tensor-tensor operator, $\mathcal{Q}_{9, q}^{(7)}=m_{q}\left(\bar{\chi} \sigma^{\mu \nu} \chi\right)\left(\bar{q} \sigma_{\mu \nu} q\right)$, leads at LO in the chiral expansion to the spin-spin interaction, $\mathcal{O}_{4}^{N}=\vec{S}_{\chi} \cdot \vec{S}_{N}$. At NLO, on the other hand, one also obtains a contribution of the form $\sim \vec{q}^{2} \mathbb{1}_{\chi} \mathbb{1}_{N}$

$$
\mathcal{Q}_{9, q}^{(7)} \rightarrow 8 F_{T, 0}^{q / N} \mathcal{O}_{4}^{N}-\left\{\frac{\vec{q}^{2}}{2 m_{N} m_{\chi}}\left(F_{T, 0}^{q / N}-F_{T, 1}^{q / N}\right) \mathcal{O}_{1}^{N}+\cdots\right\}
$$

where we do not display the other $q^{2}$-suppressed terms. For heavy nuclei the coherently enhanced contribution from $\mathcal{O}_{1}^{N}$ scales as $A \vec{q}^{2} /\left(m_{N} m_{\chi}\right) \sim \mathcal{O}(1)$ and thus the formally subleading contribution could, in principle, become important in nuclear scattering. Inspection of this particular case, however, shows that there is a relative numerical factor of 16 enhancing the leading contribution. Furthermore the coherent $\mathcal{O}\left(q^{2}\right)$ term is suppressed by $1 / m_{N} m_{\chi}$ and not simply by $1 / m_{N}^{2}$, further reducing its importance for heavy DM masses. As a result the $\mathcal{O}\left(q^{2}\right)$ terms are numerically unimportant also for the tensortensor operator. In contrast, such coherent scattering is important in $\mu \rightarrow e$ conversion, where the $m_{\chi}$ supression gets replaced by $m_{\mu}[34]$.

A potential concern is that something similar, but with a less favorable result for the numerical factors, could happen for some other operator due to the uncalculated contributions from the nonrelativistic expansion to even higher orders. However, one can easily convince oneself that this is not the case by using the parity properties of quark and DM bilinears. All the relativistic operators in eq. (1.1) that are composed from parity-odd bilinears necessarily involve the parity-odd spin operators for single-nucleon currents at each order in the chiral expansion, because one cannot form a parity-odd quantity from just two momenta - the incoming and the outgoing momentum (cf. (B.12)-(B.17)). Such operators thus never lead to coherent scattering (the argument above may need to be revisited for two-nucleon currents). This leaves us with the operators composed from parity-even bilinears only. Scalar-scalar operators and vector-vector operators lead to coherent scattering already at LO, giving tensor-tensor operator as the only left over possibility. The reduction of the tensor bilinear, eq. (B.16), gives at LO $\sim \epsilon^{\mu \nu \alpha \beta} v_{\alpha} S_{\beta}$, while at NLO one also gets, among others, the combination $v^{[\mu} q^{\nu]}$. The latter does not involve spin and leads to coherent scattering. However, due to numerical prefactors, the latter contribution is still subleading, as was shown above. 


\section{Scalar dark matter}

The above results are easily extended to the case of scalar DM. ${ }^{3}$ For relativistic scalar DM, denoted by $\varphi$, the effective interactions with the SM start at dimension six,

$$
\mathcal{L}_{\varphi}=\hat{\mathcal{C}}_{a}^{(6)} \mathcal{Q}_{a}^{(6)}+\cdots, \quad \text { where } \quad \hat{\mathcal{C}}_{a}^{(6)}=\frac{\mathcal{C}_{a}^{(6)}}{\Lambda^{2}}
$$

where ellipses denote higher dimension operators. The dimension-six operators that couple DM to quarks and gluons are

$$
\begin{array}{llrl}
\mathcal{Q}_{1, q}^{(6)} & =\left(\varphi^{*} i \stackrel{\leftrightarrow}{\partial_{\mu}} \varphi\right)\left(\bar{q} \gamma^{\mu} q\right), & & \mathcal{Q}_{2, q}^{(6)}=\left(\varphi^{*} i \stackrel{\leftrightarrow}{\partial_{\mu}} \varphi\right)\left(\bar{q} \gamma^{\mu} \gamma_{5} q\right), \\
\mathcal{Q}_{3, q}^{(6)}=m_{q}\left(\varphi^{*} \varphi\right)(\bar{q} q), & \mathcal{Q}_{4, q}^{(6)}=m_{q}\left(\varphi^{*} \varphi\right)\left(\bar{q} i \gamma_{5} q\right), \\
\mathcal{Q}_{5}^{(6)}=\frac{\alpha_{s}}{12 \pi}\left(\varphi^{*} \varphi\right) G^{a \mu \nu} G_{\mu \nu}^{a}, & \mathcal{Q}_{6}^{(6)}=\frac{\alpha_{s}}{8 \pi}\left(\varphi^{*} \varphi\right) G^{a \mu \nu} \widetilde{G}_{\mu \nu}^{a} .
\end{array}
$$

while the coupling to photons are

$$
\mathcal{Q}_{8}^{(6)}=\frac{\alpha}{12 \pi}\left(\varphi^{*} \varphi\right) F^{\mu \nu} F_{\mu \nu}, \quad \mathcal{Q}_{9}^{(6)}=\frac{\alpha}{8 \pi}\left(\varphi^{*} \varphi\right) F^{\mu \nu} \tilde{F}_{\mu \nu} .
$$

Here $\stackrel{\leftrightarrow}{\partial_{\mu}}$ is defined through $\phi_{1} \overleftrightarrow{\partial_{\mu}} \phi_{2}=\phi_{1} \partial_{\mu} \phi_{2}-\left(\partial_{\mu} \phi_{1}\right) \phi_{2}$, and $q=u, d, s$ again denote the light quarks. The strong coupling constant $\alpha_{s}$ is taken at $\mu \sim 1 \mathrm{GeV}$, and $\alpha=e^{2} / 4 \pi$ the electromagnetic fine structure constant. The operators $\mathcal{Q}_{6}^{(6)}$ and $\mathcal{Q}_{9}^{(6)}$ are CP-odd, while all the other operators are CP-even. There are also the leptonic equivalents of the operators $\mathcal{Q}_{1, q}^{(6)}, \ldots, \mathcal{Q}_{4, q}^{(6)}$, with $q \rightarrow \ell$.

At LO in chiral counting the operators coupling DM to quark and gluon currents hadronize as

$$
\begin{aligned}
& \mathcal{Q}_{1 q}^{(6)} \rightarrow 2 F_{1}^{q / N} m_{\varphi} \mathcal{O}_{1}^{N}+\mathcal{O}\left(q^{2}\right), \\
& \mathcal{Q}_{2 q}^{(6)} \rightarrow-4 F_{A}^{q / N} m_{\varphi} \mathcal{O}_{7}^{N}+\mathcal{O}\left(q^{3}\right), \\
& \mathcal{Q}_{3 q}^{(6)} \rightarrow F_{S}^{q / N} \mathcal{O}_{1}^{N}+\mathcal{O}\left(q^{2}\right), \\
& \mathcal{Q}_{4 q}^{(6)} \rightarrow F_{P}^{q / N} \mathcal{O}_{10}^{N}+\mathcal{O}\left(q^{3}\right), \\
& \mathcal{Q}_{5}^{(6)} \rightarrow F_{G} \mathcal{O}_{1}^{N}+\mathcal{O}\left(q^{2}\right), \\
& \mathcal{Q}_{6}^{(6)} \rightarrow F_{\tilde{G}} \mathcal{O}_{10}^{N}+\mathcal{O}\left(q^{3}\right) .
\end{aligned}
$$

The expressions valid to NLO in chiral counting are given in appendix D.

There are a number of qualitative differences between the cases of fermionic and scalar DM. For instance, since scalar DM does not carry a spin there is a much smaller set of operators that are generated in the nonrelativistic limit. This greatly simplifies the analysis. Furthermore, as opposed to the case of fermionic DM, there are no cases where at LO in chiral counting one would obtain incoherent scattering on nuclear spin, while at NLO in chiral counting one would have coherent scattering.

\footnotetext{
${ }^{3}$ For operators and Wilson coefficients we adopt the same notation for scalar DM as for fermionic DM. No confusion should arise as this abuse of notation is restricted to this section and appendix D.
} 


\section{Examples}

In this section we discuss several numerical examples of DM direct detection scattering. Most of the examples are for LO matching from the EFT describing DM interacting with quarks and gluons onto a theory that describes DM interacting with neutrons and protons in. At the end of this section, we will also comment on the NLO corrections. The rate $\mathcal{R}$, i.e., the expected number of events per detector mass per unit of time, is given by

$$
\frac{d \mathcal{R}}{d E_{R}}=\frac{\rho_{\chi}}{m_{A} m_{\chi}} \int_{v_{\min }} \frac{d \sigma}{d E_{R}} v f_{\oplus}(\vec{v}) d^{3} \vec{v}
$$

where $E_{R}$ is the recoil energy of the nucleus, $m_{A}$ is the mass of the nucleus, and $\rho_{\chi}$ is the local DM density. The integral is over the DM velocity $v$ in the Earth's frame with a lower bound given by $v_{\min }=\sqrt{m_{A} E_{R} / 2} / \mu_{\chi A}$, where $\mu_{\chi A}=m_{A} m_{\chi} /\left(m_{A}+m_{\chi}\right)$ is the reduced mass of DM-nucleus system. For the DM velocity distribution in the Earth's frame, $f_{\oplus}(\vec{v})$, we use the standard halo model, i.e., a distribution that in the galactic frame takes the form of an isotropic Maxwell-Boltzmann distribution with $v_{0}=254 \mathrm{~km} / \mathrm{s}$ (where $v_{0} / \sqrt{2}$ is the width of the Gaussian), truncated at the escape velocity $v_{\mathrm{esc}}=550 \mathrm{~km} / \mathrm{s}$ [35].

The DM-nucleus scattering cross section $d \sigma / d E_{R}$ in eq. (4.1) is given by

$$
\frac{d \sigma}{d E_{R}}=\frac{m_{A}}{2 \pi v^{2}} \frac{1}{\left(2 J_{\chi}+1\right)} \frac{1}{\left(2 J_{A}+1\right)} \sum_{\text {spins }}|\mathcal{M}|_{\mathrm{NR}}^{2}
$$

The nonrelativistic matrix element squared is [5]

$$
\begin{aligned}
\frac{1}{2 J_{\chi}+1} \frac{1}{2 J_{A}+1} \sum_{\text {spins }}|\mathcal{M}|_{\mathrm{NR}}^{2}= & \frac{4 \pi}{2 J_{A}+1} \sum_{\tau=0,1} \sum_{\tau^{\prime}=0,1}\left\{R_{M}^{\tau \tau^{\prime}} W_{M}^{\tau T^{\prime}}(q)+R_{\Sigma^{\prime \prime}}^{\tau \tau^{\prime}} W_{\Sigma^{\prime \prime}}^{\tau \tau^{\prime}}(q)\right. \\
& \left.+R_{\Sigma^{\prime}}^{\tau \tau^{\prime}} W_{\Sigma^{\prime}}^{\tau \tau^{\prime}}(q)+\frac{\vec{q}^{2}}{m_{N}^{2}}\left[R_{\Delta}^{\tau \tau^{\prime}} W_{\Delta}^{\tau \tau^{\prime}}(q)+R_{\Delta \Sigma^{\prime}}^{\tau \tau^{\prime}} W_{\Delta \Sigma^{\prime}}^{\tau \tau^{\prime}}(q)\right]\right\},
\end{aligned}
$$

where $J_{\chi}=1 / 2$ is the spin of DM in our examples and $J_{A}$ is the spin of the target nucleus. The nuclear response function $W_{i}$ depend on momentum exchange, $q \equiv|\vec{q}|$. The spinindependent scattering is encoded in the response function $W_{M}$ which, for instance, arises from the matrix element squared of the nuclear vector current. In the long-wavelength limit, $q \rightarrow 0, W_{M}(0)$ simply counts the number of nucleons in the nucleus giving coherently enhanced scattering, $W_{M}(0) \propto A^{2}$. The response functions $W_{\Sigma^{\prime \prime}}$ and $W_{\Sigma^{\prime}}$ have the same long-wavelength limit and measure the nucleon spin content of the nucleus. $W_{\Delta}$ measures the nucleon angular momentum content of the nucleus, while $W_{\Delta \Sigma^{\prime}}$ is the interference term. These functions roughly scale as $W_{M} \sim \mathcal{O}\left(A^{2}\right)$, and $W_{\Sigma^{\prime}}, W_{\Sigma^{\prime \prime}}, W_{\Delta}, W_{\Delta \Sigma^{\prime}} \sim \mathcal{O}(1)$, where the actual size depends on the particular nucleus and can differ significantly from one nucleus to another. The prefactors $R_{i}$ encode the dependence on the $c_{i}^{N}\left(q^{2}\right)$ coefficients, eq. (1.10), and on kinematical factors. For instance, the coefficient of the coherently enhanced term is

$$
R_{M}^{\tau \tau^{\prime}}=c_{1}^{\tau} c_{1}^{\tau^{\prime}}+\frac{1}{4}\left[\frac{\vec{q}^{2}}{m_{N}^{2}} c_{11}^{\tau} c_{11}^{\tau^{\prime}}+\vec{v}_{T}^{\perp 2}\left(c_{8}^{\tau} c_{8}^{\tau^{\prime}}+\frac{\vec{q}^{2}}{m_{N}^{2}} c_{5}^{\tau} c_{5}^{\tau^{\prime}}\right)\right]
$$




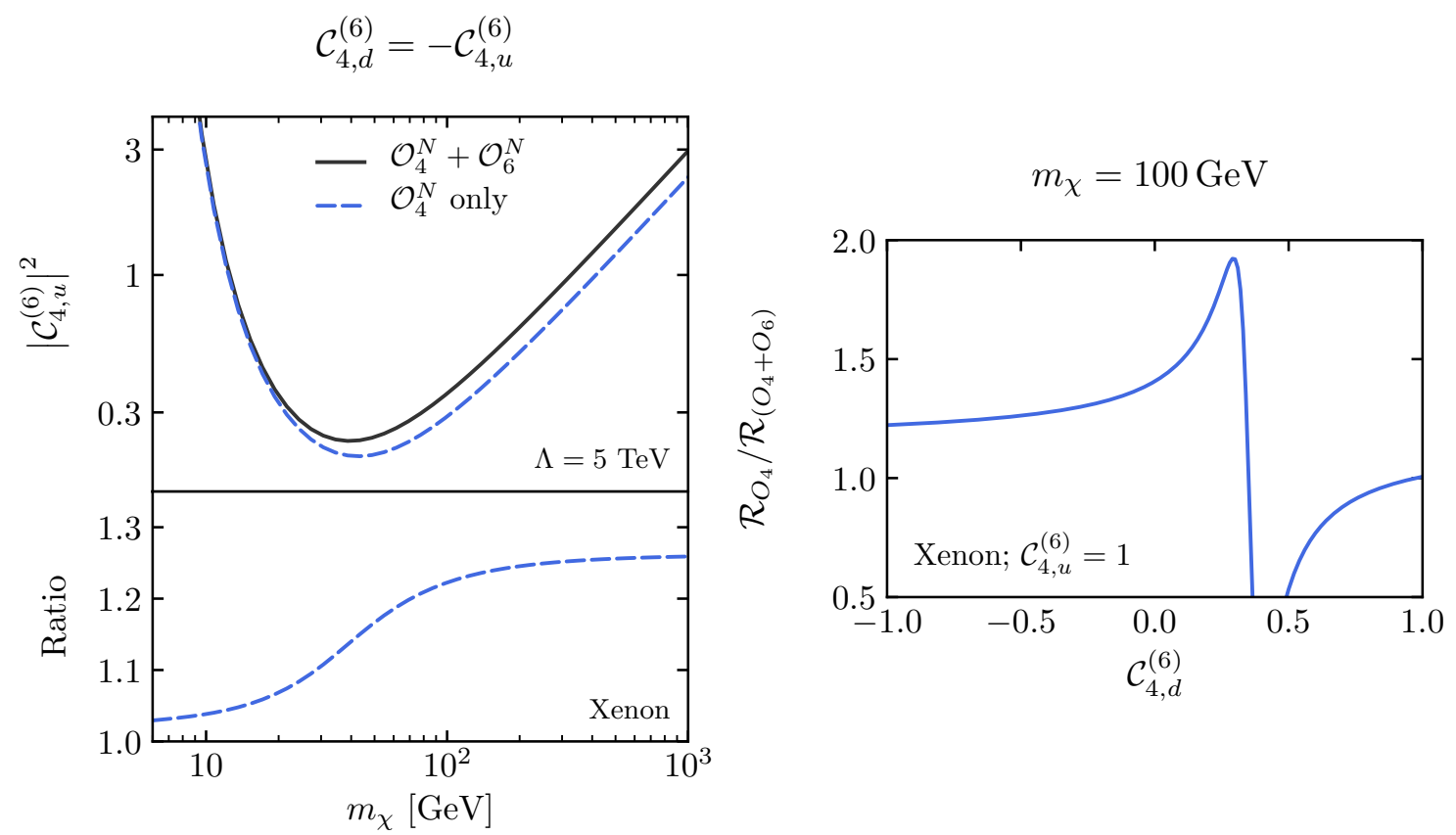

Figure 4. Left panel: an illustration of Xe target bounds on the Wilson coefficients $\mathcal{C}_{4, u}^{(6)}=-\mathcal{C}_{4, d}^{(6)}$ for the interaction operator $\left(\bar{\chi} \gamma^{\mu} \gamma_{5} \chi\right)\left(\bar{q} \gamma_{\mu} \gamma_{5} q\right)$ assuming opposite couplings to the $u$ and $d$ quarks. The correct, chirally leading, treatment of the induced spin-dependent scattering with both $\mathcal{O}_{4}^{N}=$ $\vec{S}_{\chi} \cdot \vec{S}_{N}$ and $\mathcal{O}_{6}^{N} \propto\left(\vec{S}_{\chi} \cdot \vec{q}\right)\left(\vec{S}_{N} \cdot \vec{q}\right)$ operators (black solid line) is compared to that of $\mathcal{O}_{4}^{N}$ only (blue dashed line). The ratio of the two is shown in the bottom plot. Right panel: the ratio of the $\mathcal{O}_{4}$ contribution to the rate over the total rate as a function of the Wilson coefficient $\mathcal{C}_{4, d}^{(6)}$ for a fixed value of $\mathcal{C}_{4, u}^{(6)}=1$, taking $m_{\chi}=100 \mathrm{GeV}$.

where $\vec{v}_{T}^{\perp}=\vec{v}-\vec{q} /\left(2 \mu_{\chi A}\right) \sim 10^{-3}$. The sum in eq. (4.3) is over isospin values $\tau=0,1$ which are related to the proton and neutron coefficients by $c_{i}^{0}=\left(c_{i}^{p}+c_{i}^{n}\right) / 2, c_{i}^{1}=\left(c_{i}^{p}-c_{i}^{n}\right) / 2$. The remaining $R_{i}^{\tau \tau^{\prime}}$ can be found in [5]. Using these expressions for $R_{M}^{\tau \tau^{\prime}}$ together with our expressions for the hadronization of the EFT operators, eqs. (2.19)-(2.34), which give the coefficients $c_{i}^{\tau}$ (see appendix E), we are now in a position to obtain the rates in a DM direct detection experiment assuming a particular interaction of DM with the visible sector.

In the following, when we calculate the scattering rate and plot the bound on the squared UV Wilson coefficients, we restrict the integral over the recoil energy. To approximate the LUX sensitivity region we integrate over $E_{R} \in[3,50] \mathrm{keV}$ for Xenon [29]. To approximate PICO's [28] sensitivity we integrated over $E_{R}>3.3 \mathrm{keV}$ for Fluorine - see figures 1 and 5. To obtain total rates for scattering on Xenon, we assume an exposure of $5000 \mathrm{~kg} \cdot \mathrm{yr}$ which is representative of the next generation two-phase liquid Xenon detectors. Since Xenon has eight naturally occurring stable isotopes, we sum over them weighted by their natural abundances.

The first few examples, shown in figures 4,5 , and 6 , illustrate that one cannot always take the long wavelength limit, $q \rightarrow 0$, in the calculation of DM scattering rates when matching from $\mathcal{L}_{\chi}$ to $\mathcal{L}_{\mathrm{NR}}$. This problem is well known for the description of DM scat- 

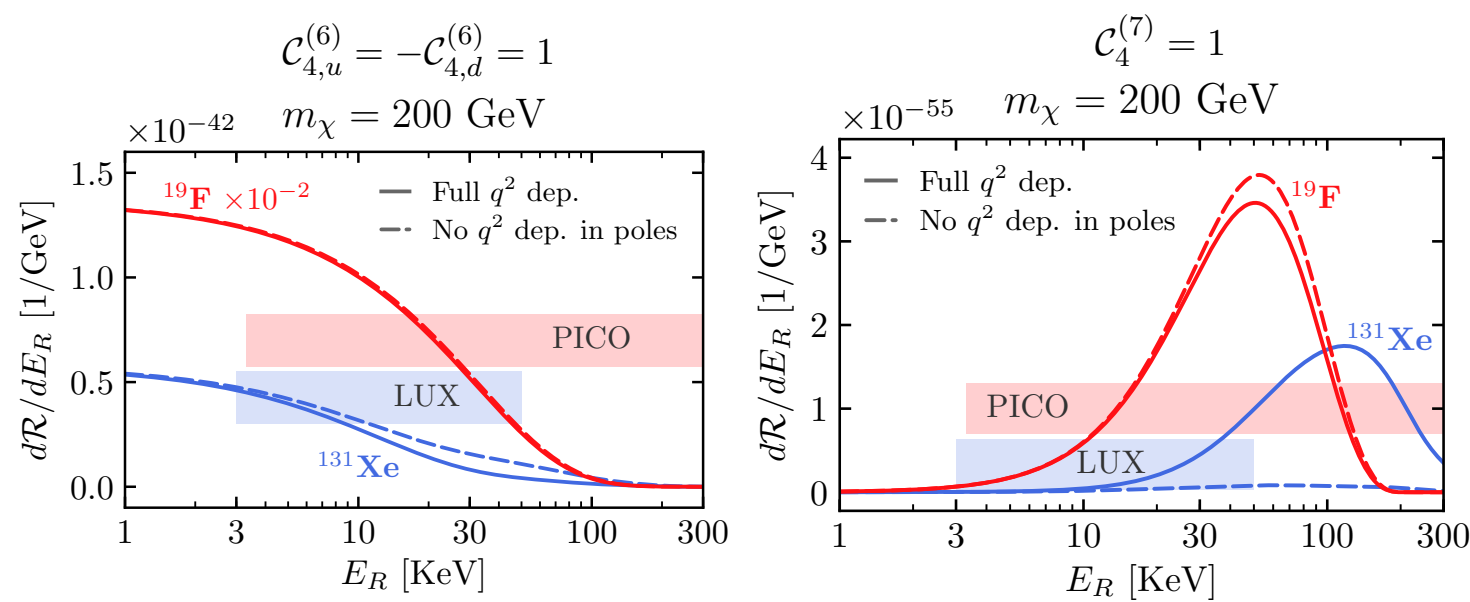

Figure 5. The differential event rate, $d \mathcal{R} / d E_{R}$, as a function of the recoil energy, $E_{R}$, for scattering on Xenon (blue) and Fluorine (red) for $\mathcal{Q}_{4, q}^{(6)}$ and $\mathcal{Q}_{4}^{(7)}$ in the left and right panels respectively. In both panels, the solid curves include the full $q^{2}$ dependence in the form factor $F_{\tilde{G}}\left(q^{2}\right)$ while the dashed lines include only the zero recoil limit, $F_{\tilde{G}}(0)$. The shaded regions depict the approximate ranges of experimental sensitivity for the LUX (blue) and PICO (red) experiments.

tering on whole nuclei, the effect described by the momentum dependence of the nuclear response functions. For instance, a momentum exchange of $q=100 \mathrm{MeV}$ already leads to decoherence and thereby reduces the spin-independent nuclear form factor $W_{M}$ by $\sim 20 \%$ $(\sim 60 \%)$ for scattering on Fluorine (Xenon). Our examples show a different effect, namely that sometimes the momentum dependence cannot be neglected even when considering the scattering on a single neutron and/or proton. This effect is described by the momentum dependence of the coefficients $c_{i}^{\tau \tau^{\prime}}$. Since nucleons have smaller spatial dimensions than nuclei, the effects of the momentum dependence of $c_{i}^{\tau \tau^{\prime}}$ are expected to be smaller than those of the momentum dependence of $W_{i}^{\tau \tau^{\prime}}$. However, because the pseudoscalar hadronic currents contain pion poles, the corrections due to non-zero momentum in the corresponding $c_{i}^{\tau \tau^{\prime}}$ are of $\mathcal{O}\left(\vec{q}^{2} / m_{\pi}^{2}\right)$ and can be large.

The effect of such contributions for scattering on Xenon is shown in figure 4 . The chirally leading hadronization of the axial-axial operator $\left(\bar{\chi} \gamma^{\mu} \gamma_{5} \chi\right)\left(\bar{q} \gamma_{\mu} \gamma_{5} q\right)$ contains two nonrelativistic operators, $\mathcal{O}_{4}^{N}=\vec{S}_{\chi} \cdot \vec{S}_{N}$ and $\mathcal{O}_{6}^{N} \propto\left(\vec{S}_{\chi} \cdot \vec{q}\right)\left(\vec{S}_{N} \cdot \vec{q}\right)$. The latter is momentum suppressed but comes with a pion-pole enhanced coefficient, see eq. (2.38), and thus gives an $\mathcal{O}(1)$ contribution to the scattering rate through interference with $\mathcal{O}_{4}^{N}$. The left panel in figure 4 shows a bound (solid black line) on the relativistic Wilson coefficient $\mathcal{C}_{4, q}^{(6)}$ assuming equal and opposite couplings to the $u$ and $d$ quarks, and a vanishing coupling to $s$ quarks. ${ }^{4}$ This is compared with the extraction of the bound on $\mathcal{C}_{4, q}^{(6)}$ where the contribution of $\mathcal{O}_{6}^{N}$ is neglected (dashed blue line). The two bounds coincide for small $m_{\chi}$ since in that case the exchanged momenta are small which parametrically suppresses the $\mathcal{O}_{6}^{N}$ contribution. The relative difference then grows with $m_{\chi}$ up to $m_{\chi} \sim m_{A}$ (see lower plot in figure 4 left), and is typically of $\mathcal{O}(20 \%-50 \%)$, figure 4 (right), confirming the expectation from

\footnotetext{
${ }^{4}$ In fact, we show a bound on $\left|\mathcal{C}_{4, q}^{(6)}\right|^{2}$ since this is directly proportional to the scattering rate.
} 

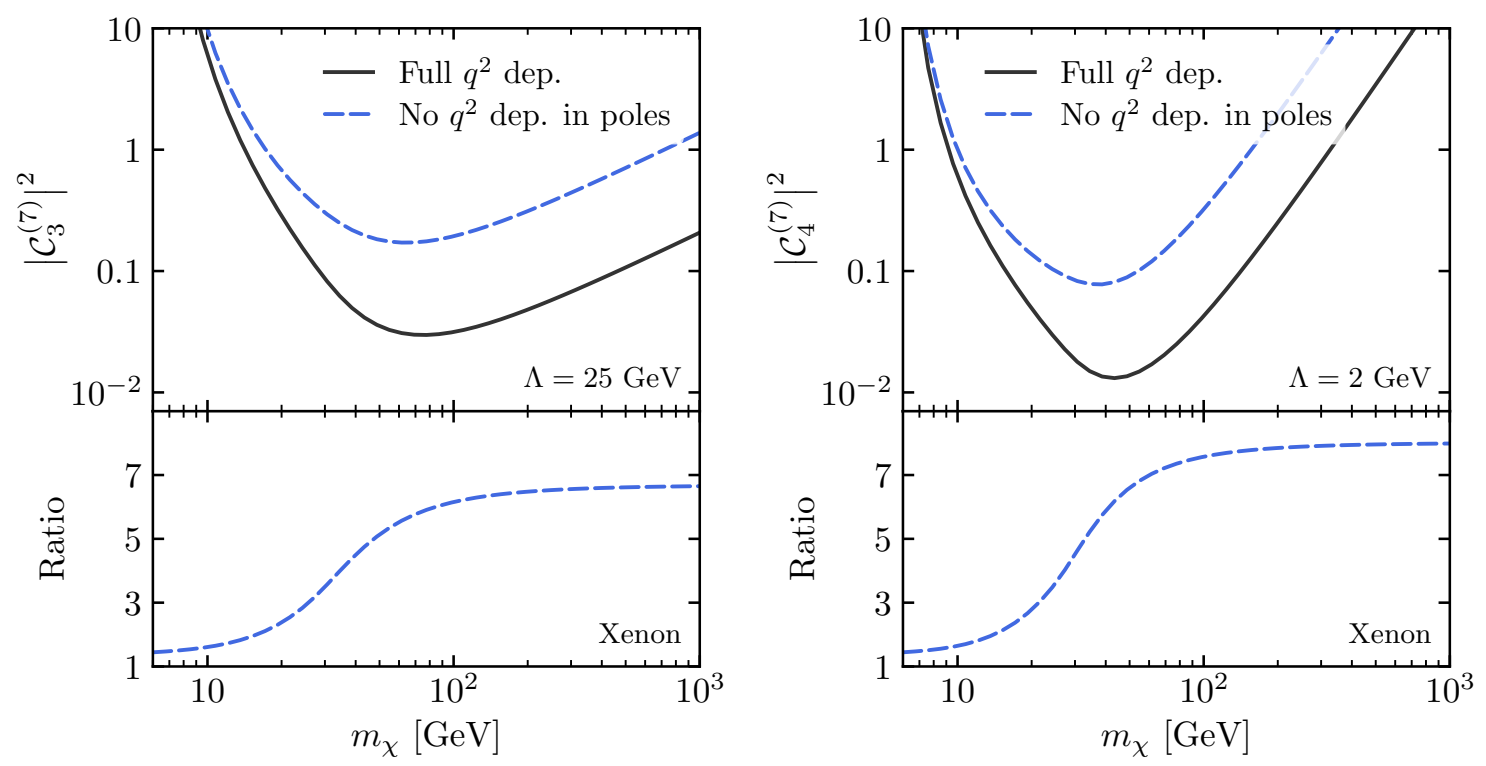

Figure 6. Comparison between the bounds on the squared Wilson coefficients of the UV operators $\mathcal{Q}_{3}^{(7)}$ (left panel) and $\mathcal{Q}_{4}^{(7)}$ (right panel) for scattering on a Xenon target. The dashed and solid curves correspond to the bound with and without meson exchanges respectively. The lower plots show the ratio of the bounds without and with the inclusion of meson exchange.

chiral counting that the correction is $\mathcal{O}(1)$ unless there are cancellations in one of the two contributions. For instance, the $\mathcal{O}_{4}^{N}$ contribution is suppressed for $\mathcal{C}_{4, d}^{(6)} \simeq \mathcal{C}_{4, u}^{(6)} / 2$ and a DM mass $m_{\chi}=100 \mathrm{GeV}$. Independent of the DM mass, however, the pion pole is completely absent for $\mathcal{C}_{4, d}^{(6)}=\mathcal{C}_{4, u}^{(6)}$, and the $\mathcal{O}_{6}^{N}$ contribution to the scattering rate becomes negligible.

Furthermore, the contribution from $\mathcal{O}_{6}^{N}$ is expected to be negligible for scattering on light nuclei since the exchanged momenta are small, see figure 1 . We have explicitly checked this for scattering on Fluorine, with the corresponding effect on $d \mathcal{R} / d E_{R}$ shown in figure 5 (left) for $m_{\chi}=200 \mathrm{GeV}$. For scattering on ${ }^{19} F$ the predictions with (solid red line) and without $\mathcal{O}_{6}^{N}$ (dashed red line) essentially coincide while for scattering on Xenon there is a large distortion of the spectrum in the signal region for LUX.

The effect of pion exchange is even more pronounced if DM couples to the visible sector through parity-odd gluonic operators, i.e., if the operators in eq. (1.6) dominate. In figure 6 , we show the bounds on the Wilson coefficients of the $\mathcal{Q}_{3}^{(7)} \propto \bar{\chi} \chi G \tilde{G}$ operator (left panel), and of the operator $\mathcal{Q}_{4}^{(7)} \propto \bar{\chi} i \gamma_{5} \chi G \tilde{G}$ (right panel). The corresponding nucleon form factor has a schematic form

$$
F_{\tilde{G}}(q) \sim \sum_{i} \frac{\Delta q_{i}}{m_{q_{i}}}+\delta m \frac{q^{2}}{m_{\pi, \eta}^{2}-q^{2}},
$$

where $\Delta q_{i}$ is the axial charge of quark $q_{i}$ and the $\delta m$ coefficient is the size of isospin breaking for pion exchange and the SU(3)-flavor breaking for eta meson exchange, see eq. (A.42). Note that isospin breaking is $\mathcal{O}(1)$ for the matrix element of the QCD anomaly term $\alpha_{s} /(8 \pi) G \tilde{G}$ while it is of $\mathcal{O}(10 \%)$ for all other matrix elements [36]. The importance 

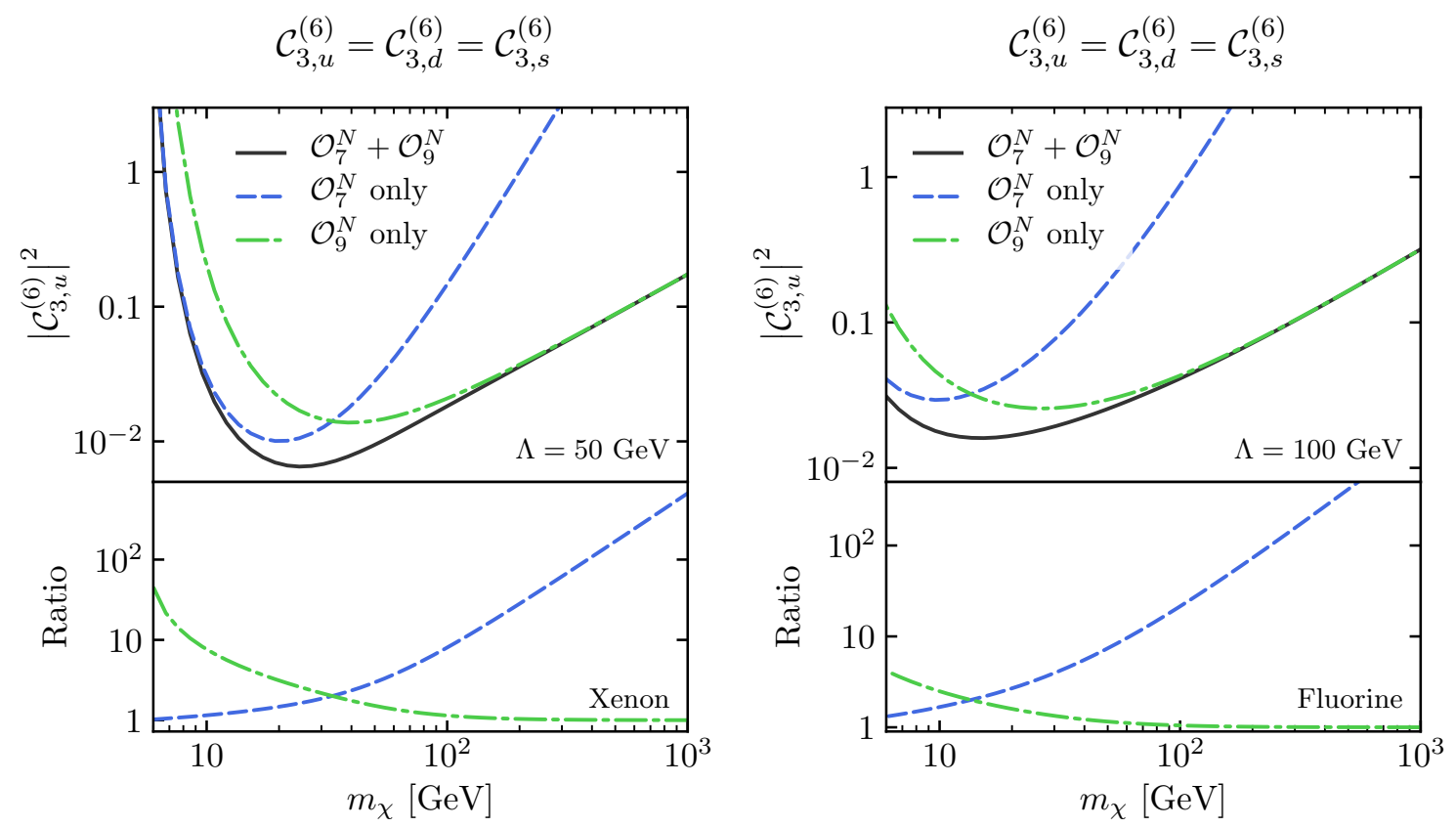

Figure 7. The bounds on the squared Wilson coefficient of the $\mathcal{Q}_{3, q}^{(6)}=\left(\bar{\chi} \gamma^{\mu} \chi\right)\left(\bar{q} \gamma_{\mu} \gamma_{5} q\right)$ operator from scattering on Xenon (left) and Fluorine (right), taking into account only $\mathcal{O}_{7}^{N}$ (dashed blue line), only $\mathcal{O}_{9}^{N}$ operator (dot-dashed green line), and both (solid black). The coupling to all three light quarks are set equal to each other.

of isospin-breaking but pion-pole enhanced contributions is reflected in the DM scattering rates. The bounds on the Wilson coefficients $\mathcal{C}_{3,4}^{(7)}$ in figure 6 , obtained with the correct full form factor dependence, are depicted with solid black lines. For weak-scale DM masses they can be even up to an order of magnitude stronger than the bounds obtained by only using the zero recoil form factor, $F_{\tilde{G}}(0)$ (dashed blue lines). Ignoring the leading $q^{2}$-dependence in $F_{\tilde{G}}$ also leads to a large distortion of the shape in $d \mathcal{R} / d E_{R}$ as shown in figure 5 (right) for the $\mathcal{Q}_{4}^{(7)}$ operator and $m_{\chi}=200 \mathrm{GeV}$. In this case, there is a visible change in the shape of the differential rate even for scattering on Fluorine, despite small momenta exchanges. The effect is striking for the scattering on Xenon where momenta exchanges are typically larger. For the $\mathcal{Q}_{3}^{(7)}$ operator, the distortion is slightly smaller, but otherwise comparable to the one shown.

For the $\mathcal{Q}_{4, q}^{(6)}$ and $\mathcal{Q}_{4}^{(7)}$ operators discussed above and shown for scattering on Xenon in figures 4 and 6 respectively, the $\vec{q}^{2}$ dependence in the meson poles is negligible for scattering on Fluorine. To understand this it is useful to consider the differential scattering rate as a function of the recoil energy. This is shown in figure 5 for a fixed DM mass of $200 \mathrm{GeV}$. For both interactions, the $E_{R}$ spectra for Fluorine do not differ significantly when the $\vec{q}^{2}$ dependence in the meson poles is neglected since a given value of $E_{R}$ results in a momentum transfer $\vec{q}^{2} / m_{A}$ that is smaller by an order of magnitude in Fluorine than in Xenon.

A qualitatively different example is given in figure 7 which shows the bounds on the Wilson coefficient $\mathcal{C}_{3, q}^{(6)}$ as function of $m_{\chi}$ for scattering on Xenon and Fluorine. The vector- 

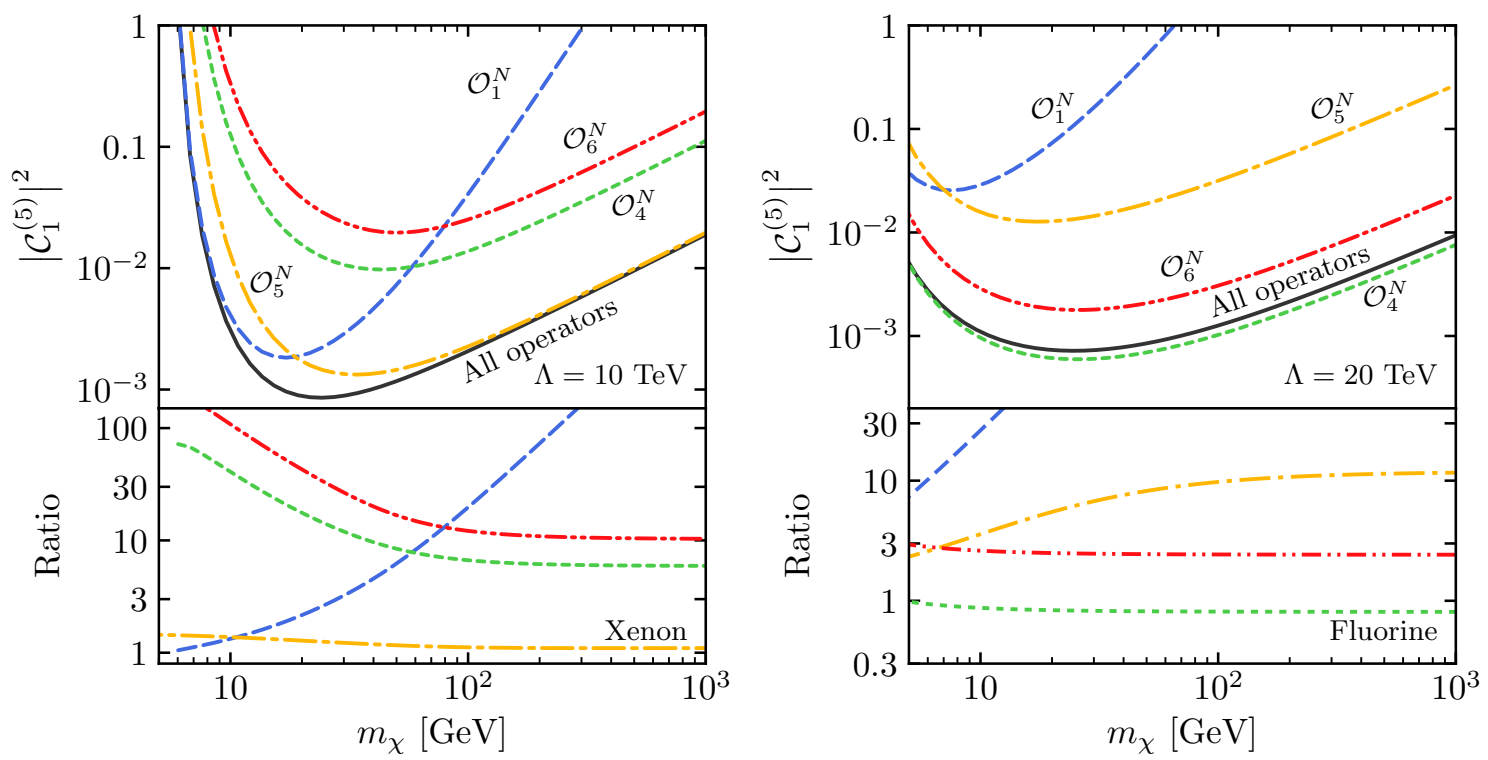

Figure 8. The bound on the squared Wilson coefficient of the magnetic dipole operator $\mathcal{Q}_{1}^{(5)}$. The left (right) panel shows the scattering on Xenon (Fluorine). The EFT scale was fixed to 10 and $20 \mathrm{TeV}$ for scattering on Xenon and Fluorine respectively. For both targets, the solid curve corresponds to the total rate while the dashed, dotted, dash-dotted and dash-double-dotted curves correspond to turning one non-relativistic operator at a time.

axial operator, $\mathcal{Q}_{3, q}^{(6)}=\left(\bar{\chi} \gamma^{\mu} \chi\right)\left(\bar{q} \gamma_{\mu} \gamma_{5} q\right)$, eq. (1.4), matches onto two non-relativistic operators, $\mathcal{O}_{7}^{N} \propto \vec{S}_{N} \cdot \vec{v}_{\perp}$ and $\mathcal{O}_{9}^{N} \propto \vec{S}_{\chi} \cdot\left(\vec{q} \times \vec{S}_{N}\right)$. At leading order in chiral power counting, the hadronization of the axial quark-current in $\mathcal{Q}_{3, q}^{(6)}$ is described by one form factor at zero recoil, $F_{A}^{q / N}(0)$, see eq. (2.23). This form factor is therefore a common coefficient in the matching onto both $\mathcal{O}_{7}^{N}$ and $\mathcal{O}_{9}^{N}$. Nevertheless, the contribution due to $\mathcal{O}_{9}^{N}$ is suppressed by an additional power of the DM mass (i.e, two powers in the rate) and thus becomes subleading for larger DM masses. Since the contributions are correlated yet scale differently with $m_{\chi}$, it is crucial to consider both non-relativistic operators when setting bounds from direct detection experiments (see, e.g., [37]).

The non-trivial interplay between different non-relativistic operators can also be seen in the case of dipole interaction, $\mathcal{Q}_{1}^{(5)}$, shown in figure 8. This operator matches onto four NR operators $\mathcal{O}_{1}^{N}, \mathcal{O}_{4}^{N}, \mathcal{O}_{5}^{N}, \mathcal{O}_{6}^{N}$, see eq. (2.19). Out of these, two are coherently enhanced, $\mathcal{O}_{1}^{N}=\mathbb{1}_{\chi} \mathbb{1}_{N}$ and $\mathcal{O}_{5}^{N} \propto \vec{S}_{\chi} \cdot\left(\vec{v}_{\perp} \times \vec{q}\right) \mathbb{1}_{N}$. One expects these two to dominate for heavier nuclei, as shown explicitly for Xenon in figure 8 (left). The $\mathcal{O}_{5}^{N}$ operator is enhanced by an explicit photon pole prefactor, $1 / \vec{q}^{2}$, which overcomes the velocity suppression and leads to its dominance over all other contributions. The contribution from the $\mathcal{O}_{1}^{N}$ operator, on the other hand, is local and is suppressed for heavy DM by a $1 / m_{\chi}$ factor. Its contribution is, therefore, relevant only for light DM.

For DM scattering on lighter nuclei, the situation is more involved. The coherent enhancement is not as large and does not overcome the velocity suppression in $\mathcal{O}_{5}^{N}$ even 
though it is accompanied by the $1 / \vec{q}^{2}$ enhancement. For $\mathcal{O}_{1}^{N}$, the factor of $1 / m_{\chi}$ still suppresses its contribution, particularly for $m_{\chi} \gtrsim \mathcal{O}(10) \mathrm{GeV}$. For Fluorine the leading contributions thus come from incoherent scattering due to the spin-dependent $\mathcal{O}_{4}^{N}$ and $\mathcal{O}_{6}^{N}$ operators. Parametrically, they scale in the same way (the $\vec{q}^{2}$ factor in $\mathcal{O}_{6}^{N}$ is cancelled by the $1 / \vec{q}^{2}$ in its Wilson coefficient). Numerically, however, the contribution from $\mathcal{O}_{4}^{N}$ is about three times larger. Furthermore, the contributions have opposite signs and interfere destructively as can be seen in the right panel of figure 8 , with $\mathcal{O}_{4}^{N}$ giving a stronger bound than the sum of all operators.

Finally, we turn our attention to the NLO corrections. The chiral counting of the expansion in powers of $q^{2}$ is well motivated but does not capture all effects. For instance, the NLO corrections in chiral counting can become important if coherently enhanced operators appear at NLO when there were none at LO. This is indeed the case for the tensor operator $\mathcal{Q}_{9, q}^{(7)}$ where two coherently enhanced operators, $\mathcal{O}_{1}^{N}$ and $\mathcal{O}_{5}^{N}$, appear at NLO in the expansion, while at LO no coherently enhanced operators are present. However, even for Xenon, the coherent enhancement is not enough to compensate for the $\vec{q}^{2} / m_{N} m_{\chi}$ suppression accompanied by a relative factor of $1 / 16$, and thus the resulting correction is of $\mathcal{O}(5 \%)$. A similar coherently enhanced contribution appears for $\mathcal{Q}_{10, q}^{(7)}$ operator at $\mathcal{O}\left(q^{4}\right)$ and is thus completely negligible.

\section{Conclusions}

In this article we derived the expressions for the matching of an EFT for DM interacting with quarks and gluons, described by the effective Lagrangian $\mathcal{L}_{\chi}$ in eq. (1.1), to an EFT described by the Lagrangian $\mathcal{L}_{\mathrm{NR}}$ for nonrelativistic DM interacting with nonrelativistic nucleons, eq. (1.10). The latter is then used as an input to the description of DM interactions with nuclei, described in terms of nuclear response functions. The rationale underlying our work is the organization of different contributions according to chiral power counting, i.e., in terms of an expansion in $\vec{q}^{2} / \Lambda_{\text {ChEFT }}^{2}$ and counting $q \sim m_{\pi}$. Within this framework one can make the following observations: (i) for LO expressions one needs nonrelativistic operators with up to two derivatives, since they can be enhanced by pion poles giving a contribution of the order of $\vec{q}^{2} /\left(m_{\pi}^{2}+\vec{q}^{2}\right) \sim \mathcal{O}(1)$; (ii) not all of the nonrelativistic operators $\mathcal{O}_{i}^{N}$ with two derivatives are generated when starting from an EFT for DM interacting with quarks and gluons; (iii) a single relativistic operator $\mathcal{Q}_{i}^{(d)}$ can generate several nonrelativistic operators $\mathcal{O}_{i}^{N}$ with momentum-dependent coefficients already at LO; (iv) interactions of DM with two-nucleon currents are chirally suppressed (barring cancellations of LO terms), justifying our treatment of DM interacting with only single-nucleon currents.

We worked to next-to-leading order in the chiral expansion, but also discussed separately the expressions for the leading-order matching. At LO the scattering of DM on nucleons only depends on the DM spin $\vec{S}_{\chi}$, the nucleon spin $\vec{S}_{N}$, the momentum exchange $\vec{q}$, and the averaged relative velocity between DM and nucleon before and after scattering, $\vec{v}_{\perp}$. All these quantities are Galilean invariant. At NLO in chiral counting the expressions depend in addition on the averaged velocity of nucleon before and after scattering, $\vec{v}_{a}$. This dependence on Galilean non-invariant quantities such as $\vec{v}_{a}$ is expected, since the 
underlying theory is Lorentz and not Galilean invariant. Because of the dependence on $\vec{v}_{a}$ the NLO expressions require an expanded nonrelativistic operator basis, with the new operators listed in appendix C.

Numerically the NLO corrections are always small, at the level of $\mathcal{O}\left(\vec{q}^{2} / m_{N}^{2}\right)$ or a few percent, unless one fine tunes the cancellation of LO expressions. This result is nontrivial for the partonic tensor-tensor operator $Q_{9, q}^{(7)}=m_{q}\left(\bar{\chi} \sigma^{\mu \nu} \chi\right)\left(\bar{q} \sigma_{\mu \nu} q\right)$, since in that case the LO term is spin-dependent, while the NLO corrections contain a spin-independent contribution that is coherently enhanced. In principle this could compete with the LO term. However, due to fortuitous numerical factors, it remains subleading.

While our results were obtained by assuming that the mediators between the DM and the visible sector are heavy, with masses above several hundred $\mathrm{MeV}$, the formalism can be easily changed to accommodate lighter mediators. In this case the mediators cannot be integrated out, but lead to an additional momentum dependence of the coefficients in the nonrelativistic Lagrangian $\mathcal{L}_{\mathrm{NR}}$, eq. (1.10), and potentially to a modified counting of chirally leading and subleading terms. The details of the latter would depend on the specifics of the underlying DM theory.

As a side-result, our expressions show that from the particle physics point of view it is more natural to interpret the results of direct detection experiments in terms of an EFT where DM interacts with quarks and gluons, eq. (1.1). The reason is that several of the partonic operators in $\mathcal{L}_{\chi}$ match to more than one nonrelativistic operator already at leading order in chiral counting. In such cases it is then hard to justify singling out just one nonrelativistic operator in the analysis of direct detection experimental results.

The situation becomes even more complicated if the partonic operator matches onto several nuclear operators with different momentum dependence, since in the experiments one integrates over a range of momenta. A cautionary example of wider phenomenological interest is the case of the axial-axial partonic operator, $Q_{4, q}^{(6)}=\left(\bar{\chi} \gamma_{\mu} \gamma_{5} \chi\right)\left(\bar{q} \gamma^{\mu} \gamma_{5} q\right)$, which induces spin-dependent scattering. At leading chiral order this is described by a combination of the $\mathcal{O}_{4}^{N}=\vec{S}_{\chi} \cdot \vec{S}_{N}$ and $\mathcal{O}_{6}^{N} \sim\left(\vec{S}_{\chi} \cdot \vec{q}\right)\left(\vec{S}_{N} \cdot \vec{q}\right)$ nonrelativistic operators. Naively the latter is momentum suppressed. We find that this is true for DM scattering on light nuclei, such as Fluorine, where the contribution from $\mathcal{O}_{6}^{N}$ is in fact unimportant, since the momenta exchanges are in this case small, $q \ll m_{\pi}$. However, for DM scattering on heavy nuclei, such as Xenon, the $\mathcal{O}_{6}^{N}$ operator does give an $\mathcal{O}(1)$ correction due to its enhancement by a pion pole, in line with the expectations from chiral counting. Thus, in general both contributions from $\mathcal{O}_{4}^{N}$ and $\mathcal{O}_{6}^{N}$ need to be kept.

The flip side of the above discussion is the question: are there models of DM where only $\mathcal{O}_{4}^{N}$ or only $\mathcal{O}_{6}^{N}$ operator is generated? For these two operators the answer is yes. At leading chiral order the partonic operator $\mathcal{Q}_{9, q}^{(7)}=m_{q}\left(\bar{\chi} \sigma^{\mu \nu} \chi\right)\left(\bar{q} \sigma_{\mu \nu} q\right)$ only generates $\mathcal{O}_{4}^{N}$, while the partonic operators $\mathcal{Q}_{4}^{(7)} \sim\left(\bar{\chi} i \gamma_{5} \chi\right) G \widetilde{G}, \mathcal{Q}_{8, q}^{(7)}=m_{q}\left(\bar{\chi} i \gamma_{5} \chi\right)\left(\bar{q} i \gamma_{5} q\right)$ only induce the operator $\mathcal{O}_{6}^{N}$. But the same is not true in general. For a number of other nonrelativistic operators $-\mathcal{O}_{7}^{N}, \mathcal{O}_{8}^{N}, \mathcal{O}_{9}^{N}$ and $\mathcal{O}_{12}^{N}$ - there is no partonic level operator that would induce just one of these. All of them are always accompanied by other nonrelativistic operators when matching from $\mathcal{L}_{\chi}$ to $\mathcal{L}_{\mathrm{NR}}$. For these nonrelativistic operators switching on just one 
operator at the time when analysing direct detection data thus does not make much sense from the microscopic point of view. Furthermore, the nonrelativistic operators $\mathcal{O}_{2}^{N}, \mathcal{O}_{3}^{N}$, $\mathcal{O}_{13}^{N}, \mathcal{O}_{14}^{N}, \mathcal{O}_{15}^{N}, \mathcal{O}_{2 b}^{N}$ are never generated as leading operators when starting from a UV theory of DM. They enter only as subleading corrections in the scattering rates, and can always be neglected (as can the other nine nonrelativistic operators listed in appendix $\mathrm{C}$ that have already never been considered).

In conclusion, we advocate the use of partonic level EFT basis eqs. (1.2)-(1.9) as a phenomenologically consistent way of interpreting direct detection data. Including all the variations due to quark flavor assignments there are 34 operators in total, which is not much more than the 28 nonrelativistic operators used at present. Moreover, using the partonic level EFT also has the added benefit of providing a simple connection with the use of EFT in collider searches for dark matter, via straight-forward renormalization-group evolution.

\section{Acknowledgments}

We thank Christian Bauer, Eugenio Del Nobile, Ulrich Haisch, Matthew McCullough, Paolo Panci, Mikhail Solon, and Alessandro Strumia for useful discussions. FB is supported by the Science and Technology Facilities Council (STFC). JZ is supported in part by the U.S. National Science Foundation under CAREER Grant PHY-1151392 and by the DOE grant de-sc0011784. BG is supported in part by the U.S. Department of Energy under grant DE-SC0009919.

\section{A Values of the nucleon form factors}

Below we give the values for the form factors $F_{i}^{p / q}$ for proton external states, while the corresponding values for neutrons are obtained through exchange of $p \rightarrow n, u \leftrightarrow d$.

\section{A.1 Vector current}

The general matrix element of the vector current (2.2) is parameterized by two sets of form factors $F_{1}^{q / N}\left(q^{2}\right)$ and $F_{2}^{q / N}\left(q^{2}\right)$. For the LO expressions we only need their values evaluated at $q^{2}=0$, while for the subleading expression (C.9) we also need $F_{1}^{\prime q / N}(0)$.

At zero momentum exchange the vector currents count the number of valence quarks in the nucleon. Hence, the normalization of the Dirac form factors for the proton is

$$
F_{1}^{u / p}(0)=2, \quad F_{1}^{d / p}(0)=1, \quad F_{1}^{s / p}(0)=0 .
$$

The Pauli form factors $F_{2}^{q / N}(0)$ describe the contributions of quarks to the anomalous magnetic moments of the nucleons,

$$
\begin{aligned}
& a_{p}=\frac{2}{3} F_{2}^{u / p}(0)-\frac{1}{3} F_{2}^{d / p}(0)-\frac{1}{3} F_{2}^{s / p}(0) \approx 1.793, \\
& a_{n}=\frac{2}{3} F_{2}^{u / n}(0)-\frac{1}{3} F_{2}^{d / n}(0)-\frac{1}{3} F_{2}^{s / n}(0) \approx-1.913 .
\end{aligned}
$$


Using the strange magnetic moment [38] (see also [39])

$$
F_{2}^{s / p}(0)=-0.064(17)
$$

one gets, using isospin symmetry,

$$
\begin{aligned}
& F_{2}^{u / p}(0)=2 a_{p}+a_{n}+F_{2}^{s / p}(0)=1.609(17), \\
& F_{2}^{d / p}(0)=2 a_{n}+a_{p}+F_{2}^{s / p}(0)=-2.097(17) .
\end{aligned}
$$

For the slope of $F_{1}^{q / N}\left(q^{2}\right)$ at $q^{2}=0$ one obtains [8]

$$
\begin{aligned}
& F_{1}^{\prime u / p}(0)=\frac{1}{6}\left(2\left[r_{E}^{p}\right]^{2}+\left[r_{E}^{n}\right]^{2}+r_{s}^{2}\right)-\frac{1}{4 m_{N}^{2}}\left(2 a_{p}+a_{n}\right)=5.57(9) \mathrm{GeV}^{-2}, \\
& F_{1}^{\prime d / p}(0)=\frac{1}{6}\left(\left[r_{E}^{p}\right]^{2}+2\left[r_{E}^{n}\right]^{2}+r_{s}^{2}\right)-\frac{1}{4 m_{N}^{2}}\left(a_{p}+2 a_{n}\right)=2.84(5) \mathrm{GeV}^{-2}, \\
& F_{1}^{\prime s / p}(0)=\frac{1}{6} r_{s}^{2}=-0.018(9) \mathrm{GeV}^{-2}
\end{aligned}
$$

using the values $\left[r_{E}^{p}\right]^{2}=0.7658(107) \mathrm{fm}^{2}[35,40],\left[r_{E}^{n}\right]^{2}=-0.1161(22) \mathrm{fm}^{2}[35]$, and $r_{s}^{2}=-0.0043(21) \mathrm{fm}^{2}[38]$.

Above we used the definitions for the proton and neutron matrix elements of the electromagnetic current,

$$
\left\langle N^{\prime}\left|J_{\mathrm{em}}^{\mu}\right| N\right\rangle=\bar{u}_{N}^{\prime}\left[F_{1}^{N}\left(q^{2}\right) \gamma^{\mu}+\frac{i}{2 m_{N}} F_{2}^{N}\left(q^{2}\right) \sigma^{\mu \nu} q_{\nu}\right] u_{N}, \quad N=p, n,
$$

where $J_{\mathrm{em}}^{\mu}=\left(2 \bar{u} \gamma^{\mu} u-\bar{d} \gamma^{\mu} d-\bar{s} \gamma^{\mu} s\right) / 3$. The Sachs electric and magnetic form factors are related to the Dirac and Pauli form factors, $F_{1}^{N}$ and $F_{2}^{N}$, through [41] (see also, e.g., [42])

$$
G_{E}^{N}\left(q^{2}\right)=F_{1}^{N}\left(q^{2}\right)+\frac{q^{2}}{4 m_{N}^{2}} F_{2}^{N}\left(q^{2}\right), \quad \text { and } \quad G_{M}^{N}\left(q^{2}\right)=F_{1}^{N}\left(q^{2}\right)+F_{2}^{N}\left(q^{2}\right) .
$$

At zero recoil one has for the electric form factor, $G_{E}^{p}(0)=1, G_{E}^{n}(0)=0$, while the magnetic form factor at zero recoil gives [35],

$$
G_{M}^{p}(0)=\mu_{p} \simeq 2.793, \quad G_{M}^{n}(0)=\mu_{n} \simeq-1.913,
$$

i.e., the proton and neutron magnetic moments in units of nuclear magnetons $\hat{\mu}_{N}=$ $e /\left(2 m_{N}\right)$. The anomalous magnetic moments are $F_{2}^{p}(0)=a_{p}, F_{2}^{n}(0)=a_{n}$. The charge radii of the proton and neutron are defined through

$$
G_{E}^{N}\left(q^{2}\right)=G_{E}^{N}(0)+\frac{1}{6}\left[r_{E}^{N}\right]^{2} q^{2}+\cdots
$$

\section{A.2 Axial vector current}

The matrix element of the axial-vector current (2.3) is parametrized by two sets of form factors, $F_{A}^{q / N}\left(q^{2}\right)$ and $F_{P^{\prime}}^{q / N}\left(q^{2}\right)$. For the LO expressions we only need $F_{A}^{q / N}(0)$ and the light meson pole parts of $F_{P^{\prime}}^{q / N}\left(q^{2}\right)$,

$$
F_{P^{\prime}}^{q / N}\left(q^{2}\right)=\frac{m_{N}^{2}}{m_{\pi}^{2}-q^{2}} a_{P^{\prime}, \pi}^{q / N}+\frac{m_{N}^{2}}{m_{\eta}^{2}-q^{2}} a_{P^{\prime}, \eta}^{q / N}+\cdots
$$


The axial vector form factors $F_{A}^{q / N}$ at zero momentum transfer are obtained from the matrix elements $2 m_{p} s^{\mu} \Delta q_{p}=\left\langle p\left|\bar{q} \gamma^{\mu} \gamma_{5} q\right| p\right\rangle_{Q}$, where $|p\rangle$ and $\langle p|$ denote proton states at rest. Moreover, $s^{\mu}$ is the proton's polarization vector such that $s^{2}=-1, s \cdot k_{p}=0$, where $k_{p}^{\mu}=m_{p}(1,0,0,0)$ is the proton four-momentum, and the matrix element is evaluated at scale $Q$. Consequently we find

$$
F_{A}^{q / p}(0)=\Delta q_{p},
$$

while for the residua of the pion- and eta-pole contributions to $F_{P^{\prime}}^{q / N}$ we have

$$
\begin{aligned}
& a_{P^{\prime}, \pi}^{u / p}=-a_{P^{\prime}, \pi}^{d / p}=2 g_{A}, \quad a_{P^{\prime}, \pi}^{s / p}=0, \\
& a_{P^{\prime}, \eta}^{u / p}=a_{P^{\prime}, \eta}^{d / p}=-\frac{1}{2} a_{P^{\prime}, \eta}^{s / p}=\frac{2}{3}\left(\Delta u_{p}+\Delta d_{p}-2 \Delta s_{p}\right) .
\end{aligned}
$$

As always, the coefficients for the neutrons are obtained through a replacement $p \rightarrow n, u \leftrightarrow$ $d$ (no change is implied for $g_{A}$ ). We work in the isospin limit, so that

$$
\Delta u \equiv \Delta u_{p}=\Delta d_{n}, \quad \Delta d \equiv \Delta d_{p}=\Delta u_{n}, \quad \Delta s \equiv \Delta s_{p}=\Delta s_{n} .
$$

The isovector combination is determined precisely from nuclear $\beta$ decay [35],

$$
\Delta u-\Delta d=g_{A}=1.2723(23) .
$$

In the $\overline{\mathrm{MS}}$ scheme at $Q=2 \mathrm{GeV}$ the averages of lattice QCD results give $\Delta u+\Delta d=$ $0.521(53)$ [43], $\Delta s=-0.031(5)$ (averaging over [44-47] and inflating the errors in [46] by a factor of 2 because no continuum extrapolation was performed). Combining with eq. (A.18) this gives [43]

$$
\Delta u=0.897(27), \quad \Delta d=-0.376(27), \quad \Delta s=-0.031(5),
$$

all at the scale $Q=2 \mathrm{GeV}$. The experiments give $\Delta u=0.843(12), \Delta d=-0.427(12)$ [47], in good agreement with the lattice QCD, and a somewhat larger value for the $s$-quark, $\Delta s=-0.084 \pm 0.017$, averaging over HERMES [48] and COMPASS [49] results (see also axion review in [35]). Note that, while the matrix elements $\Delta q$ are scale dependent, the non-isosinglet combinations $\Delta u-\Delta d$ and $\Delta u+\Delta d-2 \Delta s$ are scale independent, since they are protected by non-anomalous Ward identities.

The derivative of the axial form factor at zero recoil is well known for the $u-d$ current. Using the dipole ansatz [50] gives $F_{A}^{\prime}(0) / F_{A}(0)=2 / m_{A}^{2}$, with $m_{A}$ the appropriate dipole mass. A global average over experimental [51, 52] and lattice [47, 53] gives for the $u-d$ current dipole mass $m_{A}^{u-d}=1.064(29) \mathrm{GeV}$, rescaling the combined error following the PDG prescription (the $z$-expansion analysis leads to larger error estimates, corresponding to $\left.m_{A}^{u-d}=1.01(24) \mathrm{GeV}[50]\right)$, while for the $u+d$ current one has $m_{A}^{u+d}=1.64(14) \mathrm{GeV}[47,54]$ and for the strange-quark current, $m_{A}^{s}=0.82(21) \mathrm{GeV}$ [47]. This gives

$$
F_{A}^{u / p \prime}(0)=1.32(7) \mathrm{GeV}^{-2}, \quad F_{A}^{d / p \prime}(0)=-0.93(7) \mathrm{GeV}^{-2},
$$

or in terms of normalized derivatives

$$
\frac{F_{A}^{u / p \prime}(0)}{F_{A}^{u / p}(0)}=1.47(8) \mathrm{GeV}^{-2}, \quad \frac{F_{A}^{d / p \prime}(0)}{F_{A}^{d / p}(0)}=2.47(22) \mathrm{GeV}^{-2},
$$


while for the strange quark

$$
\frac{F_{A}^{s / p \prime}(0)}{F_{A}^{s / p}(0)}=(3.0 \pm 1.5) \mathrm{GeV}^{-2} .
$$

At NLO $F_{P^{\prime}}^{q / N}\left(q^{2}\right)$ needs to be expanded to

$$
F_{P^{\prime}}^{q / N}\left(q^{2}\right)=\frac{m_{N}^{2}}{m_{\pi}^{2}-q^{2}} a_{P^{\prime}, \pi}^{q / N}+\frac{m_{N}^{2}}{m_{\eta}^{2}-q^{2}} a_{P^{\prime}, \eta}^{q / N}+b_{P^{\prime}}^{q / N}+\cdots
$$

At NLO the residua of the poles change by corrections of $\mathcal{O}\left(m_{\pi, \eta}^{2} /\left(4 \pi f_{\pi}^{2}\right)^{2}\right) \approx 0.01-0.05$. For instance, for the $u-d$ current one has at NLO in HBChPT [55],

$$
F_{P^{\prime}}^{(u-d) / p}=\frac{4 m_{N}^{2}}{m_{\pi}^{2}-q^{2}}\left[g_{A}-\frac{2 m_{\pi}^{2} \tilde{B}_{2}}{\left(4 \pi f_{\pi}\right)^{2}}\right]-\frac{2}{3} g_{A} m_{N}^{2} r_{A}^{2},
$$

where $\tilde{B}_{2} \approx-1.0 \pm 0.5$ is the HBChPT low energy constant, while $r_{A}^{2}=6 F_{A}^{\prime}(0) / F_{A}$. The constant term $b_{P^{\prime}}$ is, therefore, for the $u-d$ current given by

$$
b_{P^{\prime}}^{(u-d) / p}=-4 g_{A} m_{N}^{2} \frac{F_{A}^{(u-d) / p \prime}(0)}{F_{A}^{(u-d) / p}(0)} .
$$

Assuming that the relation (A.25) is valid for each quark flavor separately, i.e., neglecting the anomaly contribution to $b_{P^{\prime}}^{q / p}$, gives

$$
b_{P^{\prime}}^{u / p} \approx-4.65(25), \quad b_{P^{\prime}}^{d / p} \approx 3.28(25), \quad b_{P^{\prime}}^{s / p} \approx(-11 \pm 6) \Delta s .
$$

as well as $b_{P^{\prime}}^{s / p} \approx 0.32(18)$. In our numerical analysis we estimated the importance of NLO corrections by keeping $a_{P^{\prime}, \pi}^{q / N}, a_{P^{\prime}, \eta}^{q / N}$ at their LO values, while setting $b_{P^{\prime}}^{q / N}$ to the values in (A.26). Note that these are a small correction to the LO expression when the pion pole is present, but can be important when this is not the case.

\section{A.3 Scalar current}

The scalar form factors $F_{S}^{q / N}$, eq. (2.4), evaluated at $q^{2}=0$ are conventionally referred to as nuclear sigma terms,

$$
F_{S}^{q / N}(0)=\sigma_{q}^{N}
$$

where $\sigma_{q}^{N} \bar{u}_{N} u_{N}=\left\langle N\left|m_{q} \bar{q} q\right| N\right\rangle,|N\rangle$ and $\langle N|$ represent the nucleon states at rest. Another common notation is $\sigma_{q}^{N}=m_{N} f_{T q}^{N}$. Taking the naive average of the most recent lattice QCD determinations [56-58], we find

$$
\sigma_{s}^{p}=\sigma_{s}^{n}=(41.3 \pm 7.7) \mathrm{MeV} .
$$

The matrix elements of the $u$ and $d$ quarks are related to the pion-nucleon sigma term, defined as $\sigma_{\pi N}=\langle N|\bar{m}(\bar{u} u+\bar{d} d)| N\rangle$, where $\bar{m}=\left(m_{u}+m_{d}\right) / 2$. A Heavy Baryon Chiral Perturbation Theory analysis of the $\pi N$ scattering data gives $\sigma_{\pi N}=59(7) \mathrm{MeV}$ [59], and a fit of $\pi N$ scattering data to a representation based on Roy-Steiner equations gives 
$\sigma_{\pi N}=58(5) \mathrm{MeV}$ [60]. A more precise determination is obtained from pionic atoms, $\sigma_{\pi N}=(59.1 \pm 3.5) \mathrm{MeV}$ [61]. These are in agreement with $\sigma_{\pi N}=52(3)(8) \mathrm{MeV}$ obtained from a fit to world lattice $N_{f}=2+1 \mathrm{QCD}$ data at the time [62]. Including, however, both $\Delta(1232)$ and finite spacing in the fit shifted the central value to $\sigma_{\pi N}=44 \mathrm{MeV}$. More recent lattice QCD determinations prefer an even slightly lower value, $\sigma_{\pi N}=38(2) \mathrm{MeV}$ (the average of results in $[57,58,63]$, see also remarks in [64]). We thus use a rather conservative estimate $\sigma_{\pi N}=(50 \pm 15) \mathrm{MeV}$. Using the expressions in [65] this gives

$$
\begin{array}{rlrl}
\sigma_{u}^{p} & =(17 \pm 5) \mathrm{MeV}, & & \sigma_{d}^{p}=(32 \pm 10) \mathrm{MeV}, \\
\sigma_{u}^{n}=(15 \pm 5) \mathrm{MeV}, & \sigma_{d}^{n}=(36 \pm 10) \mathrm{MeV} .
\end{array}
$$

For corrections of higher order in chiral counting one would also need $F_{S}^{q / N}(0)$. These are of the same order, $\mathcal{O}(q)$, as the two-nucleon contributions which are not captured in our expressions.

\section{A.4 Pseudoscalar current}

In the LO expressions we only need the light meson pole parts of the pseudoscalar form factor, eq. (2.5),

$$
F_{P}^{q / N}\left(q^{2}\right)=\frac{m_{N}^{2}}{m_{\pi}^{2}-q^{2}} a_{P, \pi}^{q / N}+\frac{m_{N}^{2}}{m_{\eta}^{2}-q^{2}} a_{P, \eta}^{q / N}+\cdots
$$

The residua of the poles are given by

$$
\begin{aligned}
& \frac{a_{P, \pi}^{u / p}}{m_{u}}=-\frac{a_{P, \pi}^{d / p}}{m_{d}}=\frac{B_{0}}{m_{N}} g_{A}, \quad \frac{a_{P, \pi}^{s / p}}{m_{s}}=0, \\
& \frac{a_{P, \eta}^{u / p}}{m_{u}}=\frac{a_{P, \eta}^{d / p}}{m_{d}}=-\frac{1}{2} \frac{a_{P, \eta}^{s / p}}{m_{s}}=\frac{B_{0}}{3 m_{N}}\left(\Delta u_{p}+\Delta d_{p}-2 \Delta s_{p}\right),
\end{aligned}
$$

where the values of the axial-vector elements, $\Delta q$, are given in (A.18) and (A.19). Moreover, $B_{0}$ is a ChPT constant related to the quark condensate given, up to corrections of $\mathcal{O}\left(m_{q}\right)$, by $\langle\bar{q} q\rangle \simeq-f^{2} B_{0}$. Using quark condensate from [66] and the LO relation $f=f_{\pi}$, with $f_{\pi}$ the pion decay constant, one has $B_{0}=2.666(57) \mathrm{GeV}$, evaluated at the scale $\mu=2 \mathrm{GeV}$.

In practice, $B_{0}$ never appears by itself, but rather as the product $B_{0} m_{q}$ which can be expressed in terms of the pion mass and quark mass ratios,

$$
\begin{aligned}
B_{0} m_{u} & =\frac{m_{\pi}^{2}}{1+m_{d} / m_{u}}=(6.1 \pm 0.5) \times 10^{-3} \mathrm{GeV}^{2}, \\
B_{0} m_{d} & =\frac{m_{\pi}^{2}}{1+m_{u} / m_{d}}=(13.3 \pm 0.5) \times 10^{-3} \mathrm{GeV}^{2}, \\
B_{0} m_{s} & =\frac{m_{\pi}^{2}}{2} \frac{m_{s}}{\bar{m}}=(268 \pm 3) \times 10^{-3} \mathrm{GeV}^{2} .
\end{aligned}
$$

The numerical values are obtained using the ratios of quark masses, $m_{u} / m_{d}=0.46 \pm 0.05$, $m_{s} / \bar{m}=27.5 \pm 0.3$ (see the quark mass review in [35]), and the charged-pion mass $m_{\pi}$. 
At NLO in the chiral expansion, the above expressions for $a_{P, \pi}^{q / p}$ and $a_{P, \eta}^{q / p}$ get corrections of $\mathcal{O}\left(m_{\pi, \eta}^{2} /\left(4 \pi f_{\pi}\right)^{2}\right)$. In addition one needs to keep the constant term in the $q^{2}$ expansion of the form factor

$$
F_{P}^{q / N}\left(q^{2}\right)=\frac{m_{N}^{2}}{m_{\pi}^{2}-q^{2}} a_{P, \pi}^{q / N}+\frac{m_{N}^{2}}{m_{\eta}^{2}-q^{2}} a_{P, \eta}^{q / N}+b_{P}^{q / N}+\cdots
$$

In our numerical analysis we estimate the size of these higher-order corrections by using the NDA size for

$$
b_{P}^{q / N} \approx 1, \quad \text { where } \quad q=u, d, s,
$$

while keeping $a_{P, \pi}^{q / p}, a_{P, \eta}^{q / p}$ at their LO values. This treatment of NLO corrections is only approximate, but suffices for the present precision. Furthermore, it can be improved in the future.

\section{A.5 CP-even gluonic current}

The matrix element of the CP-even gluonic current (2.6) is parametrized by a single form factor $F_{G}^{N}\left(q^{2}\right)$. The LO expressions in chiral counting require only its value at zero momentum transfer,

$$
F_{G}^{N}(0)=-\frac{2 m_{G}}{27}
$$

The nonperturbative coefficient $m_{G}$ is the gluonic contribution to the nucleon mass in the isospin limit,

$$
m_{G} \bar{u}_{N} u_{N}=-\frac{9 \alpha_{s}}{8 \pi}\left\langle N\left|G_{\mu \nu} G^{\mu \nu}\right| N\right\rangle .
$$

The trace of the stress-energy tensor, $\theta_{\mu}^{\mu}=-9 \alpha_{s} /(8 \pi) G_{\mu \nu} G^{\mu \nu}+\sum_{u, d, s} m_{q} \bar{q} q$, yields the relation

$$
m_{G}=m_{N}-\sum_{q} \sigma_{q}^{N}=(848 \pm 14) \mathrm{MeV}
$$

where in the last equality we used the values for $\sigma_{q}$ in (A.28) and (A.29). While the isospin violation in the $\sigma_{q}^{N}$ values is of $\mathcal{O}(10 \%)$, this translates to a very small isospin violation in $m_{G}$, of less than $1 \mathrm{MeV}$. The value of $m_{G}$ in (A.38) thus applies to both $N=p$ and $N=n$.

For the derivative of $F_{G}$ at zero recoil we use the naive dimensional analysis estimate

$$
\frac{F_{G}^{\prime}(0)}{F_{G}(0)} \approx 1 / m_{N}^{2} \approx 1 \mathrm{GeV}^{-2}
$$

\section{A.6 CP-odd gluonic current}

The matrix element of the CP-odd gluonic current (2.7) is related to the matrix elements of the axial and pseudoscalar currents through the QCD chiral anomaly. Namely, a chiral rotation of the quark fields, $q \rightarrow \exp \left(i \beta \gamma_{5}\right) q$, shifts the QCD theta spurion by $\theta \rightarrow \theta-2 \operatorname{Tr} \beta$, along with corresponding changes in the pseudoscalar and axial-vector spurions (see ref. [1]). This implies a relation,

$$
\frac{1}{\tilde{m}}\left\langle N^{\prime}\left|\frac{\alpha_{s}}{8 \pi} G_{\mu \nu}^{a} \tilde{G}^{a \mu \nu}\right| N\right\rangle=\sum_{q}\left(\left\langle N^{\prime}\left|\bar{q} i \gamma_{5} q\right| N\right\rangle-\frac{1}{2 m_{q}} \partial_{\mu}\left\langle N^{\prime}\left|\bar{q} \gamma^{\mu} \gamma_{5} q\right| N\right\rangle\right),
$$


valid at leading order in the chiral expansion. To shorten the notation we defined $1 / \tilde{m}=$ $\left(1 / m_{u}+1 / m_{d}+1 / m_{s}\right)$. In terms of form factors this gives

$$
\frac{1}{\tilde{m}} F_{\tilde{G}}^{N}=\sum_{q}\left(\frac{1}{m_{q}} F_{P}^{q / N}-\frac{m_{N}}{m_{q}} F_{A}^{q / N}-\frac{q^{2}}{4 m_{N} m_{q}} F_{P^{\prime}}^{q / N}\right) .
$$

The leading order contributions from $F_{P}^{q / N}$ cancel in the sum, giving

$$
\begin{aligned}
F_{\tilde{G}}^{N}\left(q^{2}\right)= & -\tilde{m} m_{N}\left[\frac{\Delta u}{m_{u}}+\frac{\Delta d}{m_{d}}+\frac{\Delta s}{m_{s}}+\frac{g_{A}}{2}\left(\frac{1}{m_{u}}-\frac{1}{m_{d}}\right) \frac{q^{2}}{m_{\pi}^{2}-q^{2}}\right. \\
& \left.+\frac{1}{6}(\Delta u+\Delta d-2 \Delta s)\left(\frac{1}{m_{u}}+\frac{1}{m_{d}}-\frac{2}{m_{s}}\right) \frac{q^{2}}{m_{\eta}^{2}-q^{2}}\right] .
\end{aligned}
$$

The pion pole contribution would vanish in the exact isospin limit. However, the isospin breaking effects in the matrix element of $\tilde{G} G$ operator are not small [36]. This is unlike most of the other observables, where isospin breaking is suppressed by the chiral scale, $\propto\left(m_{u}-m_{d}\right) /\left(4 \pi f_{\pi}\right)$. Here, the isospin breaking is proportional to $\left(m_{u}-m_{d}\right) /\left(m_{u}+m_{d}\right) \sim$ $\mathcal{O}(1)$ and is thus large. Similarly, the $\eta$ pole contribution would vanish in the limit of exact $\mathrm{SU}(3)$, but is in fact an $\mathcal{O}(1)$ correction.

The LO expression for $F_{\tilde{G}}^{N}$, eq. (A.42), contains both the constant term as well as poles of the form $\sim q^{2} /\left(m_{\pi}^{2}-q^{2}\right)$. At NLO in chiral counting one also has in addition the $\mathcal{O}\left(q^{2}\right)$ contribution,

$$
F_{\tilde{G}}^{N}\left(q^{2}\right)=\frac{q^{2}}{m_{\pi}^{2}-q^{2}} a_{\tilde{G}, \pi}^{N}+\frac{q^{2}}{m_{\eta}^{2}-q^{2}} a_{\tilde{G}, \eta}^{N}+b_{\tilde{G}}^{N}+c_{\tilde{G}}^{N} q^{2}+\cdots
$$

At NLO the $a_{\tilde{G}, \pi}^{N}, a_{\tilde{G}, \eta}^{N}, b_{\tilde{G}}^{N}$ coefficients differ from their LO values in (A.42) by relative correction of the size $\mathcal{O}\left(m_{\pi, \eta}^{2} /\left(4 \pi f_{\pi}\right)^{2}\right)$, while the NDA estimate for the NLO coefficient is $c_{\tilde{G}}^{N} \approx 1$.

\section{A.7 Tensor current}

The matrix element of the tensor current (2.8) is described by three form factors, $F_{T, 0}^{q / N}\left(q^{2}\right)$, $F_{T, 1}^{q / N}\left(q^{2}\right), F_{T, 2}^{q / N}\left(q^{2}\right)$. These are related to the generalized tensor form factors through (see, e.g., $[67,68])$

$$
\begin{aligned}
& F_{T, 0}^{q / N}\left(q^{2}\right)=m_{q} A_{T, 10}^{q / N}\left(q^{2}\right), \\
& F_{T, 1}^{q / N}\left(q^{2}\right)=-m_{q} B_{T, 10}^{q / N}\left(q^{2}\right), \\
& F_{T, 2}^{q / N}\left(q^{2}\right)=\frac{m_{q}}{2} \tilde{A}_{T, 10}^{q / N}\left(q^{2}\right) .
\end{aligned}
$$

In the LO expressions for DM scattering only $F_{T, 0}^{q / N}(0)$ and $F_{T, 1}^{q / N}(0)$ appear. The value of $F_{T, 0}^{q / N}(0)$ is quite well determined. A common notation is $A_{T, 10}^{q / p}(0)=g_{T}^{q}$ (with $A_{T, 10}^{u(d) / p}=A_{T, 10}^{d(u) / n}$ and $A_{T, 10}^{s / p}=A_{T, 10}^{s / n}$ in the isospin limit), so that

$$
F_{T, 0}^{q / p}(0)=m_{q} g_{T}^{q}
$$


The tensor charges are related to the transversity structure functions $\delta q_{N}(x, \mu)$ by $g_{T}^{q}(\mu)=$ $\int_{-1}^{1} d x \delta q_{N}(x, \mu)$. These structure functions can, in principle, be measured in deep inelastic scattering, but this determination is not very precise. Recent lattice calculations include both connected and disconnected contributions and give, in the $\overline{\mathrm{MS}}$ scheme at $\mu=2 \mathrm{GeV}[69,70]$,

$$
g_{T}^{u}=0.794 \pm 0.015, \quad g_{T}^{d}=-0.204 \pm 0.008, \quad g_{T}^{s}=(3.2 \pm 8.6) \cdot 10^{-4} .
$$

This agrees well with previous, less precise, determinations [67, 71-77]. It is interesting to compare (A.48) with the results from the constituent quark model [78], $g_{T}^{u}=0.97$, $g_{T}^{d}=-0.24$, as we will have to use this model below. In the nonrelativistic quark model, on the other hand, using just $\mathrm{SU}(6)$ spin-flavor symmetry, one gets $g_{T}^{u}=4 / 3, g_{T}^{d}=-1 / 3$, see, e.g., [79].

The zero recoil values of the other two form factors, $F_{T, 1}^{q / N}(0)$ and $F_{T, 2}^{q / N}(0)$, are less well determined. The constituent quark model of [78] gives

$$
\begin{aligned}
B_{T, 10}^{u / p}(0) \approx 3.0, & \tilde{A}_{T, 10}^{u / p} \approx-0.50, \\
B_{T, 10}^{d / p}(0) \approx 0.24, & \tilde{A}_{T, 10}^{d / p} \approx 0.46 .
\end{aligned}
$$

The form factors for the neutron are obtained through the replacements $u \leftrightarrow d, p \rightarrow n$. We assign a $50 \%$ error to the above estimates, taking as a guide twice the difference between the determination of $g_{T}^{q}$ in this model and in lattice QCD (A.48). For the $s$ quark we use the very rough estimates

$$
-0.2 \lesssim B_{T, 10}^{s / p}(0), \tilde{A}_{T, 10}^{s / p}(0) \lesssim 0.2
$$

The linear combination

$$
\kappa_{T}^{q}=2 \tilde{A}_{T, 10}^{q / p}(0)+B_{T, 10}^{q / p}(0)
$$

is in fact much better known than $\tilde{A}_{T, 10}(0)$ and $B_{T, 10}(0)$ separately. The tensor magnetic moments, $\kappa_{T}^{q}$, for the $u$ and $d$ quarks were determined using lattice QCD to be, at $\mu=$ $2 \mathrm{GeV}[80]$,

$$
\kappa_{T}^{u} \approx 3.0, \quad \kappa_{T}^{d} \approx 1.9
$$

(no uncertainty is given in this reference). In the constituent quark model of [78] one gets $\kappa_{T}^{u} \approx 2.0, \kappa_{T}^{d} \approx 1.2$, which agrees with (A.53) within the assigned $50 \%$ uncertainty (larger values $\kappa_{T}^{u}=3.60, \kappa_{T}^{d}=2.36$ are obtained with a simple harmonic oscillator wave function $[78,81])$. For the strange quark one obtains from the $\mathrm{SU}(3)$ chiral quark-soliton model [82]

$$
-0.2 \lesssim \kappa_{T}^{s} \lesssim 0.2
$$

motivating the ranges in (A.51) (in [83] a much smaller value $\kappa_{T}^{s} \approx 0.01$ was found.

In refs. [67, 76, 84], lattice QCD results for the $q^{2}$ dependence of $F_{T, 0}^{q / N}$ for $u$ and $d$ quarks were presented. Averaging over them gives

$$
\frac{F_{T, 0}^{u / p \prime}(0)}{F_{T, 0}^{u / p}(0)} \approx(0.8 \pm 0.3) \mathrm{GeV}^{-2}, \quad \frac{F_{T, 0}^{d / p \prime}(0)}{F_{T, 0}^{d / p}(0)} \approx(0.7 \pm 0.2) \mathrm{GeV}^{-2}
$$


where the errors reflect the differences between the three determinations. For the $s$-quark form factor one can use the NDA estimate, $F_{T, 0}^{s / p_{\prime}}(0) / F_{T, 0}^{s / p}(0) \approx 1 \mathrm{GeV}^{-2}$, consistent with the above.

For the other two form factors an estimate of the derivative at zero recoil can be made using the results from the constituent quark model of [78], giving

$$
\begin{array}{ll}
\frac{F_{T, 1}^{u / p_{\prime}}(0)}{F_{T, 1}^{u / p}(0)} \approx 1.0 \mathrm{GeV}^{-2}, & \frac{F_{T, 1}^{d / p_{\prime}}(0)}{F_{T, 1}^{d / p}(0)} \approx-0.1 \mathrm{GeV}^{-2}, \\
\frac{F_{T, 2}^{u / p_{\prime}}(0)}{F_{T, 2}^{u / p}(0)} \approx 1.2 \mathrm{GeV}^{-2}, & \frac{F_{T, 2}^{d / p_{\prime}}(0)}{F_{T, 2}^{d / p}(0)} \approx 1.0 \mathrm{GeV}^{-2} .
\end{array}
$$

These estimates most probably have large errors, since within this model one gets $F_{T, 0}^{u / p_{\prime}}(0) / F_{T, 0}^{u / p}(0) \approx 0.22 \mathrm{GeV}^{-2}, F_{T, 0}^{d / p_{\prime}}(0) / F_{T, 0}^{d / p}(0) \approx 0.24 \mathrm{GeV}^{-2}$, about a factor of three smaller than lattice QCD determination in (A.55). For the strange quark form factor we vary the derivative at zero recoil in the range

$$
-2 \mathrm{GeV}^{-2} \lesssim F_{T, 1}^{s / p \prime}(0), F_{T, 2}^{s / p \prime}(0) \lesssim 2 \mathrm{GeV}^{-2}
$$

motivated by the slope $d \kappa_{T}^{s} / d q^{2} \approx-2.2 \mathrm{GeV}^{-2}$ that one can deduce from the results in [83].

\section{B Nonrelativistic expansion of currents for fermions}

In this appendix we give the nonrelativistic expansion of the DM and nucleon currents. We first focus on fermionic DM and then translate the results to nonrelativistic nucleons. In order to get rid of the time derivative, $v \cdot \partial$, in the higher-order terms in the Heavy Dark Matter Effective Theory (HDMET) Lagrangian, the tree level relation

$$
\chi=e^{-i m_{\chi} v \cdot x}\left(1+\frac{i \not_{\perp}}{i v \cdot \partial+2 m_{\chi}-i \epsilon}\right) \chi_{v},
$$

is supplemented with a field redefinition ${ }^{5}[86]$

$$
\chi_{v} \rightarrow\left(1-\frac{\partial_{\perp}^{2}}{8 m_{\chi}^{2}}+\frac{\partial_{\perp}^{2}(i v \cdot \partial)}{16 m_{\chi}^{3}}+\cdots\right) \chi_{v}
$$

where $\partial_{\perp}^{\mu}=\partial^{\mu}-v \cdot \partial v^{\mu}$. In this way one obtains the conventional "NRQED" Lagrangian,

$$
\mathcal{L}_{\mathrm{NRQED}}=\chi_{v}^{\dagger}\left(i v \cdot \partial+\frac{\left(i \partial_{\perp}\right)^{2}}{2 m_{\chi}}+\frac{\left(i \partial_{\perp}\right)^{4}}{8 m_{\chi}^{3}}+\cdots\right) \chi_{v}
$$

also beyond $\mathcal{O}\left(p^{2}\right)$ order.

\footnotetext{
${ }^{5}$ In order for the scattering rates to be independent of this arbitrary field redefinition, contributions to the scattering amplitude from the time-ordered product of the Lagrangians (1.10) and (B.3) have to be included [85]. An explicit calculation shows that, with our choice (B.2), these additional contributions vanish to $\mathcal{O}\left(p^{2}\right)$.
} 
Using (B.1) together with (B.2) and applying the equation of motion derived from eq. (B.3) we obtain for the DM currents

$$
\begin{aligned}
& \bar{\chi} \chi \rightarrow \bar{\chi}_{v} \chi_{v}+\frac{i}{2 m_{\chi}^{2}} \epsilon_{\alpha \beta \mu \nu} v^{\alpha}\left(\bar{\chi}_{v} S_{\chi}^{\beta} \overleftarrow{\partial}_{\perp}^{\mu} \vec{\partial}_{\perp}^{\nu} \chi_{v}\right)-\frac{1}{8 m_{\chi}^{2}} \bar{\chi}_{v} \stackrel{\leftrightarrow}{\partial}_{\perp}^{2} \chi_{v}+\mathcal{O}\left(p^{3}\right) \\
& \bar{\chi} i \gamma_{5} \chi \rightarrow \frac{1}{m_{\chi}} \partial_{\mu}\left(\bar{\chi}_{v} S_{\chi}^{\mu} \chi_{v}\right) \\
& -\frac{1}{4 m_{\chi}^{3}} \partial_{\perp}^{\mu} \bar{\chi}_{v} S_{\chi, \mu}\left(\overleftarrow{\partial}_{\perp}^{2}+\vec{\partial}_{\perp}^{2}\right) \chi_{v}+\frac{1}{8 m_{\chi}^{3}} \chi_{v} S_{\chi} \stackrel{\leftrightarrow}{\partial} \perp\left(\overleftarrow{\partial}_{\perp}^{2}-\vec{\partial}_{\perp}^{2}\right) \chi_{v}+\mathcal{O}\left(p^{4}\right) \\
& \bar{\chi} \gamma^{\mu} \chi \rightarrow v^{\mu} \bar{\chi}_{v} \chi_{v}+\frac{1}{2 m_{\chi}} \bar{\chi}_{v} i \stackrel{\leftrightarrow}{\partial}_{\perp}^{\mu} \chi_{v}+\frac{1}{2 m_{\chi}} \partial_{\nu}\left(\bar{\chi}_{v} \sigma_{\perp}^{\mu \nu} \chi_{v}\right) \\
& +\frac{i}{4 m_{\chi}^{2}} v^{\mu} \bar{\chi}_{v} \overleftarrow{\partial}_{\rho} \sigma_{\perp}^{\rho \nu} \vec{\partial}_{\nu} \chi_{v}-\frac{v^{\mu}}{8 m_{\chi}^{2}} \partial_{\perp}^{2} \bar{\chi}_{v} \chi_{v} \\
& +\frac{1}{16 m_{\chi}^{3}}\left(i \partial^{\mu}\left(\bar{\chi}_{v}\left(\overleftarrow{\partial}_{\perp}^{2}-\vec{\partial}_{\perp}^{2}\right) \chi_{v}\right)-2 \bar{\chi}_{v}\left(\overleftarrow{\partial}_{\perp}^{2}+\vec{\partial}_{\perp}^{2}\right) i \stackrel{\leftrightarrow}{\partial}^{\mu} \chi_{v}\right. \\
& \left.-\bar{\chi}_{v}\left(\vec{\partial}_{\perp}^{2}-\overleftarrow{\partial}_{\perp}^{2}\right) \sigma_{\perp}^{\mu \nu} \stackrel{\leftrightarrow}{\partial}_{\perp}^{\nu} \chi_{v}-2 \partial^{\nu}\left(\bar{\chi}_{v}\left(\vec{\partial}_{\perp}^{2}+\overleftarrow{\partial}_{\perp}^{2}\right) \sigma_{\perp}^{\mu \nu} \chi_{v}\right)\right)+\mathcal{O}\left(p^{4}\right) \\
& \bar{\chi} \gamma^{\mu} \gamma_{5} \chi \rightarrow 2 \bar{\chi}_{v} S_{\chi}^{\mu} \chi_{v}-\frac{i}{m_{\chi}} v^{\mu} \bar{\chi}_{v} S_{\chi} \cdot \stackrel{\leftrightarrow}{\partial} \chi_{v} \\
& -\frac{1}{4 m_{\chi}^{2}} \bar{\chi}_{v} \stackrel{\leftrightarrow}{\partial}_{\perp}^{2} S_{\chi}^{\mu} \chi_{v}-\frac{1}{2 m_{\chi}^{2}} \bar{\chi}_{v}\left(\overleftarrow{\partial}_{\perp}^{\mu} S \cdot \partial_{\perp}+\overleftarrow{\partial}_{\perp} \cdot S \partial_{\perp}^{\mu}\right) \chi_{v} \\
& +\frac{i}{4 m_{\chi}^{2}} \varepsilon^{\mu \nu \alpha \beta} v_{\nu} \bar{\chi}_{v} \overleftarrow{\partial}_{\perp \alpha} \partial_{\perp \beta} \chi_{v}-\frac{i}{8 m_{\chi}^{3}} v^{\mu} \partial_{\nu} \bar{\chi}_{v}\left(\overleftarrow{\partial}_{\perp}^{2}-\vec{\partial}_{\perp}^{2}\right) S_{\chi}^{\nu} \chi_{v} \\
& +\frac{i}{4 m_{\chi}^{3}} v^{\mu} \bar{\chi}_{v}\left(\overleftarrow{\partial}_{\perp}^{2}+\vec{\partial}_{\perp}^{2}\right) \stackrel{\leftrightarrow}{\partial} \cdot S_{\chi} \chi_{v}+\mathcal{O}\left(p^{4}\right) \\
& \bar{\chi} \sigma^{\mu \nu} \chi \rightarrow \bar{\chi}_{v} \sigma_{\perp}^{\mu \nu} \chi_{v}+\frac{1}{2 m_{\chi}}\left(\bar{\chi}_{v} i v{ }^{[\mu} \sigma_{\perp}^{\nu] \rho} \stackrel{\leftrightarrow}{\partial}_{\rho} \chi_{v}-v^{[\mu} \partial^{\nu]} \bar{\chi}_{v} \chi_{v}\right) \\
& +\frac{1}{4 m_{\chi}^{2}} \bar{\chi}_{v} \overleftarrow{\partial}_{\perp} \sigma_{\perp}^{\mu \nu} \vec{\partial}_{\perp} \chi_{v}-\frac{1}{8 m_{\chi}^{2}} \bar{\chi}_{v}\left(\overleftarrow{\partial}_{\perp}^{2}+\vec{\partial}_{\perp}^{2}\right) \sigma_{\perp}^{\mu \nu} \chi_{v}+\mathcal{O}\left(p^{3}\right) \\
& \bar{\chi} \sigma^{\mu \nu} i \gamma_{5} \chi \rightarrow 2 \bar{\chi}_{v} S_{\chi}^{[\mu} v^{\nu]} \chi_{v}+\frac{i}{m_{\chi}} \bar{\chi}_{v} S^{[\mu} \stackrel{\leftrightarrow}{\partial}_{\perp}^{\nu]} \chi_{v}+\frac{1}{2 m_{\chi}} \epsilon^{\mu \nu \alpha \beta} v_{\alpha} \partial_{\perp \beta} \bar{\chi}_{v} \chi_{v} \\
& +\frac{1}{4 m_{\chi}^{2}} \partial_{\perp}^{2} \bar{\chi}_{v} v^{[\mu} S_{\chi}^{\nu]} \chi_{v}+\frac{1}{2 m_{\chi}^{2}} \bar{\chi}_{v} \overleftarrow{\partial}_{\perp}^{[\mu} v^{\nu]} S_{\chi} \cdot \vec{\partial}_{\perp} \chi_{v}+\frac{1}{2 m_{\chi}^{2}} \bar{\chi}_{v} \overleftarrow{\partial}_{\perp} \cdot S_{\chi} \vec{\partial}_{\perp}^{[\mu} v^{\nu]} \chi_{v} \\
& +\frac{i}{4 m_{\chi}^{2}} v^{[\mu} \epsilon^{\nu] \alpha \beta \gamma} \bar{\chi}_{v} \overleftarrow{\partial}_{\perp \alpha} \vec{\partial}_{\perp \beta} v_{\gamma} \chi_{v}+\mathcal{O}\left(p^{3}\right)
\end{aligned}
$$

where $\sigma_{\perp}^{\mu \nu}=i\left[\gamma_{\perp}^{\mu}, \gamma_{\perp}^{\nu}\right] / 2, \bar{\chi}_{v} \stackrel{\leftrightarrow}{\partial}^{\mu} \chi_{v}=\bar{\chi}_{v}\left(\partial^{\mu} \chi_{v}\right)-\left(\partial^{\mu} \bar{\chi}_{v}\right) \chi_{v}$, and $S^{\mu}=\gamma_{\perp}^{\mu} \gamma_{5} / 2$ is the spin operator. The square brackets in the last line denote antisymmetrization in the enclosed indices, while the ellipses denote higher orders in $1 / m_{\chi}$. We also used the relation

$$
\bar{\chi}_{v} \sigma_{\perp}^{\mu \nu} \chi_{v}=-2 \epsilon^{\mu \nu \alpha \beta} v_{\alpha}\left(\bar{\chi}_{v} S_{\chi, \beta} \chi_{v}\right)
$$

where $\epsilon^{\mu \nu \alpha \beta}$ is the totally antisymmetric Levi-Civita tensor, with $\epsilon^{0123}=1$, and

$$
\bar{\chi}_{v} S^{\mu} \cdot S^{\nu} \chi_{v}=-\frac{i}{2} \epsilon^{\mu \nu \alpha \beta} \bar{\chi}_{v} v_{\alpha} S_{\beta} \chi_{v}-\frac{1}{4} \bar{\chi}_{v} g_{\perp}^{\mu \nu} \chi_{v} .
$$


The same expressions apply also for nucleon currents, with the obvious replacement $\chi \rightarrow N$. In terms of the momenta instead of derivatives the expansions are

$$
\begin{aligned}
& \bar{\chi} \chi \rightarrow \bar{\chi}_{v} \chi_{v}\left(1+\frac{p_{12}^{2}}{8 m_{\chi}^{2}}\right)+\frac{i}{2 m_{\chi}^{2}} \epsilon_{\alpha \mu \nu \beta} v^{\alpha} p_{2}^{\mu} p_{1}^{\nu}\left(\bar{\chi}_{v} S_{\chi}^{\beta} \chi_{v}\right)+\mathcal{O}\left(p^{3}\right) \\
& \bar{\chi} i \gamma_{5} \chi \rightarrow \frac{-i}{m_{\chi}}\left(\bar{\chi}_{v} q \cdot S_{\chi} \chi_{v}\right)\left(1+\frac{p_{1}^{2}+p_{2}^{2}}{4 m_{\chi}^{2}}\right) \\
& +\frac{i}{8 m_{\chi}^{3}}\left(p_{2}^{2}-p_{1}^{2}\right) \bar{\chi}_{v}\left(S_{\chi} \cdot p_{12}\right) \chi_{v}+\mathcal{O}\left(p^{4}\right), \\
& \bar{\chi} \gamma^{\mu} \chi \rightarrow \bar{\chi}_{v} \chi_{v}\left(v^{\mu}+\frac{p_{12, \perp}^{\mu}}{2 m_{\chi}}+v^{\mu} \frac{q_{\perp}^{2}}{8 m_{\chi}^{2}}\right)+\frac{i}{m_{\chi}} \epsilon^{\alpha \mu \nu \beta} v_{\alpha} q_{\nu}\left(\bar{\chi}_{v} S_{\chi, \beta} \chi_{v}\right) \\
& -\frac{i}{2 m_{\chi}^{2}} v^{\mu} \epsilon^{\alpha \rho \nu \beta} v_{\alpha} p_{2 \rho} p_{1 \nu}\left(\bar{\chi}_{v} S_{\chi, \beta} \chi_{v}\right) \\
& +\frac{1}{16 m_{\chi}^{3}}\left[q^{\mu}\left(p_{1 \perp}^{2}-p_{2 \perp}^{2}\right)+2 p_{12}^{\mu}\left(p_{1 \perp}^{2}+p_{2 \perp}^{2}\right)\right] \bar{\chi}_{v} \chi_{v} \\
& +\frac{i}{8 m_{\chi}^{3}}\left[p_{12, \nu}\left(p_{1 \perp}^{2}-p_{2 \perp}^{2}\right)+2 q_{\nu}\left(p_{1 \perp}^{2}+p_{2 \perp}^{2}\right)\right] \epsilon^{\mu \nu \alpha \beta} v_{\alpha} \bar{\chi}_{v} S_{\chi, \beta} \chi_{v}+\mathcal{O}\left(p^{4}\right) \text {, } \\
& \bar{\chi} \gamma^{\mu} \gamma_{5} \chi \rightarrow 2 \bar{\chi}_{v} S_{\chi}^{\mu} \chi_{v}\left(1+\frac{p_{12 \perp}^{2}}{8 m_{\chi}^{2}}\right)-\frac{1}{m_{\chi}} v^{\mu} \bar{\chi}_{v} S_{\chi} \cdot p_{12} \chi_{v} \\
& -\frac{1}{4 m_{\chi}^{2}} \bar{\chi}_{v}\left(p_{12 \perp}^{\mu} S_{\chi} \cdot p_{12}-q_{\perp}^{\mu} S_{\chi} \cdot q\right) \chi_{v}-\frac{i}{4 m_{\chi}^{2}} \varepsilon^{\nu \mu \alpha \beta} v_{\nu} p_{2 \alpha} p_{1 \beta} \bar{\chi}_{v} \chi_{v} \\
& -\frac{v^{\mu}}{8 m_{\chi}^{3}} \bar{\chi}_{v}\left[\left(p_{1 \perp}^{2}-p_{2 \perp}^{2}\right) q \cdot S_{\chi}+2\left(p_{1 \perp}^{2}+p_{2 \perp}^{2}\right) p_{12} \cdot S_{\chi}\right] \chi_{v}+\mathcal{O}\left(p^{4}\right), \\
& \bar{\chi} \sigma^{\mu \nu} \chi \rightarrow-2 \varepsilon^{\mu \nu \alpha \beta} v_{\alpha}\left(\bar{\chi}_{v} S_{\chi, \beta} \chi_{v}\right)\left(1+\frac{p_{12}^{2}}{8 m_{\chi}^{2}}\right)+\frac{1}{m_{\chi}} v^{[\mu} \varepsilon^{\nu] \delta \alpha \beta} v_{\delta} p_{12, \alpha} \bar{\chi}_{v} S_{\chi, \beta} \chi_{v} \\
& +\frac{i}{2 m_{\chi}} v^{[\mu} q^{\nu]} \bar{\chi}_{v} \chi_{v}+\frac{i}{4 m_{\chi}^{2}} p_{1}^{[\mu} p_{2}^{\nu]} \bar{\chi}_{v} \chi_{v} \\
& +\frac{1}{2 m_{\chi}^{2}} \varepsilon^{\mu \nu \alpha \beta} v_{\alpha} \bar{\chi}_{v}\left(p_{1 \beta} S_{\chi} \cdot p_{2}+p_{2 \beta} S_{\chi} \cdot p_{1}\right) \chi_{v}+\mathcal{O}\left(p^{3}\right), \\
& \bar{\chi} \sigma^{\mu \nu} i \gamma_{5} \chi \rightarrow 2 \bar{\chi}_{v} S_{\chi}^{[\mu} v^{\nu]} \chi_{v}\left(1+\frac{q_{\perp}^{2}}{8 m_{\chi}^{2}}\right)+\frac{1}{m_{\chi}} \bar{\chi}_{v} S_{\chi}^{[\mu} p_{12, \perp}^{\nu]} \chi_{v}-\frac{i}{2 m_{\chi}} \epsilon^{\mu \nu \alpha \beta} v_{\alpha} q_{\beta} \bar{\chi}_{v} \chi_{v} \\
& +\frac{1}{2 m_{\chi}^{2}} \bar{\chi}_{v}\left(p_{1}^{[\mu} v^{\nu]} S_{\chi} \cdot p_{2}+p_{2}^{[\mu} v^{\nu]} S_{\chi} \cdot p_{1}\right) \chi_{v} \\
& -\frac{i}{4 m_{\chi}^{2}} v^{[\mu} \epsilon^{\nu] \delta \alpha \beta} v_{\delta} p_{1 \alpha} p_{2 \beta} \bar{\chi}_{v} \chi_{v}+\mathcal{O}\left(p^{3}\right),
\end{aligned}
$$

where we used the shorthand notation $p_{12}^{\mu}=p_{1}^{\mu}+p_{2}^{\mu}$. The corresponding expansion of the nucleon currents is obtained through the replacements $\chi \rightarrow N, p_{1,2}^{\mu} \rightarrow k_{1,2}^{\mu}, q^{\mu} \rightarrow-q^{\mu}$. 


\section{NLO expressions for fermionic DM}

At NLO in the chiral expansion for the hadronization of the relativistic operators, eqs. (1.3)-(1.9), one encounters terms that are not Galilean invariant, since they depend on the average nucleon velocity,

$$
\vec{v}_{a}=\frac{1}{2 m_{N}}\left(\vec{k}_{1}+\vec{k}_{2}\right)
$$

These terms signal that the underlying theory is, in fact, Lorentz rather than Galilean invariant.

In addition to the nonrelativistic operators (1.11)-(1.19) there are three new operators of $\mathcal{O}(q)$,

$$
\begin{array}{ll}
\mathcal{O}_{1 a}^{N(1)}=\mathbb{1}_{\chi}\left(\vec{v}_{a} \cdot \vec{S}_{N}\right), & \mathcal{O}_{2 a}^{N(1)}=\left(\vec{v}_{a} \cdot \vec{S}_{\chi}\right) \mathbb{1}_{N} \\
\mathcal{O}_{3 a}^{N(1)}=\vec{v}_{a} \cdot\left(\vec{S}_{\chi} \times \vec{S}_{N}\right), &
\end{array}
$$

four new operators of $\mathcal{O}\left(q^{2}\right)$,

$$
\begin{array}{ll}
\mathcal{O}_{1 a}^{N(2)}=\left(\frac{i \vec{q}}{m_{N}} \cdot \vec{S}_{\chi}\right)\left(\vec{v}_{a} \cdot \vec{S}_{N}\right), & \mathcal{O}_{2 a}^{N(2)}=\left(\vec{v}_{a} \cdot \vec{S}_{\chi}\right)\left(\frac{i \vec{q}}{m_{N}} \cdot \vec{S}_{N}\right), \\
\mathcal{O}_{3 a}^{N(2)}=\left(\vec{v}_{a} \cdot \vec{S}_{\chi}\right)\left(\vec{v}_{a} \cdot \vec{S}_{N}\right), & \mathcal{O}_{4 a}^{N(2)}=\left(\frac{i \vec{q}}{m_{N}} \cdot \vec{S}_{\chi}\right)\left(\frac{i \vec{q}}{m_{N}} \cdot \vec{S}_{N}\right),
\end{array}
$$

and three of $\mathcal{O}\left(q^{3}\right)$

$$
\begin{array}{ll}
\mathcal{O}_{1 a}^{N(3)}=\left(\vec{v}_{a} \cdot \vec{S}_{\chi}\right) \vec{v}_{a} \cdot\left(\vec{v}_{\perp} \times \vec{S}_{N}\right), & \mathcal{O}_{2 a}^{N(3)}=\vec{v}_{a} \cdot\left(\vec{v}_{\perp} \times \vec{S}_{\chi}\right)\left(\vec{v}_{a} \cdot \vec{S}_{N}\right), \\
\mathcal{O}_{3 a}^{N(3)}=\left(\frac{i \vec{q}}{m_{N}} \cdot \vec{S}_{N}\right)\left(\frac{i \vec{q}}{m_{N}} \cdot\left(\vec{v}_{a} \times \vec{S}_{\chi}\right)\right) . &
\end{array}
$$

Next we give the expressions for the nonrelativistic reduction of the operators (1.3)-(1.9) to subleading order in $q^{2}$. For each of the operators we stop at the order at which one expects the contributions from the two-nucleon currents. We explicitly include a factor

$$
\sqrt{\frac{E_{p_{1}} E_{p_{2}} E_{k_{1}} E_{k_{2}}}{m_{\chi}^{2} m_{N}^{2}}}=1+\frac{\vec{q}^{2}}{8}\left(\frac{1}{m_{\chi}^{2}}+\frac{1}{m_{N}^{2}}\right)+\frac{1}{2} \vec{v}_{\perp}^{2}+\vec{v}_{a}^{2}+\mathcal{O}\left(\vec{q}^{4}\right)
$$

in order to convert from the usual relativistic normalization of states, $\left\langle\chi\left(p^{\prime}\right) \mid \chi(p)\right\rangle=$ $2 E_{\vec{p}}(2 \pi)^{3} \delta^{3}\left(\vec{p}^{\prime}-\vec{p}\right)$, where $E_{\vec{p}}=\sqrt{\vec{p}^{2}+m_{\chi}^{2}}$, to the normalization used in [5]. The hadronization of the dimension-six interaction operators, including the subleading orders for single- 
nucleon currents, are then given by,

$$
\begin{aligned}
\mathcal{Q}_{1, q}^{(6) \rightarrow} & F_{1}^{q / N} \mathcal{O}_{1}^{N}+\left\{F_{1}^{q / N} \frac{\vec{v}_{\perp}^{2}}{2} \mathcal{O}_{1}^{N}-F_{2}^{q / N} \frac{\vec{q}^{2}}{4 m_{N}^{2}} \mathcal{O}_{1}^{N}-\left(F_{1}^{q / N}+F_{2}^{q / N}\right) \frac{\vec{q}^{2}}{m_{\chi} m_{N}} \mathcal{O}_{4}^{N}\right. \\
& \left.+\left(F_{1}^{q / N}+F_{2}^{q / N}\right) \mathcal{O}_{3}^{N}+\frac{m_{N}}{2 m_{\chi}} F_{1}^{q / N} \mathcal{O}_{5}^{N}+\frac{m_{N}}{m_{\chi}}\left(F_{1}^{q / N}+F_{2}^{q / N}\right) \mathcal{O}_{6}^{N}+\mathcal{O}\left(q^{2}\right)\right\} \\
\mathcal{Q}_{2, q}^{(6)} \rightarrow & 2 F_{1}^{q / N} \mathcal{O}_{8}^{N}+2\left(F_{1}^{q / N}+F_{2}^{q / N}\right) \mathcal{O}_{9}^{N}+\mathcal{O}\left(q^{2}\right) \\
\mathcal{Q}_{3, q}^{(6)} \rightarrow & -2 F_{A}^{q / N}\left(\mathcal{O}_{7}^{N}-\frac{m_{N}}{m_{\chi}} \mathcal{O}_{9}^{N}\right)-\left\{F_{A}^{q / N}\left(\mathcal{O}_{7}^{N}-\frac{m_{N}}{m_{\chi}} \mathcal{O}_{9}^{N}\right) \frac{\vec{q}^{2}}{4 m_{N}^{2}}\right. \\
& \left.-F_{A}^{q / N}\left(\left(\vec{v}_{a} \cdot \vec{v}_{\perp}\right) \mathcal{O}_{1 a}^{N(1)}+\frac{i \vec{q} \cdot \vec{v}_{a}}{m_{\chi}} \mathcal{O}_{3 a}^{N(1)}\right)+\frac{1}{2} F_{P^{\prime}} \frac{i \vec{q} \cdot \vec{v}_{a}}{m_{N}}\left(\vec{v}_{a} \cdot \vec{v}_{\perp}\right) \mathcal{O}_{10}^{N}+\mathcal{O}\left(q^{4}\right)\right\} \\
\mathcal{Q}_{4, q}^{(6)} \rightarrow & -4 F_{A}^{q / N} \mathcal{O}_{4}^{N}+F_{P^{\prime}}^{q / N} \mathcal{O}_{6}^{N}-\left\{\frac{\vec{q}^{2}}{2} F_{A}^{q / N} \mathcal{O}_{4}^{N}\left(\frac{1}{m_{\chi}^{2}}+\frac{1}{m_{N}^{2}}\right)\right. \\
& -\frac{1}{2} F_{A}^{q / N}\left(1+\frac{m_{N}^{2}}{m_{\chi}^{2}}\right) \mathcal{O}_{6}^{N}-\frac{m_{N}}{2 m_{\chi}} F_{A}^{q / N} \mathcal{O}_{3}^{N}+2 F_{A}^{q / N} \mathcal{O}_{2 b}^{N} \\
& \left.-\frac{1}{2} F_{P^{\prime}} \frac{i \vec{q} \cdot \vec{v}_{a}}{m_{N}}\left(\mathcal{O}_{1 a}^{N(2)}+\mathcal{O}_{2 a}^{N(2)}\right)+\mathcal{O}\left(q^{3}\right)\right\}
\end{aligned}
$$

The terms in the curly brackets arise for the first time at subleading order, i.e., at $\mathcal{O}\left(q^{\nu_{\mathrm{LO}}+2}\right)$. The form factors in these expressions are evaluated at $q^{2}=0$, i.e., $F_{i} \rightarrow F_{i}(0)$. In the LO terms, on the other hand, one should expand the form factors to $\mathcal{O}\left(q^{2}\right)$, i.e., in the expressions outside curly brackets, $F_{i} \rightarrow F_{i}(0)+F_{i}^{\prime}(0) q^{2}$.

Note that the hadronization of $\mathcal{Q}_{1, q}^{(6)}$ is expected to receive contributions from twonucleon currents at $\mathcal{O}\left(q^{2}\right)$, i.e., at the same order as the displayed corrections from the single-nucleon current. In the hadronization of $\mathcal{Q}_{2, q}^{(6)}$ we do not show the subleading corrections from expanding the single-nucleon currents. In this case the two-nucleon currents enter at $\mathcal{O}\left(q^{2}\right)$, while the higher-order corrections from single-nucleon currents start only at $\mathcal{O}\left(q^{3}\right)$. Note also that, at $\mathcal{O}\left(p^{4}\right)$, the hadronization of $\mathcal{Q}_{4, q}^{(6)}$ receives a contribution that is coherently enhanced, but suppressed by a numerical factor $\sim 1 /\left(16 m_{N} m_{\chi}\right)$.

The hadronizations of the dimension-seven operators are given by

$$
\begin{aligned}
\mathcal{Q}_{1}^{(7)} \rightarrow & F_{G}^{N} \mathcal{O}_{1}^{N}+\left\{F_{G}^{N} \frac{\vec{q}^{2}}{8}\left(\frac{1}{m_{\chi}^{2}}+\frac{1}{m_{N}^{2}}\right) \mathcal{O}_{1}^{N}-\frac{m_{N}}{2 m_{\chi}} F_{G}^{N} \mathcal{O}_{5}^{N}+\mathcal{O}\left(q^{3}\right)\right\} \\
\mathcal{Q}_{2}^{(7)} \rightarrow & -\frac{m_{N}}{m_{\chi}} F_{G}^{N} \mathcal{O}_{11}^{N}-\left\{\frac{\vec{q}^{2}}{8 m_{N} m_{\chi}} F_{G}^{N} \mathcal{O}_{11}^{N}+\frac{i \vec{q} \cdot \vec{v}_{a}}{m_{\chi}} F_{G}^{N} \mathcal{O}_{2 a}^{N(1)}+\mathcal{O}\left(q^{4}\right)\right\} \\
\mathcal{Q}_{3}^{(7)} \rightarrow & F_{\tilde{G}}^{N} \mathcal{O}_{10}^{N}+\left\{\frac{\vec{q}^{2}}{8 m_{\chi}^{2}} F_{\tilde{G}}^{N} \mathcal{O}_{10}^{N}+\frac{m_{N}}{2 m_{\chi}} F_{\tilde{G}}^{N}\left(\mathcal{O}_{15}^{N}+\frac{\vec{q}^{2}}{m_{N}^{2}} \mathcal{O}_{12}^{N}\right)\right. \\
& \left.+\frac{i \vec{q} \cdot \vec{v}_{a}}{2 m_{N}} F_{\tilde{G}}^{N} \mathcal{O}_{1 a}^{N(1)}+\mathcal{O}\left(q^{4}\right)\right\} \\
\mathcal{Q}_{4}^{(7)} \rightarrow & \frac{m_{N}}{m_{\chi}} F_{\tilde{G}}^{N} \mathcal{O}_{6}^{N}+\left\{\frac{i \vec{q} \cdot \vec{v}_{a}}{2 m_{\chi}} F_{\tilde{G}}^{N}\left(\mathcal{O}_{1 a}^{N(2)}+\mathcal{O}_{2 a}^{N(2)}\right)+\mathcal{O}\left(q^{5}\right)\right\}
\end{aligned}
$$




$$
\begin{aligned}
\mathcal{Q}_{5, q}^{(7)} \rightarrow & F_{S}^{q / N} \mathcal{O}_{1}^{N}+\mathcal{O}(q) \\
\mathcal{Q}_{6, q}^{(7)} \rightarrow & -\frac{m_{N}}{m_{\chi}} F_{S}^{q / N} \mathcal{O}_{11}^{N}+\mathcal{O}\left(q^{2}\right) \\
\mathcal{Q}_{7, q}^{(7)} \rightarrow & F_{P}^{q / N} \mathcal{O}_{10}^{N}+\left\{\frac{\vec{q}^{2}}{8 m_{\chi}^{2}} F_{P}^{q / N} \mathcal{O}_{10}^{N}+\frac{m_{N}}{2 m_{\chi}} F_{P}^{q / N}\left(\mathcal{O}_{15}^{N}+\frac{\vec{q}^{2}}{m_{N}^{2}} \mathcal{O}_{12}^{N}\right)\right. \\
& \left.+\frac{i \vec{q} \cdot \vec{v}_{a}}{2 m_{N}} F_{P}^{N} \mathcal{O}_{1 a}^{N(1)}+\mathcal{O}\left(q^{4}\right)\right\} \\
\mathcal{Q}_{8, q}^{(7)} \rightarrow & \frac{m_{N}}{m_{\chi}} F_{P}^{q / N} \mathcal{O}_{6}^{N}+\left\{\frac{i \vec{q} \cdot \vec{v}_{a}}{2 m_{\chi}} F_{P}^{N}\left(\mathcal{O}_{1 a}^{N(2)}+\mathcal{O}_{2 a}^{N(2)}\right)+\mathcal{O}\left(q^{5}\right)\right\} \\
\mathcal{Q}_{9, q}^{(7)} \rightarrow & 8 F_{T, 0}^{q / N} \mathcal{O}_{4}^{N}+\left\{\left[2 F_{T, 1}^{q / N} \frac{\vec{q}^{2}}{m_{N}^{2}}+F_{T, 0}^{q / N}\left(\frac{\vec{q}^{2}}{m_{\chi}^{2}}+\frac{\vec{q}^{2}}{m_{N}^{2}}-8 \vec{v}_{a}^{2}\right)\right] \mathcal{O}_{4}^{N}+4 F_{T, 0}^{q / N} \mathcal{O}_{2 b}^{N}\right. \\
& -\frac{\vec{q}^{2}}{2 m_{N} m_{\chi}}\left(F_{T, 0}^{q / N}-F_{T, 1}^{q / N}\right) \mathcal{O}_{1}^{N}-\left[\left(1+\frac{m_{N}^{2}}{m_{\chi}^{2}}\right) F_{T, 0}^{q / N}+2 F_{T, 1}^{q / N}\right] \mathcal{O}_{6}^{N} \\
& \left.-\frac{m_{N}}{m_{\chi}} F_{T, 0}^{q / N} \mathcal{O}_{3}^{N}+2\left(F_{T, 0}^{q / N}-F_{T, 1}^{q / N}\right) \mathcal{O}_{5}^{N}+16 F_{T, 0}^{q / N} \mathcal{O}_{3 a}^{N(2)}+\mathcal{O}\left(q^{3}\right)\right\} \\
\mathcal{Q}_{10, q}^{(7)} \rightarrow & -2 \frac{m_{N}}{m_{\chi}} F_{T, 0}^{q / N} \mathcal{O}_{10}^{N}+2\left(F_{T, 0}^{q / N}-F_{T, 1}^{q / N}\right) \mathcal{O}_{11}^{N}-8 F_{T, 0}^{q / N} \mathcal{O}_{12}^{N} \\
& -\left\{\frac{m_{N}}{m_{\chi}} F_{T, 0}^{q / N} \mathcal{O}_{10}^{N}\left(\frac{\vec{q}^{2}}{4 m_{\chi}^{2}}+\vec{v}_{\perp}^{2}\right)+8 F_{T, 0}^{q / N} \mathcal{O}_{12}^{N}\left(\frac{1}{2} \vec{v}_{a}^{2}+\frac{1}{2} \vec{v}_{\perp}^{2}+\frac{\vec{q}^{2}}{8 m_{\chi}^{2}}\right)\right. \\
& +\mathcal{O}_{11}^{N}\left[\left(\frac{\vec{q}^{2}}{4 m_{\chi}^{2}}+\vec{v}_{\perp}^{2}\right) F_{T, 1}^{q / N}-F_{T, 0}^{q / N}\left(3 \vec{v}_{a}^{2}+\vec{v}_{\perp}^{2}+\frac{\vec{q}^{2}}{4 m_{\chi}^{2}}+\frac{\vec{q}^{2}}{4 m_{N}^{2}}\right)\right. \\
& \left.\left.+F_{T, 2}^{q / N}\left(4 \vec{v}_{a}^{2}-\frac{\vec{q}^{2}}{m_{N}^{2}}\right)\right]-\left(2 F_{T, 0}^{q / N}-F_{T, 1}^{q / N}\right) \mathcal{O}_{2 a}^{N(1)}+4 F_{T, 0}^{q / N}\left(\mathcal{O}_{1 a}^{N(3)}+\mathcal{O}_{2 a}^{N(3)}\right)+\mathcal{O}_{T}\left(q^{4}\right)\right\} \\
m_{\chi} & \left.F_{T, 0}^{q / N}\right) \mathcal{O}_{15}^{N}-2 \frac{i \vec{q} \cdot \vec{v}_{a}}{q / N} \mathcal{O}_{1 a}^{N(1)} \\
& \\
& \\
&
\end{aligned}
$$

The expressions that appear for the first time at $\mathcal{O}\left(q^{\nu_{\mathrm{LO}}+2}\right)$ are collected inside the curly brackets. In these the form factors are to be expanded to LO in chiral counting, as denoted in eqs. (2.9)-(2.11). In particular, the form factors without light meson poles are evaluated at $q^{2}=0$, i.e., for these $F_{i} \rightarrow F_{i}(0)$ inside curly brackets. In the terms outside curly brackets, however, the form factors should be expanded to NLO, cf. eqs. (2.9)-(2.11). The operators $\mathcal{Q}_{5, q}^{(7)}$ and $\mathcal{Q}_{6, q}^{(7)}$ receive contributions at $\mathcal{O}\left(q^{\nu_{\mathrm{LO}}+1}\right)$ from two-body currents, so we do not display the corrections from expanding the single-nucleon currents which, in this case, start at $\mathcal{O}\left(q^{\nu_{\mathrm{LO}}+2}\right)$.

\section{Nonrelativistic expansion for scalar DM}

To derive the HDMET for scalar DM, we factor out ${ }^{6}$ the large momenta,

$$
\varphi(x)=e^{-i m_{\varphi} v \cdot x} \varphi_{v},
$$

\footnotetext{
${ }^{6}$ Note that we dropped a global rescaling factor $\left(2 m_{\varphi}\right)^{-1 / 2}$ on the right side of eq. (D.1).
} 
followed by a field redefinition

$$
\varphi_{v} \rightarrow\left(1-i \frac{v \cdot \partial}{4 m_{\varphi}}+\frac{\left(i \partial_{\perp}\right)^{2}}{8 m_{\varphi}^{2}}+\frac{3}{32} \frac{(i v \cdot \partial)^{2}}{m_{\varphi}^{2}}-\frac{3}{32} \frac{(i v \cdot \partial)\left(i \partial_{\perp}\right)^{2}}{m_{\varphi}^{3}}-\frac{5}{128} \frac{(i v \cdot \partial)^{3}}{m_{\varphi}^{3}}+\cdots\right) \varphi_{v} .
$$

This gives the usual HDMET for scalar DM

$$
\mathcal{L}_{\mathrm{HDMET}}=\varphi_{v}^{*} i v \cdot \partial \varphi_{v}+\frac{1}{2 m_{\varphi}} \varphi_{v}^{*}\left(i \partial_{\perp}\right)^{2} \varphi_{v}+\frac{1}{8 m_{\varphi}^{3}} \varphi_{v}^{*}\left(i \partial_{\perp}\right)^{4} \varphi_{v}+\cdots+\mathcal{L}_{\varphi_{v}} .
$$

The first term is the LO HDMET for scalar fields. The $1 / m_{\varphi}$ term is fixed by reparametrization invariance [87], while the ellipses denote the higher-order terms.

The DM bilinears have the following nonrelativistic expansion,

$$
\begin{aligned}
\varphi^{*} \varphi & \rightarrow \varphi_{v}^{*} \varphi_{v}-\frac{1}{4 m_{\varphi}^{2}} \varphi_{v}^{*}\left(\overleftarrow{\partial}^{2}+\vec{\partial}^{2}\right) \varphi_{v}+\mathcal{O}\left(q^{3}\right) \\
i\left(\varphi^{*} \stackrel{\leftrightarrow}{\partial_{\mu}} \varphi\right) & \rightarrow 2 m_{\varphi} v_{\mu}\left(\varphi_{v}^{*} \varphi_{v}\right)+i\left(\varphi_{v}^{*} \stackrel{\leftrightarrow}{\partial}_{\perp, \mu} \varphi_{v}\right)+\mathcal{O}\left(q^{3}\right) \\
\left(\partial^{[\mu} \varphi^{*} \partial^{\nu]} \varphi\right) & \rightarrow i m_{\varphi} v{ }^{[\mu} \partial_{\perp}^{\nu]}\left(\varphi_{v}^{*} \varphi_{v}\right)+\partial_{\perp}^{[\mu} \varphi_{v}^{*} \partial_{\perp}^{\nu]} \varphi_{v}+\frac{i}{4 m_{\varphi}} \varphi_{v}^{*} v^{[\mu} \stackrel{\leftrightarrow}{\partial}_{\perp}^{\nu]}\left(\overleftarrow{\partial}^{2}-\vec{\partial}^{2}\right) \varphi_{v}+\mathcal{O}\left(q^{4}\right)
\end{aligned}
$$

In terms of the momenta these are

$$
\begin{aligned}
\varphi^{*} \varphi & \rightarrow \varphi_{v}^{*} \varphi_{v}\left(1+\frac{p_{1}^{2}+p_{2}^{2}}{4 m_{\varphi}^{2}}\right)+\cdots, \\
i\left(\varphi^{*} \stackrel{\leftrightarrow}{\partial_{\mu}} \varphi\right) & \rightarrow \varphi_{v}^{*} \varphi_{v}\left(2 m_{\varphi} v_{\mu}+p_{12 \perp, \mu}\right)+\cdots \\
\left(\partial^{[\mu} \varphi^{*} \partial^{\nu]} \varphi\right) & \rightarrow m_{\varphi}\left(v^{[\mu} q^{\nu]}+v^{[\mu} p_{12}^{\nu]} \frac{p_{1}^{2}-p_{2}^{2}}{4 m_{\varphi}^{2}}\right) \varphi_{v}^{*} \varphi_{v}+p_{2}^{[\mu} p_{1}^{\nu]} \varphi_{v}^{*} \varphi_{v}+\cdots .
\end{aligned}
$$

The nonrelativistic reductions of the operators describing interactions with scalar DM are thus (again explicitly including a normalization factor similar to (C.8))

$$
\begin{aligned}
\mathcal{Q}_{1 q}^{(6) \rightarrow} & 2 m_{\varphi} F_{1}^{q / N} \mathcal{O}_{1}^{N}\left(1+\frac{\vec{v}_{\perp}^{2}}{2}+\frac{\vec{q}^{2}}{8 m_{\varphi}^{2}}\right)-\frac{\vec{q}^{2}}{2 m_{N}^{2}} m_{\varphi} F_{2}^{q / N} \mathcal{O}_{1}^{N} \\
& +2 m_{\varphi}\left(F_{1}^{q / N}+F_{2}^{q / N}\right) \mathcal{O}_{3}^{N}+\mathcal{O}\left(q^{3}\right) \\
\mathcal{Q}_{2 q}^{(6) \rightarrow} & -4 F_{A}^{q / N} m_{\varphi} \mathcal{O}_{7}^{N}\left[1+\frac{\vec{v}_{a}^{2}}{2}+\frac{\vec{v}_{\perp}^{2}}{2}+\frac{\vec{q}^{2}}{8}\left(\frac{1}{m_{N}^{2}}+\frac{1}{m_{\varphi}^{2}}\right)\right] \\
& -2 F_{A}^{q / N} m_{\varphi}\left(\vec{v}_{a} \cdot \vec{v}_{\perp}\right) \mathcal{O}_{1 a}^{N(1)}+\mathcal{O}\left(q^{4}\right) \\
\mathcal{Q}_{3 q}^{(6)} \rightarrow & F_{S}^{q / N} \mathcal{O}_{1}^{N}\left(1+\frac{\vec{q}^{2}}{8 m_{N}^{2}}\right)+\mathcal{O}\left(q^{4}\right) \\
\mathcal{Q}_{4 q}^{(6)} \rightarrow & F_{P}^{q / N} \mathcal{O}_{10}^{N}+\frac{1}{2} F_{P}^{q / N} \frac{\left(i \vec{q} \cdot \vec{v}_{a}\right)}{m_{N}} \mathcal{O}_{1 a}^{N(1)}+\mathcal{O}\left(q^{4}\right), \\
\mathcal{Q}_{5}^{(6)} \rightarrow & F_{G} \mathcal{O}_{1}^{N}\left(1+\frac{\vec{q}^{2}}{8 m_{N}^{2}}\right)+\mathcal{O}\left(q^{4}\right), \\
\mathcal{Q}_{6}^{(6)} \rightarrow & F_{\tilde{G}} \mathcal{O}_{10}^{N}+\frac{1}{2} F_{\tilde{G}} \frac{\left(i \vec{q} \cdot \vec{v}_{a}\right)}{m_{N}} \mathcal{O}_{1 a}^{N(1)}+\mathcal{O}\left(q^{4}\right),
\end{aligned}
$$

where the non-relativistic operators are defined in eqs. (1.11)-(1.19) and eqs. (C.2)-(C.7). 


\section{E The expressions for the non-relativistic coefficients}

Here we collect the expressions for the coefficients of the non-relativistic operators, eqs. (1.11)-(1.19), in terms of the UV Wilson coefficients, eq. (1.1), and the single-nucleon form factors. We find

$$
\begin{aligned}
c_{1}^{p}= & -\frac{\alpha}{2 \pi m_{\chi}} Q_{p} \hat{\mathcal{C}}_{1}^{(5)}+\sum_{q}\left(F_{1}^{q / p} \hat{\mathcal{C}}_{1, q}^{(6)}+F_{S}^{q / p} \hat{\mathcal{C}}_{5, q}^{(7)}\right)+F_{G}^{p} \hat{\mathcal{C}}_{1}^{(7)} \\
& -\frac{\vec{q}^{2}}{2 m_{\chi} m_{N}} \sum_{q}\left(F_{T, 0}^{q / p}-F_{T, 1}^{q / p}\right) \hat{\mathcal{C}}_{9, q}^{(7)}, \\
c_{4}^{p}= & -\frac{2 \alpha}{\pi} \frac{\mu_{p}}{m_{N}} \hat{\mathcal{C}}_{1}^{(5)}+\sum_{q}\left(8 F_{T, 0}^{q / p} \hat{\mathcal{C}}_{9, q}^{(7)}-4 F_{A}^{q / p} \hat{\mathcal{C}}_{4, q}^{(6)}\right) \\
c_{5}^{p}= & \frac{2 \alpha Q_{p} m_{N}}{\pi \vec{q}^{2}} \hat{\mathcal{C}}_{1}^{(5)}, \\
c_{6}^{p}= & \frac{2 \alpha}{\pi \vec{q}^{2}} \mu_{p} m_{N} \hat{\mathcal{C}}_{1}^{(5)}+\sum_{q}\left(F_{P^{\prime}}^{q / p} \hat{\mathcal{C}}_{4, q}^{(6)}+\frac{m_{N}}{m_{\chi}} F_{P}^{q / p} \hat{\mathcal{C}}_{8, q}^{(7)}\right)+\frac{m_{N}}{m_{\chi}} F_{\tilde{G}}^{p} \hat{\mathcal{C}}_{4}^{(7)}, \\
c_{7}^{p}= & -2 \sum_{q} F_{A}^{q / p} \hat{\mathcal{C}}_{3, q}^{(6)}, \\
c_{8}^{p}= & 2 \sum_{q} F_{1}^{q / p} \hat{\mathcal{C}}_{2, q}^{(6)}, \\
c_{9}^{p}= & 2 \sum_{q}\left[\left(F_{1}^{q / p}+F_{2}^{q / p}\right) \hat{\mathcal{C}}_{2, q}^{(6)}+\frac{m_{N}}{m_{\chi}} F_{A}^{q / p} \hat{\mathcal{C}}_{3, q}^{(7)}\right] \\
c_{10}^{p}= & F_{\tilde{G}}^{p} \hat{\mathcal{C}}_{3}^{(7)}+\sum_{q}\left(F_{P}^{q / p} \hat{\mathcal{C}}_{7, q}^{(7)}-2 \frac{m_{N}}{m_{\chi}} F_{T, 0}^{q / p} \hat{\mathcal{C}}_{10, q}^{(7)}\right), \\
c_{11}^{p}= & \frac{2 \alpha}{\pi} Q_{p} \frac{m_{N}}{\vec{q}^{2}} \hat{\mathcal{C}}_{2}^{(5)}+\sum_{q}\left[2\left(F_{T, 0}^{q / p}-F_{T, 1}^{q / p}\right) \hat{\mathcal{C}}_{10, q}^{(7)}-\frac{m_{N}}{m_{\chi}} F_{S}^{q / p} \hat{\mathcal{C}}_{6, q}^{(7)}\right]-\frac{m_{N}}{m_{\chi}} F_{G}^{p} \hat{\mathcal{C}}_{2}^{(7)}, \\
c_{12}^{p}= & -8 \sum_{q} F_{T, 0}^{q / p} \hat{\mathcal{C}}_{10, q}^{(7)} \cdot \\
&
\end{aligned}
$$

The coefficients for neutrons are obtained by replacing $p \rightarrow n, u \leftrightarrow d$. Above we kept only the chirally leading contributions and listed the results only for the non-vanishing $c_{\mathrm{NR}, i}^{N}$ (i.e., one has $c_{\mathrm{NR}, 2}^{N}=c_{\mathrm{NR}, 3}^{N}=0$ ). For the coefficient $c_{\mathrm{NR}, 1}^{N}$, we also kept the $q^{2}$-suppressed contribution from $\hat{\mathcal{C}}_{9, q},{ }^{(7)}$ that is, however, coherently enhanced. The contributions due to the magnetic and electric dipole operators, eqs. (1.2), are given in appendix A of [1].

In the LO expressions most of the form factors are evaluated at $q^{2}=0$, with the numerical values for $F_{1}^{q / N}$ given in eq. (A.1); for $F_{2}^{q / N}$ in eqs. (A.3)-(A.5); for $F_{A}^{q / N}$ in eq. (A.14) together with eqs. (A.17), (A.19); for $F_{S}^{q / N}$ in eq. (A.27) together with eqs. (A.28), (A.29); for $F_{G}^{q / N}$ in eq. (A.36) together with (A.38); for $F_{T, 0}^{q / N}$ in eq. (A.47) together with (A.48); and for $F_{T, 1}^{q / N}$ in eq. (A.45) together with (A.49)-(A.51). The form factors $F_{P}^{q / N}, F_{P^{\prime}}^{q / N}$, $F_{\tilde{G}}^{q / N}$ contain pion and eta poles. The numerical values for $F_{P^{\prime}}^{q / N}$ are given in eq. (A.13) together with eqs. (A.15), (A.16), (A.18), (A.19); for $F_{P}^{q / N}$ in eq. (A.30)-(A.33) together with eqs. (A.18), (A.19); for $F_{\tilde{G}}^{q / N}$ in eq. (A.42) together with (A.18), (A.19). 
Open Access. This article is distributed under the terms of the Creative Commons Attribution License (CC-BY 4.0), which permits any use, distribution and reproduction in any medium, provided the original author(s) and source are credited.

\section{References}

[1] F. Bishara, J. Brod, B. Grinstein and J. Zupan, Chiral Effective Theory of Dark Matter Direct Detection, JCAP 02 (2017) 009 [arXiv: 1611.00368] [INSPIRE].

[2] J. Fan, M. Reece and L.-T. Wang, Non-relativistic effective theory of dark matter direct detection, JCAP 11 (2010) 042 [arXiv:1008.1591] [INSPIRE].

[3] A.L. Fitzpatrick, W. Haxton, E. Katz, N. Lubbers and Y. Xu, The Effective Field Theory of Dark Matter Direct Detection, JCAP 02 (2013) 004 [arXiv:1203.3542] [INSPIRE].

[4] A.L. Fitzpatrick, W. Haxton, E. Katz, N. Lubbers and Y. Xu, Model Independent Direct Detection Analyses, arXiv:1211.2818 [inSPIRE].

[5] N. Anand, A.L. Fitzpatrick and W.C. Haxton, Weakly interacting massive particle-nucleus elastic scattering response, Phys. Rev. C 89 (2014) 065501 [arXiv:1308.6288] [INSPIRE].

[6] M. Cirelli, E. Del Nobile and P. Panci, Tools for model-independent bounds in direct dark matter searches, JCAP 10 (2013) 019 [arXiv:1307.5955] [INSPIRE].

[7] G. Barello, S. Chang and C.A. Newby, A Model Independent Approach to Inelastic Dark Matter Scattering, Phys. Rev. D 90 (2014) 094027 [arXiv:1409.0536] [INSPIRE].

[8] R.J. Hill and M.P. Solon, Standard Model anatomy of WIMP dark matter direct detection II: QCD analysis and hadronic matrix elements, Phys. Rev. D 91 (2015) 043505 [arXiv: 1409.8290] [INSPIRE].

[9] R. Catena and P. Gondolo, Global fits of the dark matter-nucleon effective interactions, JCAP 09 (2014) 045 [arXiv: 1405.2637] [INSPIRE].

[10] J. Kopp, T. Schwetz and J. Zupan, Global interpretation of direct Dark Matter searches after CDMS-II results, JCAP 02 (2010) 014 [arXiv:0912.4264] [INSPIRE].

[11] R.J. Hill and M.P. Solon, WIMP-nucleon scattering with heavy WIMP effective theory, Phys. Rev. Lett. 112 (2014) 211602 [arXiv:1309.4092] [INSPIRE].

[12] R.J. Hill and M.P. Solon, Universal behavior in the scattering of heavy, weakly interacting dark matter on nuclear targets, Phys. Lett. B 707 (2012) 539 [arXiv:1111.0016] [InSPIRE].

[13] A. Kurylov and M. Kamionkowski, Generalized analysis of weakly interacting massive particle searches, Phys. Rev. D 69 (2004) 063503 [hep-ph/0307185] [INSPIRE].

[14] M. Pospelov and T. ter Veldhuis, Direct and indirect limits on the electromagnetic form-factors of WIMPs, Phys. Lett. B 480 (2000) 181 [hep-ph/0003010] [INSPIRE].

[15] J. Bagnasco, M. Dine and S.D. Thomas, Detecting technibaryon dark matter, Phys. Lett. B 320 (1994) 99 [hep-ph/9310290] [INSPIRE].

[16] V. Cirigliano, M.L. Graesser and G. Ovanesyan, WIMP-nucleus scattering in chiral effective theory, JHEP 10 (2012) 025 [arXiv: 1205. 2695] [INSPIRE].

[17] M. Hoferichter, P. Klos and A. Schwenk, Chiral power counting of one- and two-body currents in direct detection of dark matter, Phys. Lett. B 746 (2015) 410 [arXiv: 1503.04811] [INSPIRE]. 
[18] M. Hoferichter, P. Klos, J. Menéndez and A. Schwenk, Analysis strategies for general spin-independent WIMP-nucleus scattering, Phys. Rev. D 94 (2016) 063505 [arXiv: 1605.08043] [INSPIRE].

[19] R. Catena, A. Ibarra and S. Wild, DAMA confronts null searches in the effective theory of dark matter-nucleon interactions, JCAP 05 (2016) 039 [arXiv: 1602.04074] [INSPIRE].

[20] S. Weinberg, Nuclear forces from chiral Lagrangians, Phys. Lett. B 251 (1990) 288 [INSPIRE].

[21] S. Weinberg, Effective chiral Lagrangians for nucleon-pion interactions and nuclear forces, Nucl. Phys. B 363 (1991) 3 [inSPIRE].

[22] P.F. Bedaque and U. van Kolck, Effective field theory for few nucleon systems, Ann. Rev. Nucl. Part. Sci. 52 (2002) 339 [nucl-th/0203055] [INSPIRE].

[23] E. Epelbaum, H.-W. Hammer and U.-G. Meißner, Modern Theory of Nuclear Forces, Rev. Mod. Phys. 81 (2009) 1773 [arXiv:0811.1338] [InSPIRE].

[24] E. Epelbaum, Nuclear Forces from Chiral Effective Field Theory: A Primer, arXiv:1001.3229 [INSPIRE].

[25] D. Gazda, R. Catena and C. Forssén, Ab initio nuclear response functions for dark matter searches, Phys. Rev. D 95 (2017) 103011 [arXiv:1612.09165] [INSPIRE].

[26] C. Körber, A. Nogga and J. de Vries, First-principle calculations of Dark Matter scattering off light nuclei, Phys. Rev. C 96 (2017) 035805 [arXiv:1704.01150] [INSPIRE].

[27] J. Brod, A. Gootjes-Dreesbach, M. Tammaro and J. Zupan, Effective Field Theory for Dark Matter Direct Detection up to Dimension Seven, arXiv:1710.10218 [INSPIRE].

[28] PICO collaboration, C. Amole et al., Dark Matter Search Results from the PICO-60 $C_{3} F_{8}$ Bubble Chamber, Phys. Rev. Lett. 118 (2017) 251301 [arXiv: 1702.07666] [INSPIRE].

[29] LUX collaboration, D.S. Akerib et al., Limits on spin-dependent WIMP-nucleon cross section obtained from the complete LUX exposure, Phys. Rev. Lett. 118 (2017) 251302 [arXiv: 1705. 03380] [INSPIRE].

[30] J. Goodman, M. Ibe, A. Rajaraman, W. Shepherd, T.M.P. Tait and H.-B. Yu, Constraints on Dark Matter from Colliders, Phys. Rev. D 82 (2010) 116010 [arXiv: 1008.1783] [InSPIRE].

[31] G. Ovanesyan and L. Vecchi, Direct detection of dark matter polarizability, JHEP 07 (2015) 128 [arXiv: 1410.0601] [INSPIRE].

[32] E.E. Jenkins and A.V. Manohar, Baryon chiral perturbation theory using a heavy fermion lagrangian, Phys. Lett. B 255 (1991) 558.

[33] J. Heinonen, R.J. Hill and M.P. Solon, Lorentz invariance in heavy particle effective theories, Phys. Rev. D 86 (2012) 094020 [arXiv: 1208.0601] [INSPIRE].

[34] V. Cirigliano, S. Davidson and Y. Kuno, Spin-dependent $\mu \rightarrow e$ conversion, Phys. Lett. B 771 (2017) 242 [arXiv:1703.02057] [INSPIRE]

[35] Particle Data Group collaboration, C. Patrignani et al., Review of Particle Physics, Chin. Phys. C 40 (2016) 100001 [inSPIRE].

[36] D.J. Gross, S.B. Treiman and F. Wilczek, Light Quark Masses and Isospin Violation, Phys. Rev. D 19 (1979) 2188 [INSPIRE].

[37] XENON collaboration, E. Aprile et al., Effective field theory search for high-energy nuclear recoils using the XENON100 dark matter detector, Phys. Rev. D 96 (2017) 042004 [arXiv: 1705.02614] [INSPIRE]. 
[38] R.S. Sufian, Y.-B. Yang, A. Alexandru, T. Draper, J. Liang and K.-F. Liu, Strange Quark Magnetic Moment of the Nucleon at the Physical Point, Phys. Rev. Lett. 118 (2017) 042001 [arXiv: 1606. 07075] [INSPIRE].

[39] J. Green et al., High-precision calculation of the strange nucleon electromagnetic form factors, Phys. Rev. D 92 (2015) 031501 [arXiv:1505.01803] [INSPIRE].

[40] P.J. Mohr, D.B. Newell and B.N. Taylor, CODATA Recommended Values of the Fundamental Physical Constants: 2014, Rev. Mod. Phys. 88 (2016) 035009 [arXiv:1507.07956] [InSPIRE].

[41] F.J. Ernst, R.G. Sachs and K.C. Wali, Electromagnetic form factors of the nucleon, Phys. Rev. 119 (1960) 1105 [INSPIRE].

[42] R.J. Hill and G. Paz, Model independent extraction of the proton charge radius from electron scattering, Phys. Rev. D 82 (2010) 113005 [arXiv:1008.4619] [InSPIRE].

[43] G. Grilli di Cortona, E. Hardy, J. Pardo Vega and G. Villadoro, The QCD axion, precisely, JHEP 01 (2016) 034 [arXiv: 1511.02867] [InSPIRE].

[44] QCDSF collaboration, G.S. Bali et al., Strangeness Contribution to the Proton Spin from Lattice QCD, Phys. Rev. Lett. 108 (2012) 222001 [arXiv:1112.3354] [INSPIRE].

[45] M. Engelhardt, Strange quark contributions to nucleon mass and spin from lattice QCD, Phys. Rev. D 86 (2012) 114510 [arXiv:1210.0025] [InSPIRE].

[46] T. Bhattacharya, R. Gupta and B. Yoon, Disconnected Quark Loop Contributions to Nucleon Structure, PoS (LATTICE2014) 141 [arXiv: 1503.05975] [INSPIRE].

[47] C. Alexandrou et al., Nucleon axial form factors using $N_{f}=2$ twisted mass fermions with a physical value of the pion mass, Phys. Rev. D 96 (2017) 054507 [arXiv:1705.03399] [INSPIRE].

[48] HERMES collaboration, A. Airapetian et al., Precise determination of the spin structure function $g(1)$ of the proton, deuteron and neutron, Phys. Rev. D 75 (2007) 012007 [hep-ex/0609039] [INSPIRE].

[49] COMPASS collaboration, V. Yu. Alexakhin et al., The Deuteron Spin-dependent Structure Function g1(d) and its First Moment, Phys. Lett. B 647 (2007) 8 [hep-ex/0609038] [INSPIRE].

[50] A.S. Meyer, M. Betancourt, R. Gran and R.J. Hill, Deuterium target data for precision neutrino-nucleus cross sections, Phys. Rev. D 93 (2016) 113015 [arXiv:1603.03048] [INSPIRE].

[51] V. Bernard, L. Elouadrhiri and U.-G. Meißner, Axial structure of the nucleon: Topical Review, J. Phys. G 28 (2002) R1 [hep-ph/0107088] [inSPIRE].

[52] MiniBoonE collaboration, A.A. Aguilar-Arevalo et al., First Measurement of the Muon Neutrino Charged Current Quasielastic Double Differential Cross Section, Phys. Rev. D 81 (2010) 092005 [arXiv: 1002.2680] [INSPIRE].

[53] G. Rajan, J. Yong-Chull, L. Huey-Wen, Y. Boram and B. Tanmoy, Axial Vector Form Factors of the Nucleon from Lattice QCD, arXiv:1705.06834 [INSPIRE].

[54] LHPC collaboration, J.D. Bratt et al., Nucleon structure from mixed action calculations using $2+1$ flavors of asqtad sea and domain wall valence fermions, Phys. Rev. D 82 (2010) 094502 [arXiv: 1001.3620] [inSPIRE].

[55] V. Bernard, H.W. Fearing, T.R. Hemmert and U.G. Meißner, The form-factors of the nucleon at small momentum transfer, Nucl. Phys. A 635 (1998) 121 [Erratum ibid. A 642 (1998) 563] [hep-ph/9801297] [INSPIRE]. 
[56] P. Junnarkar and A. Walker-Loud, Scalar strange content of the nucleon from lattice QCD, Phys. Rev. D 87 (2013) 114510 [arXiv:1301.1114] [InSPIRE].

[57] XQCD collaboration, Y.-B. Yang, A. Alexandru, T. Draper, J. Liang and K.-F. Liu, $\pi N$ and strangeness sigma terms at the physical point with chiral fermions, Phys. Rev. D 94 (2016) 054503 [arXiv: 1511.09089] [INSPIRE].

[58] S. Dürr et al., Lattice computation of the nucleon scalar quark contents at the physical point, Phys. Rev. Lett. 116 (2016) 172001 [arXiv:1510.08013] [INSPIRE].

[59] J.M. Alarcon, J. Martin Camalich and J.A. Oller, The chiral representation of the $\pi N$ scattering amplitude and the pion-nucleon sigma term, Phys. Rev. D 85 (2012) 051503 [arXiv:1110.3797] [INSPIRE].

[60] J. Ruiz de Elvira, M. Hoferichter, B. Kubis and U.-G. Meißner, Extracting the sigma-term from low-energy pion-nucleon scattering, arXiv:1706.01465 [INSPIRE].

[61] M. Hoferichter, J. Ruiz de Elvira, B. Kubis and U.-G. Meißner, High-Precision Determination of the Pion-Nucleon $\sigma$-term from Roy-Steiner Equations, Phys. Rev. Lett. 115 (2015) 092301 [arXiv: 1506.04142] [INSPIRE].

[62] L. Álvarez-Ruso, T. Ledwig, J. Martin Camalich and M.J. Vicente Vacas, Nucleon mass and pion-nucleon sigma term from a chiral analysis of lattice QCD world data, EPJ Web Conf. 73 (2014) 04015 [INSPIRE].

[63] ETM collaboration, A. Abdel-Rehim et al., Direct Evaluation of the Quark Content of Nucleons from Lattice QCD at the Physical Point, Phys. Rev. Lett. 116 (2016) 252001 [arXiv: 1601.01624] [INSPIRE].

[64] M. Hoferichter, J. Ruiz de Elvira, B. Kubis and U.-G. Meißner, Remarks on the pion-nucleon $\sigma$-term, Phys. Lett. B 760 (2016) 74 [arXiv:1602.07688] [INSPIRE].

[65] A. Crivellin, M. Hoferichter and M. Procura, Accurate evaluation of hadronic uncertainties in spin-independent WIMP-nucleon scattering: Disentangling two- and three-flavor effects, Phys. Rev. D 89 (2014) 054021 [arXiv: 1312.4951] [INSPIRE].

[66] C. McNeile et al., Direct determination of the strange and light quark condensates from full lattice QCD, Phys. Rev. D 87 (2013) 034503 [arXiv:1211.6577] [InSPIRE].

[67] UKQCD, QCDSF collaboration, M. Gockeler et al., Quark helicity flip generalized parton distributions from two-flavor lattice QCD, Phys. Lett. B 627 (2005) 113 [hep-lat/0507001] [INSPIRE].

[68] M. Diehl, Generalized parton distributions with helicity flip, Eur. Phys. J. C 19 (2001) 485 [hep-ph/0101335] [INSPIRE].

[69] C. Alexandrou et al., Nucleon scalar and tensor charges using lattice QCD simulations at the physical value of the pion mass, Phys. Rev. D 95 (2017) 114514 [arXiv:1703.08788] [INSPIRE].

[70] T. Bhattacharya, V. Cirigliano, S. Cohen, R. Gupta, H.-W. Lin and B. Yoon, Axial, Scalar and Tensor Charges of the Nucleon from $2+1+1$-flavor Lattice QCD, Phys. Rev. D 94 (2016) 054508 [arXiv: 1606.07049] [INSPIRE].

[71] QCDSF/UKQCD collaboration, D. Pleiter et al., Nucleon form factors and structure functions from $N_{f}=2$ Clover fermions, PoS(LATTICE 2010) 153 [arXiv:1101.2326] [INSPIRE]. 
[72] G.S. Bali et al., Nucleon isovector couplings from $N_{f}=2$ lattice QCD, Phys. Rev. D 91 (2015) 054501 [arXiv: 1412.7336] [INSPIRE].

[73] PNDME collaboration, T. Bhattacharya et al., Iso-vector and Iso-scalar Tensor Charges of the Nucleon from Lattice QCD, Phys. Rev. D 92 (2015) 094511 [arXiv:1506.06411] [INSPIRE].

[74] H.-W. Lin, T. Blum, S. Ohta, S. Sasaki and T. Yamazaki, Nucleon structure with two flavors of dynamical domain-wall fermions, Phys. Rev. D 78 (2008) 014505 [arXiv:0802.0863] [INSPIRE].

[75] J.R. Green, J.W. Negele, A.V. Pochinsky, S.N. Syritsyn, M. Engelhardt and S. Krieg, Nucleon Scalar and Tensor Charges from Lattice QCD with Light Wilson Quarks, Phys. Rev. D 86 (2012) 114509 [arXiv: 1206.4527] [INSPIRE].

[76] C. Alexandrou, M. Constantinou, K. Jansen, G. Koutsou and H. Panagopoulos, Nucleon transversity generalized form factors with twisted mass fermions, PoS (LATTICE 2013) 294 [arXiv: 1311.4670] [INSPIRE].

[77] A. Abdel-Rehim et al., Nucleon and pion structure with lattice QCD simulations at physical value of the pion mass, Phys. Rev. D 92 (2015) 114513 [arXiv:1507.04936] [INSPIRE].

[78] B. Pasquini, M. Pincetti and S. Boffi, Chiral-odd generalized parton distributions in constituent quark models, Phys. Rev. D 72 (2005) 094029 [hep-ph/0510376] [INSPIRE].

[79] H. Gao, T. Liu and Z. Zhao, Nucleon tensor charge and electric dipole moment, arXiv:1704.00113 [INSPIRE].

[80] UKQCD, QCDSF collaboration, M. Gockeler et al., Transverse spin structure of the nucleon from lattice QCD simulations, Phys. Rev. Lett. 98 (2007) 222001 [hep-lat/0612032] [INSPIRE].

[81] I. Schmidt and J. Soffer, Melosh rotation and the nucleon tensor charge, Phys. Lett. B 407 (1997) 331 [hep-ph/9703411] [INSPIRE].

[82] T. Ledwig, A. Silva and H.-C. Kim, Anomalous tensor magnetic moments and form factors of the proton in the self-consistent chiral quark-soliton model, Phys. Rev. D 82 (2010) 054014 [arXiv: 1007.1355 ] [INSPIRE].

[83] T. Ledwig and H.-C. Kim, Transverse strange quark spin structure of the nucleon, Phys. Rev. D 85 (2012) 034041 [arXiv:1107.4952] [INSPIRE].

[84] J. Zanotti et al., Transverse spin densities of octet baryons using Lattice QCD, PoS (LATTICE2016) 163.

[85] A.F. Falk, M.E. Luke and M.J. Savage, Nonperturbative contributions to the inclusive rare decays $B \rightarrow X_{s} \gamma$ and $B \rightarrow X_{s} l^{+} l^{-}$, Phys. Rev. D 49 (1994) 3367 [hep-ph/9308288] [INSPIRE].

[86] A.V. Manohar, The HQET/NRQCD Lagrangian to order $\alpha / m-3$, Phys. Rev. D 56 (1997) 230 [hep-ph/9701294] [INSPIRE].

[87] M.E. Luke and A.V. Manohar, Reparametrization invariance constraints on heavy particle effective field theories, Phys. Lett. B 286 (1992) 348 [hep-ph/9205228] [INSPIRE]. 